Mon. Not. R. Astron. Soc. 000, 1 58 (2015) Printed 21 March $2022 \quad$ (MN LATEX style file v2.2)

\title{
The Way We Measure: Comparison of Methods to Derive Radial Surface Brightness Profiles
}

\author{
S. P. C. Peters ${ }^{1}$, P. C. van der Kruit ${ }^{1 \star}$, R. S. de Jong ${ }^{2}$ \\ ${ }^{1}$ Kapteyn Astronomical Institute, University of Groningen, P.O.Box 800, 9700AV Groningen, the Netherlands \\ ${ }^{2}$ Leibniz Institut für Astrophysik Potsdam (AIP), An der Sternwarte 16, 14482 Potsdam, Germany.
}

Accepted 2015 month xx. Received 2015 Month xx; in original form 2015 Month xx

\begin{abstract}
The breaks and truncations in the luminosity profile of face-on spiral galaxies offer valuable insights in their formation history. The traditional method of deriving the surface photometry profile for face-on galaxies is to use elliptical averaging. In this paper, we explore the question whether elliptical averaging is the best way to do this. We apply two additional surface photometry methods, one new: principle axis summation, and one old that has become seldom used: equivalent profiles. These are compared to elliptically averaged profiles using a set of 29 face-on galaxies. We find that the equivalent profiles match extremely well with elliptically averaged profiles, confirming the validity of using elliptical averaging. The principle axis summation offers a better comparison to edge-on galaxies.
\end{abstract}

Key words: galaxies: photometry, galaxies: spiral, galaxies: structure

\section{INTRODUCTION}

The surface photometry of a galaxy is the relationship of the radius $R$, seen from the centre of a galaxy, with the surface brightness $\mu(R)$. To first order, light is tracing mass in a galaxy. It is therefore an interesting tool for the study of galaxy dynamics and evolution. The first studies on the subject are by Patterson (1940) and de Vaucouleurs (1948. 1959), who noted that the surface brightness of the disc of spiral galaxies followed an exponential decline. The exponential nature was studied in more detail by Freeman (1970), who found that there was a second type of profiles that exhibits a break, beyond which the brightness decreases more rapidly.

The lines-of-sight in an edge-on galaxy are typically longer than in a face-on galaxy. Thus, more stars are sampled by a single line-of-sight through an edge-on than through a face-on galaxy at that same (projected) radius. Because of this, it is easier to detect light at larger radii in edge-on galaxies than in face-on galaxies. This allowed van der Kruit (1979) to note that in three edge-on galaxies, the radius of the stellar disc did not increase with deeper photograp000hic exposures. This work was later expanded to a set of eight edge-on galaxies for which the three-dimensional light distribution was studied in detail. Each of these galaxies has a truncated disc, beyond which the intensity rapidly

* For more information, please contact P.C. van der Kruit by email at vdkruit@astro.rug.nl.. drops to zero, on average after $4.2 \pm 0.6$ radial scale lengths (van der Kruit \& Searle 1981ab, 1982a b). The presence of truncations was confirmed by Pohlen et al. (2000), who found however a ratio of trunction radius to exponential scale length of only $2.9 \pm 0.7$.

Truncations in face-on galaxies have, at least in our view, not been unambiguously identified. Pohlen \& Trujillo (2006) used the Sloan Digital Sky Survey (SDSS) to study a set of 90 face-on late-type galaxies. Pohlen \& Trujillo (2006) identified 14 face-on galaxies with truncations. This result has been disputed by van der Kruit (2008), who argued that these are in fact breaks similar to those found by Freeman (1970). Erwin et al. (2008) studied 66 barred, earlytype galaxies and Gutiérrez et al. (2011) another sample of 47 early-type non-barred spirals. Many of these inclined systems are classified as having 'truncations' (increasingly among later types), but we remain unconvinced that these are equivalent to those in edge-ons and not breaks at higher surface brightness levels. Combining Spitzer and near-IR observations seems to indicate that the break radii correlate with those of rings, lenses or spiral arms, and not with a sharp outer decline (Laine et al. 2014). Bakos et al. (2008) found from a study of radial colour profiles that breaks in the light profiles often do not correspond to breaks in the apparent total stellar mass surface density, in fact leaving no feature whatsoever. Recently Herrmann et al. (2013. 2016) have initiated studiss of a large sample of dwarf galaxies; they find many cases of breaks that (unlike spirals) remain in stellar surface density profiles. Exponential gas disks can 
have a double exponential star formation rate, the break radius being related to the instability (Elmegreen \& Hunter 2006). Comerón et al. (2012) studied 70 edge-on galaxies from the Spitzer Survey of Stellar Structure in Galaxies $\left(S^{4} \mathrm{G}\right)$ and found that many edge-ons have truncations, while often more inward breaks could be identified, that occured at similar positions as those measured in face-on galaxies by Pohlen \& Trujillo (2006).

The view of breaks and truncations as two separate features was put forward by Martín-Navarro et al. (2012). In a study of 34 highly inclined spiral galaxies, they found that the innermost break occurs at $\sim 8 \pm 1 \mathrm{kpc}$ and truncations at $\sim 14 \pm 2 \mathrm{kpc}$ in galaxies. It should be stated that not all workers agree with this point of view. In particular Erwin et al. (2008), but also Erwin et al. (2005) and Pohlen \& Trujillo (2006), argue that the breaks really correspond to the truncations in edge-on galaxies. We disagree, but will return to this subject more extensively in the next paper in our studies (Peters et al. 2015).

Anti-truncated profiles, in which the intensity drops less quickly beyond the break than it did before the break, have also been discovered (Erwin et al. 2005). We no further address this issue in this paper, but will discuss it in more detail in the next paper (Peters et al. 2015).

Part of the problem in detecting truncations originates in the different ways profiles from edge-ons and face-ons are extracted. In edge-on galaxies, the surface photometry is defined as the surface brightness along the major axis of the galaxy. This light comes from a variety of radii as the lineof-sight crosses through the galaxy. In face-on galaxies, the most common way to derive profiles is by performing elliptical averaging, such as that offered by the IRAF package ellipse (Jedrzejewski 1987, Busko 1996). Light in such a profile only comes from structures at a single radius. The averaging cancels out any local structure, which might be causing the truncations in edge-ons van der Kruit \& Freeman 2011).

We believe that these local structures are of importance when looking for disc truncations. It is therefore interesting to see what the impact of ellipse averaging is on profiles, and to explore alternative ways to derive such profiles. We use two different methods for deriving surface brightness profiles in face-on galaxies that should be less sensitive to local structure and deviations from circular symmetry: the Principle Axis Summation and the Equivalent Profiles. In Section 2, we will detail the inner workings of these methods. We will present our sample of face-on galaxies, based on a sub-sample of the work by Pohlen \& Trujillo (2006), in Section 3. In Section 4, the data will be analyzed and discussed, followed by the conclusions in Section 5. In order to conserve trees, the online Appendix contains tables and figures for individual galaxies.

\section{SURFACE PHOTOMETRY METHODS}

\subsection{Principle Axis Summation}

The active debate over the nature of truncations in edge-ons versus face-ons sparked our interest in developing a new way of measuring the profiles. While attempts have been made to decompose edge-on galaxies into face-ons, such as van der

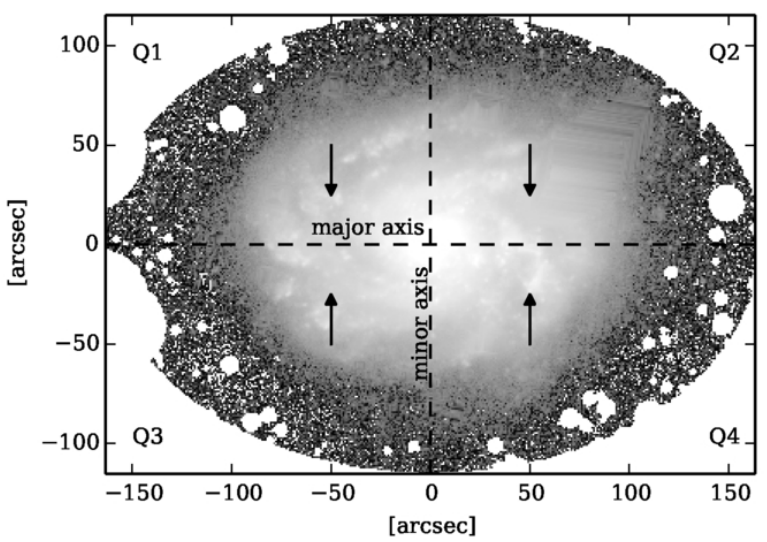

Figure 1. Demonstration of the terminology used in the PAS method. The shown galaxy is NGC 450. The major and minor axes are shown using the dashed lines. Each quadrant has been labelled. The direction in which the data is summed is shown using the arrows. The outlines of the mask covering background galaxy UGC 807 are visible in quadrant Q2. This quadrant is therefore ignored in the final PAS analysis.

Kruit \& Searle (1981a), Pohlen et al. (2007), Pohlen et al. (2004), Pohlen et al. (2007) and Comerón et al. (2012), no real attempt has been made to project face-ons into edgeons. This enticed us to develop this first method, the Principle Axis Summation (PAS). While not a true projection into an edge-on geometry, the PAS results resemble the edge-on geometry more closely than those using ellipse-fit profiles.

The PAS method partitions the face-on galaxy into four quadrants, centred on its major and minor axis. Each quadrant is summed onto the major axis, leaving four quadrantprofiles $Q_{1}(R), Q_{2}(R), Q_{3}(R)$ and $Q_{4}(R)$ (see Figure 11). These are multiplied by two, to represent the full line-ofsight along the major axis and to represent the line-of-sight integration in an edge-on galaxy better. The main profile $P(R)$ is taken as the median of these four. The scatter between the four quadrants is a good measure of any asymmetry in the galaxy. In cases where one or more quadrants suffer from severe contamination by foreground or background objects, that quadrant can be ignored and only the clean quadrants will be used for the median. A clear example of this is in NGC 450, where background galaxy UGC 807 is covering a significant part of a quadrant (Quadrant Q2 in Figure 1). We run a dynamic binning algorithm along the main profile, to ensure that each point has at least a signal-to-noise ratio of two. We use an elliptical blanking mask around each galaxy, shaped and oriented according to the 25th magnitude ellipse of the galaxy and blanked beyond a trust radius $R_{\mathrm{t}}$, to minimize the contribution of sky noise. The trust radius $R_{\mathrm{t}}$ is determined by eye on a heavily smoothed image, such that the galaxy is fully included in the mask.

The noise in each quadrants profile is a combination of the intrinsic pixel-to-pixel noise, any large-scale fluctuations and blanked regions. It thus varies with radius as the amount of pixels in the summation changes. The main profile depends on the combination of four of these varying 

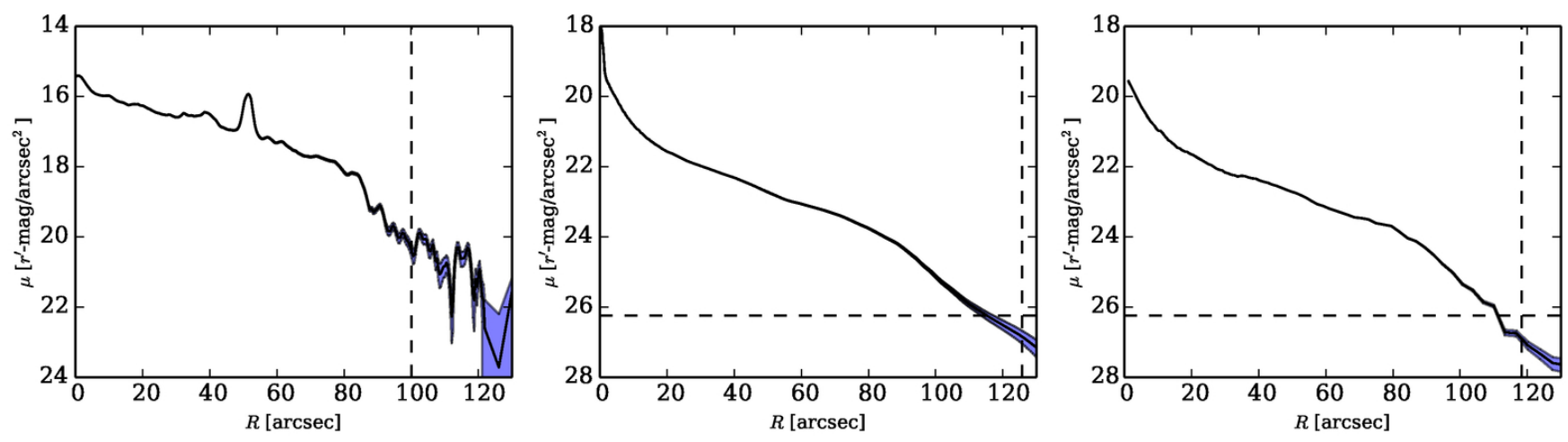

Figure 2. Demonstration of the sensitivity to background under- and overestimation. Here we have used an offset of 0.1 ADU. The horizontal, dashed line shows the level of the pixel-to-pixel noise. The vertical, dashed line shows the point where the profile deviate by more than 0.2 magnitudes, which represents the point up to which we trust the profile. From left to right the panels correspond to the PAS, EP and ellipse-fit methods. The profiles are based on NGC 450 (see Figure A4.

quadrants and can thus vary drastically. To have a good representation of the noise levels we calculate the noise in the profiles using the sky, as taken from the ellipse between one and two times the trust radius $R_{\mathrm{t}}$. All pixels between these two radii are selected and merged row by row into a single long row of pixels. For each quadrant, we smooth a copy of this row of sky pixels with a 'tophat' kernel with the length of the amount of pixels used, effectively recreating the pixel summation. We randomly select a value out of each of these four smoothed sets and take the median. This is repeated 10000 times and the noise is then calculated as the standard deviation of this sample.

There are two major differences between this projection and a true edge-on. First, in real edge-ons we would be able to observe the effect of variations with height. As we are seeing the galaxy from above, PAS cannot show this effect. Compared to ellipse averaging, due to the summation the surface brightness in PAS will also be brighter. The summation effectively has the unit magnitude per arcsec, making it distance dependent. For a true comparison with edge-ons, one would therefore need to apply the PAS method to those as well. The overall shape of the profile should however be equal. A second major difference is that dust absorption is less of an issue here. In a true edge-on, this could have a significant impact on the scale length of the profile. This is an interesting feature, as a statistical comparison in a large sample of edge-ons and face-ons could be used to analyze the dust content of galaxies.

The PAS profiles are more susceptible to sky determination issues than ellipse-fit profiles, as any remaining background offset will be multiplied by the amount of pixels along the minor axis instead of being averaged. In this paper, we will use the uncertainty in the background-offset estimation (see Section 3.6 to over- and under-subtract the profile. We place our confidence limit at the spot where these three profiles start to deviate by more than 0.2 magnitudes. In Figure 2 (left), the sensitivity to the background is demonstrated, by over- and under-subtracting the data by 0.1 ADU.

As noted before, the PAS profiles are effectively in units of magnitude per arcsec. Because of this, direct comparison with the other two types of profiles is hard. Still, we have chosen to display all these profiles together in one graph, by applying an offset to the PAS profiles, such that at $R=0$ the brightest EP profile begins at the same magnitude as the faintest PAS profile. Direct comparisons of the brightness of the PAS profile with the EP and ellipse-fit profiles should not be made. This strategy does however allow for the check if a feature occurs at a particular radius $R$ in all three types of profiles.

\subsection{The Equivalent Profiles}

The Equivalent Profiles (EP) are a radical twist on the usual methods. Instead of using the radius $R$ to find the surface brightness $\mu(R)$ in a face-on galaxy, the method turns things around. For each observed surface brightness $\mu$ in the image, there will always be some number of pixels $N(\mu)$ that have that or a brighter value. Since each pixel covers a small surface $d A$, a total equivalent surface $A(\mu)$ can be formed. In SDSS, the area of each pixel covers $0.396 \times 0.396 \operatorname{arcsec}^{2}$. Assume that the surface brightness in a galaxy is always brightest in the centre and decreases with radius 1 . The surface will then form an ellipse, or circle in the case of a perfect face-on, centred on the galaxy. The radius of this equivalent surface is called the equivalent radius $R(\mu)$. Mathematically we can describe this as

$$
R(\mu)=\sqrt{\frac{N(\mu) d A}{\pi \cos i}},
$$

where $i$ is the inclination of the galaxy.

As an example, suppose for a perfectly face-on galaxy that the brightest pixel in the observation has a value of $\mu=18 r^{\prime}-\mathrm{mag} / \operatorname{arcsec}^{2}$. Since this value is only reached in one pixel, the equivalent area $A(\mu)$ is only $0.396^{2} \operatorname{arcsec}^{2}$, and the equivalent radius $R(\mu)$ is thus 0.35 arcsec. At $\mu=20$ $r$ '-mag/ $\operatorname{arcsec}^{2}$ there could be 10.000 pixels at that or a brighter value. In that case the equivalent area $A(\mu)$ goes up to $0.396^{2} \times 10.000=1568 \operatorname{arcsec}^{2}$, and the equivalent radius $R(\mu)$ is thus 22.3". By repeating this process for every value of $\mu$ in the observation, we can thus build up the associated set of equivalent radii.

1 With the exception of small-scale features, this holds for all three types of profiles, the only differences between them is the rate at which the brightness decreases. 
Tests show that this method is particularly sensitive to background noise. Any positive component of the noise distribution will add to surface $A(\mu)$ and thus increase radius $R(\mu)$. This creates a drastic increase in the equivalent radius at the faintest surface brightness levels (see for example Figure 3. The other methods suffer much less from this, as the positive noise values are averaged out against the negative noise values. Two techniques are used to deal with this. Firstly, similar as in the PAS, we use an elliptical blanking mask around the galaxy. It is centred on the galaxy and has sufficient radius not to blank the galaxy itself, but leaves as little background as possible. This blocks out all signals for which we are sure that they are unrelated to the galaxy. Secondly, we use non-linear anisotropic filtering, an algorithm normally used in magnetic resonance imaging (Jones et al. 2003). This helps smooth low $\mathrm{S} / \mathrm{N}$ regions, while conserving the flux and important structure in the image.

Equivalent Profiles are an old method, going back more than 60 years. The oldest reference traces back to de Vaucouleurs (1948), wherein he derives his famous $R^{1 / 4}$ profile. In the decades beyond, they were used quite often, as for example in the photometric survey by van der Kruit (1979). The newer elliptically averaged profiles suffer less from noise, and are able to vary the position angle and inclination as function of radius (Jedrzejewski 1987), things that the Equivalent Profiles cannot. This is likely why the Equivalent Profiles have fallen from grace.

Similar to the PAS, the confidence limit of the profile is again calculated by over- and under-subtracting the data by two times the uncertainty and establishing where the profiles start to deviate by more than 0.2 magnitudes. We demonstrate this contamination by background noise in Figure 2 (middle). Comparing it to the profiles from the elliptical averaging (reproduced here from Pohlen \& Trujillo (2006)); we see that the Equivalent Profiles start to suffer at a brighter magnitude levels. In practice, this level is slightly higher than the background pixel-to-pixel noise $\sigma$. The choice of the radius of the mask is also not trivial, as demonstrated in Figure 3 The larger the radius, the more background is sampled, and the more noise is picked up. We have opted to use the same ellipse, with trust radius $R_{\mathrm{t}}$ as used for the PAS.

\section{DATA}

\subsection{Sample}

We use the full sample defined by Pohlen \& Trujillo (2006). They used the following criteria to define their sample:

- A Hubble type $T$ parameter between $2.99<T<8.49$. This corresponds to an intermediate to late-type galaxy sample with $\mathrm{Sb}$ to $\mathrm{Sdm}$ galaxies.

- The axis ratio is chosen such that the inclination is $i<61^{\circ}$, as to avoid the influence of dust and as a convenient way to classify morphological properties of the galaxy that would have been more obscured at higher inclinations.

- The recession velocity is $v_{\mathrm{vir}}<3250 \mathrm{~km} / \mathrm{s}$ and the total B-band brightness $\mathrm{M}_{\mathrm{B} \text {,abs }}<-18.5 \mathrm{~B}-\mathrm{mag}$, as to get a complete sample of galaxies within the $46 \mathrm{Mpc}$ survey distance.

- Galactic latitude $\left\|b_{I I}\right\|>20^{\circ}$ as to avoid dust obscuration.

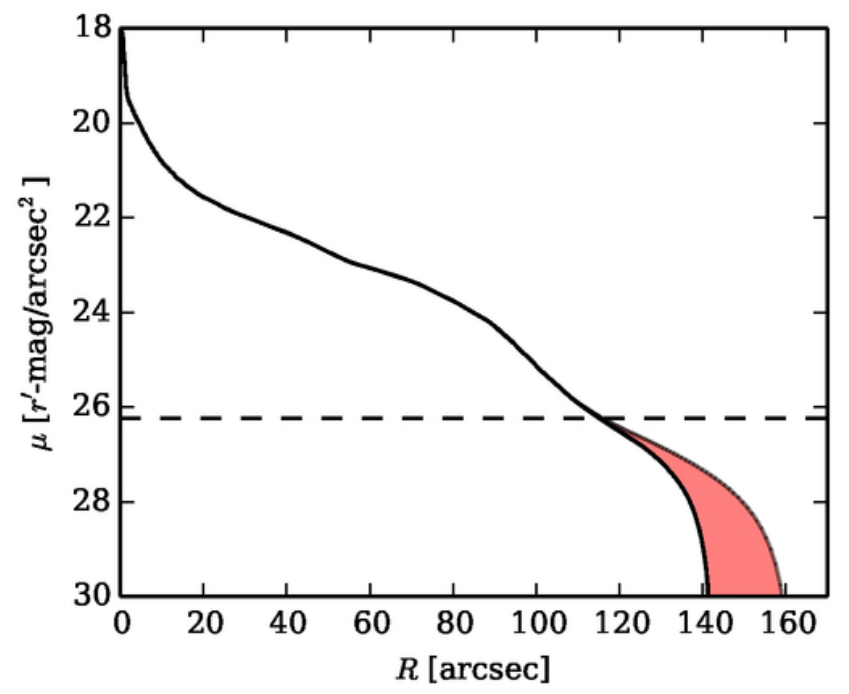

Figure 3. Effect of choice of radius for the elliptical mask on the Equivalent Profiles. The radius of the mask has been increased by $25 \%$. The shaded region shows the increase in profile compared to the original, black profile. The horizontal, dashed line shows the level of the pixel-to-pixel noise.

Using DR2 of SDSS (Abazajian et al. 2004), this led them to a sample of 98 face-on galaxies for which observations were available, out of a full sample of 655 galaxies. The final sample is listed in Table 3.1

\subsection{Data Reduction}

Originally, we retrieved the SDSS images straight from the SDSS website at www.sdss.org. These came from Data Release 7 (Abazajian et al. 2009). Most of the galaxies are so large, however, that their outskirts are often not covered by the frame and mosaicking would be required. Instead of manually mosaicking these images, we opted for a different approach. We used MonTAGE ${ }^{2}$ (Jacob et al. 2010), for the retrieval and mosaicking. In this paper we focus on the $g^{\prime}$, $r^{\prime}$ and $i^{\prime}$ band images.

The following steps were undertaken. The reference header of the final image was created using mHdr. Tasks mArchiveList and mArchiveExec were then used in sequence to retrieve the $g^{\prime}, r^{\prime}$ and $i^{\prime}$ images from SDSS. The images were then projected to the reference frame using mProjExec. The overlaps regions between the images were calculated and extracted, using mOverlaps and mDiffExec. With mFitExec the plane fitting coefficients were calculated between all frames. A model of the background was then created using mBgModel. We did allow it to fit the slope and set the maximum number of iterations to 5 . We correct all frames to the common background using mBgExec. Finally, the images were joined using mAdd.

Each galaxy is thus composed of a set of SDSS frames, which all have a background plane subtracted. We have also run a test wherein only a constant offset correction was performed between frames, but in almost all cases, the planecorrected images were superior to the constant offset cor-

\footnotetext{
2 Montage is available at montage.ipac.caltech.edu/.
} 


\begin{tabular}{|c|c|c|c|c|c|c|c|}
\hline Galaxy & $M_{\mathbf{B}, \mathrm{abs}}$ & Туре & $\mathbf{t}$ & $v_{\text {rot }}[\mathrm{km} / \mathrm{s}]$ & $i\left[^{\circ}\right]$ & $\mathbf{P A}\left[^{\circ}\right]$ & $\mathrm{D}[\mathrm{Mpc}]$ \\
\hline IC1067 & -18.65 & $\mathrm{Sb}$ & 3.0 & 148.74 & 42.3 & 151.1 & 28.3 \\
\hline IC1125 & -20.03 & SBcd & 7.3 & 103.77 & 55.9 & 305.8 & 35 \\
\hline IC1158 & -19.52 & $\mathrm{SABc}$ & 5.1 & 120 & 55.9 & 136.7 & 29.7 \\
\hline NGC0450 & -19.72 & $\mathrm{SABc}$ & 5.8 & 102.94 & 50.2 & 188.8 & 19 \\
\hline NGC0701 & -19.84 & $\mathrm{SBc}$ & 5.0 & 120.96 & 59.3 & 45.7 & 19.5 \\
\hline NGC0853 & -16.23 & $\mathrm{Sm}$ & 8.6 & 60.29 & 50.2 & 16.3 & 21 \\
\hline NGC0941 & -19.13 & $\mathrm{SABc}$ & 5.3 & 88.93 & 45.6 & 101.4 & 22 \\
\hline NGC1042 & -20.27 & $\mathrm{SABc}$ & 6.0 & 46.1 & 36.9 & 74.7 & 4.21 \\
\hline NGC1068 & -21.5 & $\mathrm{Sb}$ & 3.0 & 282.54 & 24.5 & 170.2 & 10.1 \\
\hline NGC1084 & -20.63 & $\mathrm{Sc}$ & 4.9 & 194.52 & 52.4 & 52.8 & 16.6 \\
\hline NGC1087 & -20.65 & $\mathrm{SABc}$ & 5.2 & 120.27 & 52.4 & 268.4 & 19 \\
\hline NGC1299 & -19.35 & $\mathrm{SBb}$ & 3.0 & 120.91 & 56.6 & 42.0 & 32 \\
\hline NGC2541 & -18.66 & $\mathrm{SABc}$ & 6.0 & 97.22 & 61.3 & 107.6 & 14.8 \\
\hline NGC2543 & -20.5 & $\mathrm{Sb}$ & 3.0 & 148.11 & 60.0 & 51.1 & 26.3 \\
\hline NGC2684 & -19.88 & $\mathrm{Sc}$ & 4.6 & 101.03 & 34.9 & 35.1 & 44.9 \\
\hline NGC2701 & -20.45 & $\mathrm{SABc}$ & 5.2 & 143.88 & 47.2 & 63.3 & 30.7 \\
\hline NGC2776 & -21.54 & $\mathrm{SABc}$ & 5.2 & 99.06 & 18.2 & 6.0 & 38.7 \\
\hline NGC2967 & -20.37 & $\mathrm{Sc}$ & 5.2 & 165.95 & 21.6 & 250.7 & 30.9 \\
\hline NGC3055 & -20.12 & $\mathrm{SABc}$ & 5.3 & 142.65 & 54.5 & 27.0 & 28 \\
\hline NGC3246 & -19.3 & $\mathrm{Sd}$ & 7.9 & 109.85 & 58.7 & 354.4 & 35.5 \\
\hline NGC3259 & -19.62 & $\mathrm{SABb}$ & 3.7 & 120.54 & 55.2 & 71.5 & 35.9 \\
\hline NGC3310 & -20.11 & $\mathrm{SABb}$ & 4.0 & 288.38 & 18.2 & 70.7 & 17.5 \\
\hline NGC3359 & -20.57 & $\mathrm{Sc}$ & 5.2 & 148.06 & 58.7 & 101.9 & 22.6 \\
\hline NGC3423 & -19.6 & $\mathrm{Sc}$ & 6.0 & 127.12 & 35.9 & 56.3 & 11.7 \\
\hline NGC3488 & -19.9 & $\mathrm{SBc}$ & 5.2 & 122.69 & 48.7 & 92.7 & 46.3 \\
\hline NGC3583 & -20.58 & $\mathrm{SBb}$ & 3.1 & 182.1 & 47.2 & 326.4 & 31.6 \\
\hline NGC3589 & -18.63 & $\mathrm{SABc}$ & 7.0 & 77.82 & 60.0 & 36.3 & 34.1 \\
\hline NGC3631 & -21.02 & $\mathrm{Sc}$ & 5.2 & 78.36 & 32.9 & 339.9 & 21.6 \\
\hline NGC3642 & -20.57 & $\mathrm{Sbc}$ & 4.0 & 48.71 & 18.2 & 7.4 & 27.5 \\
\hline NGC3756 & -20.2 & $\mathrm{SABb}$ & 4.0 & 145.95 & 60.0 & 91.0 & 15.7 \\
\hline NGC3888 & -20.47 & $\mathrm{SABc}$ & 5.3 & 203.06 & 42.3 & 335.8 & 41.5 \\
\hline NGC3893 & -21 & $\mathrm{SABc}$ & 5.2 & 147.68 & 53.8 & 282.4 & 15.7 \\
\hline NGC3982 & -19.91 & $\mathrm{SABb}$ & 3.2 & 191.83 & 27.1 & 92.2 & 24.6 \\
\hline NGC3992 & -21.31 & $\mathrm{Sbc}$ & 4.0 & 295.12 & 54.5 & 19.8 & 22.9 \\
\hline NGC4030 & -20.84 & Sbc & 4.0 & 201.32 & 36.9 & 59.6 & 25 \\
\hline NGC4041 & -20.19 & $\mathrm{Sbc}$ & 4.0 & 263.1 & 18.2 & 12.4 & 22.7 \\
\hline NGC4102 & -19.4 & $\mathrm{SABb}$ & 3.1 & 158.14 & 55.2 & 50.6 & 16 \\
\hline NGC4108 & -20.25 & $\mathrm{Sc}$ & 5.2 & 223.28 & 39.6 & 323.1 & 41.6 \\
\hline NGC4108B & -18.77 & $\mathrm{SABc}$ & 7.0 & 195.8 & 38.7 & 349.7 & 43.8 \\
\hline NGC4123 & -19.91 & $\mathrm{Sc}$ & 5.0 & 128.5 & 47.9 & 324.0 & 14.9 \\
\hline NGC4210 & -19.99 & $\mathrm{Sb}$ & 3.0 & 162.96 & 40.5 & 351.9 & 44.8 \\
\hline NGC4273 & -20.6 & $\mathrm{Sc}$ & 5.2 & 328.91 & 52.4 & 263.3 & 28.5 \\
\hline NGC4480 & -20.3 & $\mathrm{SABc}$ & 5.1 & 169.24 & 60.0 & 92.6 & 36.7 \\
\hline NGC4517A & -19.8 & $\mathrm{Sd}$ & 7.8 & 71.35 & 55.2 & 241.4 & 23.6 \\
\hline NGC4545 & -20.3 & $\mathrm{Sc}$ & 5.6 & 129.19 & 54.5 & 264.7 & 38.2 \\
\hline NGC4653 & -20.33 & $\mathrm{SABc}$ & 6.0 & 211.75 & 33.9 & 101.5 & 39.1 \\
\hline NGC4668 & -18.92 & SBcd & 7.4 & 62.33 & 58.7 & 265.5 & 17.2 \\
\hline NGC4904 & -19.12 & $\mathrm{Sc}$ & 5.8 & 105.15 & 44.8 & 243.4 & 20.5 \\
\hline NGC5147 & -19.09 & SBd & 7.9 & 154.83 & 37.8 & 150.5 & 21.6 \\
\hline NGC5300 & -18.7 & $\mathrm{SABc}$ & 5.2 & 120.42 & 47.9 & 119.6 & 19.9 \\
\hline NGC5334 & -19.12 & $\mathrm{Sc}$ & 5.2 & 132.75 & 39.6 & 76.0 & 24.7 \\
\hline NGC5376 & -20.03 & $\mathrm{SABa}$ & 2.3 & 204.71 & 52.4 & 208.9 & 55.5 \\
\hline NGC5430 & -20.76 & $\mathrm{SBb}$ & 3.1 & 186.86 & 49.5 & 87.4 & 37.9 \\
\hline NGC5480 & -19.94 & $\mathrm{Sc}$ & 5.0 & 150.36 & 31.8 & 231.8 & 22.4 \\
\hline NGC5584 & -19.69 & $\mathrm{SABc}$ & 6.0 & 124.86 & 42.3 & 292.1 & 19.7 \\
\hline NGC5624 & -18.75 & Sbc & 3.8 & 66.52 & 48.7 & 71.8 & 35 \\
\hline NGC5660 & -20.66 & $\mathrm{SABc}$ & 5.2 & 138.52 & 18.2 & 129.6 & 37.2 \\
\hline NGC5667 & -19.93 & $\mathrm{SBc}$ & 6.0 & 100.4 & 58.0 & 100.1 & 34.8 \\
\hline NGC5668 & -20.01 & Scd & 6.9 & 72.52 & 31.8 & 134.0 & 26.9 \\
\hline NGC5693 & -19.08 & Scd & 6.9 & 44.83 & 18.2 & 139.6 & 40.1 \\
\hline NGC5713 & -21.16 & $\mathrm{SABb}$ & 4.0 & 107.91 & 29.5 & 81.6 & 18.3 \\
\hline NGC5768 & -19.43 & $\mathrm{Sc}$ & 5.3 & 123.62 & 27.1 & 337.4 & 33.1 \\
\hline
\end{tabular}

Table 1. Fundamental properties for the full sample. 


\begin{tabular}{llllllll} 
Galaxy & $M_{\mathbf{B}, \mathbf{a b s}}$ & $\mathbf{T y p e}$ & $\mathbf{t}$ & $v_{\mathbf{r o t}}[\mathrm{km} / \mathrm{s}]$ & $i\left[^{\circ}\right]$ & $\mathbf{P A}\left[^{\circ}\right]$ & $\mathbf{D}[\mathbf{M p c}]$ \\
\hline NGC5774 & -19.37 & SABc & 6,9 & 83.64 & 38.7 & 135.1 & 26.8 \\
NGC5806 & -19.92 & Sb & 3,2 & 190.93 & 57.3 & 104.7 & 25.2 \\
NGC5850 & -21.5 & Sb & 3,1 & 117.44 & 38.7 & 103.4 & 28.5 \\
NGC5937 & -21.17 & SABb & 3,2 & 180.29 & 55.9 & 70.9 & 46.6 \\
NGC6070 & -21.14 & Sc & 6,0 & 204.85 & 64.5 & 210.8 & 27.8 \\
NGC6155 & -20.02 & Sc & 5,2 & 109.64 & 43.9 & 133.3 & 41.7 \\
NGC7437 & -18.75 & SABc & 6,7 & 151.78 & 25.8 & 70.9 & 29.2 \\
NGC7606 & -21.2 & Sb & 3,0 & 445.74 & 67.7 & 125.7 & 31 \\
PGC006667 & -18.28 & Scd & 6,6 & 136.08 & 35.9 & 328.0 & 24.6 \\
UGC02081 & -18.39 & SABc & 5,8 & 95.56 & 54.5 & 108.8 & 42.5 \\
UGC04393 & -19.23 & Sbc & 3,5 & 62.21 & 56.6 & 29.0 & 15.2 \\
UGC06309 & -19.74 & SBc & 4,5 & 134.19 & 46.4 & 326.5 & 47 \\
UGC06518 & -19.06 & Sbc & 3,8 & 87.56 & 52.4 & 68.7 & 46.3 \\
UGC06903 & -18.35 & Sc & 5,9 & 163.69 & 28.4 & 131.9 & 30.5 \\
UGC07700 & -18.87 & Sd & 7,9 & 84.98 & 40.5 & 200.7 & 48.3 \\
UGC08041 & -18.48 & SBcd & 6,9 & 103.6 & 58.7 & 290.0 & 17.2 \\
UGC08084 & -18.84 & SBd & 8,0 & 88.77 & 34.9 & 210.5 & 41.1 \\
UGC08237 & -19.82 & SBb & 3,0 & & 38.7 & 138.5 & 47 \\
UGC08658 & -19.93 & Sc & 5,0 & 124.79 & 52.4 & 160.3 & 37.2 \\
UGC09741 & -18.84 & Sbc & 4,0 & & 27.1 & 102.3 & 42.9 \\
UGC09837 & -19.48 & SABc & 5,3 & 179.15 & 18.2 & 117.6 & 41.2 \\
UGC10721 & -19.72 & Sc & 5,8 & 143.07 & 47.9 & 159.8 & 45.8 \\
UGC12709 & -19.05 & SABm & 8,7 & 70.61 & 52.4 & 13.0 & 35.1 \\
\hline
\end{tabular}

Table 1. Fundamental properties for the full sample, continued.

rected images. Only in the case of some large galaxies, such as NGC 1042 and NGC 1068, did this approach fail and we were forced to remove these galaxies from our sample.

The mosaicking of images depends heavily on the correctness of the attached world coordinate system in each frame. The supplied coordinates were correct for all images, except for NGC 4210, where we found that stars were duplicated at multiple positions in the final mosaics. We corrected this using the solve-field tool from the astrometry.net project to verify and correct all headers automatically (Lang et al. 2010). This was done directly after downloading the raw SDSS images using mArchiveExec.

\subsection{Calibration}

Having created mosaics for the $g^{\prime}, r^{\prime}$ and $i^{\prime}$ bands, we need to calibrate them to $\mathrm{mag} / \operatorname{arcsec}^{2}$. Similar to Pohlen et al. (2004), we use the TsField table files associated with the original observation to get the photometric zero point $a a$, the extinction term $k k$ and the airmass coefficients. The surface brightness zero point is calculated as

$$
\begin{aligned}
\mu_{0}=\quad & -2.5 \times(0.4 \times[a a+k k \times \text { airmass }]) \\
& +2.5 \times \log _{10}\left(53.907456 \times 0.396^{2}\right),
\end{aligned}
$$

with an exposure time of $53.907456 \mathrm{~s}$ and an area per pixel of $0.396^{2} \operatorname{arcsec}^{2}$. The final surface brightness is then calculated as

$$
\mu=-2.5 \log _{10} \text { (counts) }+\mu_{0} .
$$

A series of reference stars was then selected in both the calibrated image and the mosaic. For both images, we measure the magnitudes of these stars. Using a linear fit to these magnitudes, the mosaic was then adjusted to match the calibration. On average around 15 stars were used, with a matching error below 0.05 magnitude.
As we noted before in Section 2, the PAS method has units of mag/arcsec rather than mag/ $\operatorname{arcsec}^{2}$, effectively making the value dependent on the projected size of the minor axis of the galaxy. We still follow the above calibration strategy for the PAS, but in all subsequent plots will add or subtract a linear constant term such that the least bright PAS profile (typically the $i^{\prime}$ ) starts at the same value as the brightest EP profile (typically the $g^{\prime}$ ). This is purely meant to guide the eye in direct comparisons between the various profiles and should not be seen as the true calibration.

\subsection{Centreing}

Michael Pohlen kindly provided us with the tables from Pohlen \& Trujillo (2006). We used the values therein to estimate the centre and position angle of the images, based on the 25th magnitude ellipse. The images were rotated to have their major axis aligned with the horizontal axis of the image. Overall this scheme worked well, and only in some cases did we have to tweak the position angle manually to better correspond to the image.

\subsection{Masking}

Foreground stars and background galaxies are a strong contaminant of the surface brightness profiles. Sextractor was used to create an initial set of masks, based on the $r^{\prime}$ band dataset. For masks outside the galaxy, set by the outer radius of the ellipse-fit profiles, we set the masked regions to zero. Doing that inside the galaxy would create holes in the profile, so a way to average over these parts was required. We therefore use IRAF package fixpix to interpolate the good parts of the image into the masked region. While far from perfect, this is the best solution for inner regions. If an object 


\begin{tabular}{|c|c|c|c|c|c|c|c|}
\hline \multirow[t]{2}{*}{ Galaxy } & \multicolumn{2}{|c|}{ Quality } & \multirow[t]{2}{*}{ Type } & \multicolumn{4}{|c|}{ Fit radii } \\
\hline & Image & Profile & & $r_{1}$ & $r_{2}$ & $r_{3}$ & $r_{4}$ \\
\hline IC1067 & G & M & I & 30 & 80 & & \\
\hline IC1125 & G & G & II & 30 & 40 & 45 & 60 \\
\hline IC1158 & G & G & II & 40 & 60 & 70 & 100 \\
\hline NGC0450 & $\mathrm{G}$ & $\mathrm{G}$ & II & 40 & 75 & 84 & 100 \\
\hline NGC0701 & G & $\mathrm{B}$ & I in ellipse, II in PAS & 20 & 60 & 60 & 80 \\
\hline NGC0853 & G & $\mathrm{B}$ & III in EP en Ell, I in PAS & 25 & 50 & 50 & 70 \\
\hline NGC0941 & $\mathrm{G}$ & $\mathrm{G}$ & II & 20 & 65 & 70 & 110 \\
\hline NGC1299 & G & M & II in PAS, III in EP and ellipse & 0 & 25 & 30 & 70 \\
\hline NGC2701 & $\mathrm{G}$ & G & II and III & 20 & 40 & 50 & 70 \\
\hline NGC2776 & G & $\mathrm{B}$ & II in PAS, III in EP and ellipse & 20 & 80 & 80 & 140 \\
\hline NGC2967 & G & $\mathrm{G}$ & III & 20 & 50 & 90 & 140 \\
\hline NGC3055 & $\mathrm{G}$ & G & II & 20 & 55 & 60 & 80 \\
\hline NGC3259 & G & $\mathrm{G}$ & III & 20 & 40 & 50 & 100 \\
\hline NGC3423 & G & G & II & 30 & 85 & 100 & 140 \\
\hline NGC3488 & $\mathrm{G}$ & M & II en 3 & 20 & 30 & 40 & 60 \\
\hline NGC3589 & G & G & II & 10 & 28 & 35 & 50 \\
\hline NGC3631 & G & M & II (EP fails due to variations) & 55 & 100 & 120 & 170 \\
\hline NGC3642 & G & M & III & 15 & 75 & 80 & 150 \\
\hline NGC3888 & $\mathrm{G}$ & M & II & 20 & 45 & 45 & 70 \\
\hline NGC3982 & G & G & III & 20 & 40 & 50 & 80 \\
\hline NGC4041 & $\mathrm{G}$ & $\mathrm{G}$ & III & 40 & 70 & 85 & 150 \\
\hline NGC4102 & G & $\mathrm{G}$ & II & 20 & 60 & 60 & 90 \\
\hline NGC4108 & G & B & II & 10 & 40 & 50 & 60 \\
\hline NGC4108B & $\mathrm{G}$ & $\mathrm{B}$ & I in ellipse, II in PAS & 0 & 40 & 40 & 55 \\
\hline NGC4273 & G & $\mathrm{B}$ & II & 40 & 60 & 80 & 120 \\
\hline NGC4545 & $\mathrm{G}$ & G & II & 20 & 60 & 60 & 70 \\
\hline NGC4653 & $\mathrm{G}$ & G & II & 20 & 80 & 105 & 125 \\
\hline NGC4668 & G & M & II en 3 & 20 & 33 & 40 & 50 \\
\hline NGC4904 & G & G & II & 15 & 35 & 50 & 80 \\
\hline NGC5147 & G & G & II & 7 & 35 & 40 & 70 \\
\hline NGC5300 & $\mathrm{G}$ & $\mathrm{G}$ & II & 13 & 75 & 100 & 140 \\
\hline NGC5334 & G & G & II & 40 & 80 & 100 & 140 \\
\hline NGC5376 & $\mathrm{G}$ & $\mathrm{G}$ & II & 10 & 30 & 40 & 70 \\
\hline NGC5430 & G & $\mathrm{G}$ & II & 30 & 48 & 60 & 90 \\
\hline NGC5480 & G & G & III & 20 & 40 & 80 & 120 \\
\hline NGC5624 & $\mathrm{G}$ & B & III in EP en Ell, I in PAS & 20 & 40 & 40 & 80 \\
\hline NGC5660 & $\mathrm{G}$ & M & II & 20 & 60 & 70 & 80 \\
\hline NGC5667 & $\mathrm{G}$ & $\mathrm{B}$ & II (EP fails due to variations) & 10 & 35 & 45 & 60 \\
\hline NGC5668 & $\mathrm{G}$ & $\mathrm{G}$ & II & 50 & 80 & 85 & 100 \\
\hline NGC5693 & G & $\mathrm{G}$ & II & 10 & 30 & 40 & 70 \\
\hline NGC5713 & G & $\mathrm{B}$ & I in ellipse, I in PAS & 50 & 85 & 100 & 130 \\
\hline NGC5774 & $\mathrm{G}$ & $\mathrm{G}$ & II & 20 & 80 & 85 & 130 \\
\hline NGC5806 & G & M & III & 50 & 100 & 150 & 200 \\
\hline NGC6155 & G & M & II & 0 & 30 & 40 & 60 \\
\hline NGC7437 & $\mathrm{G}$ & $\mathrm{G}$ & II & 20 & 40 & 50 & 80 \\
\hline PGC006667 & G & G & II & 30 & 70 & 80 & 110 \\
\hline UGC02081 & G & G & II & 0 & 55 & 60 & 90 \\
\hline UGC04393 & G & $\mathrm{B}$ & II & 40 & 60 & 60 & 70 \\
\hline UGC06309 & $\mathrm{G}$ & $\mathrm{B}$ & II & 20 & 40 & 40 & 60 \\
\hline UGC06518 & $\mathrm{G}$ & G & II & 10 & 25 & 25 & 38 \\
\hline UGC06903 & $\mathrm{G}$ & G & II & 20 & 50 & 60 & 95 \\
\hline UGC07700 & G & $\mathrm{G}$ & II & 20 & 39 & 49 & 80 \\
\hline UGC08084 & G & G & II & 11 & 40 & 45 & 60 \\
\hline UGC08658 & $\mathrm{G}$ & G & II & 20 & 49 & 55 & 90 \\
\hline UGC09741 & $\mathrm{G}$ & G & III & 10 & 20 & 25 & 40 \\
\hline UGC09837 & G & G & II & 20 & 45 & 53 & 67 \\
\hline UGC12709 & $\mathrm{G}$ & G & II & 31 & 75 & 75 & 100 \\
\hline
\end{tabular}

Table 2. Quality and fit radii for the approved image sample. Profile quality is split into types bad, moderate and good. Radii in arcsec. 


\begin{tabular}{|c|c|c|c|c|c|c|c|c|}
\hline \multirow[b]{2}{*}{ Galaxy } & \multicolumn{2}{|c|}{ Ellipse } & \multicolumn{6}{|c|}{ Equivalent Profiles } \\
\hline & $h_{0} \mathbf{r}$ & $h_{f} \mathbf{r}$ & $h_{0} \mathbf{r}$ & $h_{f} \mathbf{r}$ & $h_{0} \mathbf{g}$, & $h_{f} \mathbf{g}$, & $h_{0} \mathbf{i}$ & $h_{f} \mathbf{i}$ \\
\hline IC1125 & $2.64 \pm 0.05$ & $1.63 \pm 0.06$ & $2.48 \pm<0.01$ & $1.66 \pm<0.01$ & $2.72 \pm<0.01$ & $1.69 \pm<0.01$ & $2.38 \pm<0.01$ & $1.95 \pm<0.01$ \\
\hline IC1158 & $3.53 \pm 0.10$ & $1.49 \pm 0.03$ & $3.23 \pm<0.01$ & $1.51 \pm<0.01$ & $3.22 \pm<0.01$ & $1.66 \pm<0.01$ & $3.34 \pm<0.01$ & $1.69 \pm<0.01$ \\
\hline NGC0450 & $2.74 \pm 0.03$ & $1.32 \pm 0.07$ & $3.00 \pm<0.01$ & $1.35 \pm<0.01$ & $3.07 \pm<0.01$ & $1.35 \pm<0.01$ & $2.92 \pm<0.01$ & $1.42 \pm<0.01$ \\
\hline NGC0941 & $2.11 \pm 0.01$ & $1.48 \pm 0.04$ & $2.15 \pm<0.01$ & $1.71 \pm<0.01$ & $2.20 \pm<0.01$ & $1.71 \pm<0.01$ & $2.17 \pm<0.01$ & $1.95 \pm<0.01$ \\
\hline NGC2701 & $3.71 \pm 0.04$ & $1.20 \pm 0.01$ & $3.53 \pm<0.01$ & $1.22 \pm<0.01$ & $3.79 \pm<0.01$ & $1.20 \pm<0.01$ & $3.44 \pm<0.01$ & $1.40 \pm<0.01$ \\
\hline NGC2967 & $2.48 \pm 0.01$ & $5.50 \pm 0.13$ & $2.50 \pm<0.01$ & $5.56 \pm 0.01$ & $2.56 \pm<0.01$ & $5.77 \pm 0.02$ & $2.49 \pm<0.01$ & $5.95 \pm 0.01$ \\
\hline NGC3055 & $2.43 \pm 0.02$ & $1.30 \pm 0.02$ & $2.20 \pm<0.01$ & $1.18 \pm<0.01$ & $2.22 \pm<0.01$ & $1.17 \pm<0.01$ & $2.24 \pm<0.01$ & $1.22 \pm<0.01$ \\
\hline NGC3259 & $2.06 \pm 0.01$ & $4.83 \pm 0.09$ & $2.07 \pm<0.01$ & $4.81 \pm 0.01$ & $2.27 \pm<0.01$ & $4.86 \pm<0.01$ & $2.07 \pm<0.01$ & $5.10 \pm 0.01$ \\
\hline NGC3423 & $2.81 \pm 0.03$ & $0.95 \pm 0.01$ & $2.39 \pm<0.01$ & $1.01 \pm<0.01$ & $2.51 \pm<0.01$ & $1.02 \pm<0.01$ & $2.35 \pm<0.01$ & $1.04 \pm<0.01$ \\
\hline NGC3589 & $3.39 \pm 0.04$ & $1.59 \pm 0.02$ & $3.06 \pm<0.01$ & $1.49 \pm<0.01$ & $3.31 \pm<0.01$ & $1.39 \pm<0.01$ & $3.13 \pm<0.01$ & $1.64 \pm<0.01$ \\
\hline NGC3982 & $1.23 \pm 0.01$ & $1.90 \pm 0.04$ & $1.26 \pm<0.01$ & $1.94 \pm<0.01$ & $1.27 \pm<0.01$ & $2.08 \pm<0.01$ & $1.28 \pm<0.01$ & $1.90 \pm<0.01$ \\
\hline NGC4041 & $1.94 \pm 0.01$ & $3.22 \pm 0.05$ & $2.03 \pm<0.01$ & $3.93 \pm 0.01$ & $1.98 \pm<0.01$ & $4.05 \pm 0.01$ & $2.12 \pm<0.01$ & $4.49 \pm 0.01$ \\
\hline NGC4102 & $2.76 \pm 0.06$ & $1.19 \pm<0.01$ & $2.46 \pm<0.01$ & $1.14 \pm<0.01$ & $2.36 \pm<0.01$ & $1.15 \pm<0.01$ & $2.52 \pm<0.01$ & $1.16 \pm<0.01$ \\
\hline NGC4545 & $3.11 \pm 0.01$ & $2.21 \pm 0.10$ & $3.10 \pm<0.01$ & $2.16 \pm<0.01$ & $3.22 \pm<0.01$ & $2.00 \pm<0.01$ & $3.12 \pm<0.01$ & $2.25 \pm<0.01$ \\
\hline NGC4653 & $4.34 \pm 0.02$ & $2.74 \pm 0.14$ & $4.39 \pm<0.01$ & $3.30 \pm<0.01$ & $4.67 \pm<0.01$ & $3.10 \pm 0.01$ & $4.34 \pm<0.01$ & $3.31 \pm 0.01$ \\
\hline NGC4904 & $2.77 \pm 0.02$ & $1.26 \pm 0.01$ & $2.19 \pm<0.01$ & $1.20 \pm<0.01$ & $2.42 \pm<0.01$ & $1.15 \pm<0.01$ & $2.09 \pm<0.01$ & $1.25 \pm<0.01$ \\
\hline NGC5147 & $2.34 \pm 0.03$ & $1.22 \pm 0.01$ & $2.01 \pm<0.01$ & $1.22 \pm<0.01$ & $2.20 \pm<0.01$ & $1.18 \pm<0.01$ & $1.93 \pm<0.01$ & $1.25 \pm<0.01$ \\
\hline NGC5300 & $3.64 \pm 0.01$ & $1.68 \pm 0.02$ & $3.50 \pm<0.01$ & $1.87 \pm<0.01$ & $3.83 \pm<0.01$ & $1.82 \pm 0.01$ & $3.43 \pm<0.01$ & $1.94 \pm<0.01$ \\
\hline NGC5334 & $4.67 \pm 0.03$ & $2.21 \pm 0.11$ & $4.78 \pm<0.01$ & $2.34 \pm<0.01$ & $5.00 \pm<0.01$ & $2.40 \pm<0.01$ & $4.69 \pm<0.01$ & $2.57 \pm<0.01$ \\
\hline NGC5376 & $4.92 \pm 0.04$ & $3.05 \pm 0.01$ & $4.76 \pm<0.01$ & $3.11 \pm<0.01$ & $4.97 \pm 0.01$ & $3.09 \pm<0.01$ & $4.67 \pm<0.01$ & $3.22 \pm<0.01$ \\
\hline NGC5430 & $4.23 \pm 0.06$ & $2.14 \pm 0.04$ & $3.40 \pm<0.01$ & $2.31 \pm<0.01$ & $3.50 \pm<0.01$ & $2.37 \pm<0.01$ & $3.37 \pm<0.01$ & $2.43 \pm<0.01$ \\
\hline NGC5480 & $1.33 \pm 0.01$ & $2.91 \pm 0.14$ & $1.22 \pm<0.01$ & $1.91 \pm 0.02$ & $1.14 \pm<0.01$ & $1.83 \pm 0.02$ & $1.30 \pm<0.01$ & $1.65 \pm 0.03$ \\
\hline NGC5668 & $4.99 \pm 0.11$ & $3.20 \pm 0.02$ & $4.20 \pm<0.01$ & $3.35 \pm<0.01$ & $4.18 \pm<0.01$ & $3.09 \pm<0.01$ & $4.17 \pm<0.01$ & $3.71 \pm<0.01$ \\
\hline NGC5693 & $3.30 \pm 0.03$ & $1.78 \pm 0.05$ & $2.92 \pm<0.01$ & $2.02 \pm<0.01$ & $3.22 \pm<0.01$ & $1.89 \pm<0.01$ & $2.81 \pm<0.01$ & $2.10 \pm<0.01$ \\
\hline NGC5774 & $4.25 \pm 0.06$ & $3.08 \pm 0.05$ & $4.40 \pm<0.01$ & $3.18 \pm 0.01$ & $4.47 \pm 0.01$ & $3.01 \pm 0.01$ & $4.34 \pm<0.01$ & $3.39 \pm<0.01$ \\
\hline NGC7437 & $3.59 \pm 0.03$ & $2.18 \pm 0.02$ & $3.44 \pm<0.01$ & $2.25 \pm<0.01$ & $3.36 \pm<0.01$ & $2.15 \pm<0.01$ & $3.51 \pm<0.01$ & $2.36 \pm<0.01$ \\
\hline PGC006667 & $2.94 \pm 0.03$ & $1.48 \pm 0.07$ & $2.80 \pm<0.01$ & $1.75 \pm 0.01$ & $2.77 \pm<0.01$ & $1.40 \pm 0.01$ & $2.94 \pm<0.01$ & $2.04 \pm 0.01$ \\
\hline UGC02081 & $3.43 \pm 0.03$ & $1.73 \pm 0.15$ & $3.87 \pm<0.01$ & $2.92 \pm 0.01$ & $3.98 \pm<0.01$ & $2.91 \pm 0.02$ & $3.75 \pm<0.01$ & $3.06 \pm 0.02$ \\
\hline UGC06518 & $1.53 \pm 0.01$ & $1.36 \pm 0.04$ & $1.53 \pm<0.01$ & $1.38 \pm<0.01$ & $1.54 \pm<0.01$ & $1.31 \pm<0.01$ & $1.55 \pm<0.01$ & $1.45 \pm<0.01$ \\
\hline UGC06903 & $5.13 \pm 0.09$ & $1.50 \pm 0.04$ & $5.46 \pm<0.01$ & $1.80 \pm<0.01$ & $6.04 \pm<0.01$ & $1.68 \pm<0.01$ & $5.11 \pm<0.01$ & $1.97 \pm<0.01$ \\
\hline UGC07700 & $9.83 \pm 0.17$ & $2.18 \pm 0.12$ & $5.68 \pm<0.01$ & $2.92 \pm 0.01$ & $5.54 \pm<0.01$ & $2.74 \pm<0.01$ & $6.16 \pm<0.01$ & $3.43 \pm<0.01$ \\
\hline UGC08084 & $5.92 \pm 0.11$ & $1.63 \pm 0.09$ & $4.28 \pm<0.01$ & $2.09 \pm 0.01$ & $4.22 \pm<0.01$ & $1.98 \pm<0.01$ & $4.44 \pm 0.01$ & $2.40 \pm<0.01$ \\
\hline UGC08658 & $4.10 \pm 0.03$ & $2.92 \pm 0.03$ & $4.07 \pm<0.01$ & $3.17 \pm<0.01$ & $4.44 \pm<0.01$ & $3.24 \pm<0.01$ & $3.92 \pm<0.01$ & $3.35 \pm<0.01$ \\
\hline UGC09741 & $1.02 \pm 0.01$ & $2.55 \pm 0.03$ & $1.12 \pm<0.01$ & $2.48 \pm<0.01$ & $0.99 \pm<0.01$ & $2.55 \pm<0.01$ & $1.21 \pm<0.01$ & $2.48 \pm<0.01$ \\
\hline UGC09837 & $3.89 \pm 0.08$ & $1.39 \pm 0.05$ & $3.51 \pm<0.01$ & $1.67 \pm 0.01$ & $3.69 \pm<0.01$ & $1.52 \pm<0.01$ & $3.47 \pm<0.01$ & $1.99 \pm 0.01$ \\
\hline UGC12709 & $5.48 \pm 0.09$ & $2.15 \pm 0.09$ & $4.98 \pm 0.01$ & $2.61 \pm<0.01$ & $4.97 \pm 0.01$ & $2.16 \pm<0.01$ & $4.98 \pm<0.01$ & $2.88 \pm 0.01$ \\
\hline
\end{tabular}

Table 3. Derived scale lengths for the ellipse-fit and EP. Units in kpc. Errors are formal. The slopes of the PAS profiles are los-convlved and therefore not directly translatable into scale lengths, so we have omitted results from that method.

has not been fully masked, its unmasked pixels will contaminate the interpolation. An RGB (red-green-blue) image was therefore created from the three bands, and the quality of the masks was inspected. We tweak the mask by hand and recreate the RGB image. This process was repeated until we were satisfied with the result. In some cases, the contamination is too strong. We then resort to disabling those quadrants in the minor-axis integrated profiles. The Equivalent Profiles lack such a feature, and in some cases, they clearly suffer for it. For the worst cases, we therefore remove these galaxies from our sample.

As an alternative scheme for future work, it would also have been possible to replace the values of the masking with the expected values as measured through an initial ellipse fit. However, the advantage of using our fixpix solution is that we make use of the local structure of the galaxy, rather than introduce an idealized symmetric version of the galaxy.

\subsection{Background Subtraction}

Background subtraction is a famous problem in SDSS images, where due to the storage of numbers as integers one can only measure the background using very large samples (Pohlen \& Trujillo 2006). We perform a run of ELLIPSE on the data using the default parameters but with fixed inclination, centre and position angle. The background offset is taken as the mean value of all results between one and two times the $R_{\text {outer }}$. Here we use $R_{\text {outer }}$ to denote the outermost projected radius of our profile extraction region, which will cover a region well beyond the galaxy. The one-sigma background noise $\sigma$ is taken by measuring the standard deviation of all pixels in that same region. The uncertainty estimation is performed by using the PYTHON scipy.stats.bayes_mvs to perform a Bayesian fit of a normal distribution to the background. The uncertainty is based on the average confidence limit for the mean.

In the online Appendix A we present RGB images of the background, based on the three bands for selected galaxies. In regions of the image where an overlap occurs between two SDSS frames, there is a better signal to noise ratio due 
Radial Surface Photometry Profiles

\begin{tabular}{|c|c|c|c|c|c|c|c|c|c|c|c|c|c|c|}
\hline \multirow{3}{*}{$\frac{\text { Galaxy }}{\text { IC1125 }}$} & \multicolumn{3}{|c|}{ Ellipse } & \multicolumn{5}{|c|}{ EP } & \multicolumn{6}{|c|}{ PAS } \\
\hline & \multicolumn{2}{|l|}{$r_{f} \mathrm{r}^{\prime}$} & \multicolumn{2}{|l|}{$r_{f} \mathrm{r}^{\prime}$} & \multicolumn{2}{|c|}{$r_{f}$ g' } & \multicolumn{2}{|l|}{$r_{f} \mathrm{i}^{\prime}$} & \multicolumn{2}{|l|}{$r_{f} \mathrm{r}^{\prime}$} & \multicolumn{2}{|l|}{$r_{f}$ g' } & \multicolumn{2}{|l|}{$r_{f} \mathrm{i}^{\prime}$} \\
\hline & 6.978 & 0.166 & 6.910 & $<0.001$ & 6.656 & 0.003 & 6.147 & 0.003 & 7.050 & 0.066 & 7.095 & 0.027 & 7.484 & 0.067 \\
\hline IC1158 & 11.575 & 0.103 & 11.801 & 0.006 & 11.522 & 0.002 & 11.849 & 0.005 & 11.551 & 0.041 & 11.318 & 0.067 & 11.687 & 0.056 \\
\hline NGC0450 & 14.471 & 0.137 & 14.077 & 0.003 & 13.962 & 0.002 & 14.121 & 0.002 & 13.535 & 0.106 & 13.792 & 0.041 & 13.071 & 0.120 \\
\hline NGC0941 & 13.509 & 0.157 & 13.087 & 0.013 & 12.749 & 0.011 & 14.655 & 0.014 & 12.039 & 0.078 & 12.126 & 0.076 & 11.469 & 0.145 \\
\hline NGC2701 & 7.322 & 0.035 & 7.386 & 0.004 & 7.377 & 0.004 & 7.159 & 0.004 & 6.851 & 0.062 & 6.943 & 0.033 & 6.800 & 0.045 \\
\hline NGC2967 & 14.364 & 0.118 & 14.128 & 0.004 & 14.075 & 0.010 & 13.881 & 0.007 & 14.927 & 0.092 & 14.088 & 0.092 & 14.149 & 0.070 \\
\hline NGC3055 & 9.046 & 0.082 & 9.638 & $<0.001$ & 9.470 & 0.002 & 9.690 & 0.001 & 9.548 & 0.087 & 9.801 & 0.067 & 9.015 & 0.070 \\
\hline NGC3259 & 8.762 & 0.067 & 8.344 & 0.003 & 8.661 & 0.002 & 8.264 & 0.004 & 7.532 & 0.105 & 8.531 & 0.126 & 7.844 & 0.095 \\
\hline NGC3423 & 15.077 & 0.115 & 15.462 & 0.002 & 15.154 & 0.008 & 15.563 & 0.001 & 16.490 & 0.053 & 15.880 & 0.043 & 16.595 & 0.046 \\
\hline NGC3589 & 6.039 & 0.053 & 5.894 & 0.001 & 5.850 & 0.001 & 5.904 & $<0.001$ & 5.307 & 0.023 & 5.283 & 0.030 & 4.825 & 0.059 \\
\hline NGC3982 & 9.260 & 0.077 & 9.200 & 0.006 & 9.091 & 0.001 & 9.271 & 0.004 & 7.179 & 0.074 & 7.327 & 0.056 & 7.673 & 0.091 \\
\hline NGC4041 & 13.233 & 0.134 & 14.211 & 0.009 & 14.570 & 0.006 & 14.190 & 0.008 & 14.644 & 0.253 & 15.977 & 0.334 & 13.289 & 0.307 \\
\hline NGC4102 & 9.317 & 0.059 & 10.232 & $<0.001$ & 10.378 & 0.002 & 10.165 & $<0.001$ & 9.550 & 0.058 & 9.549 & 0.059 & 9.534 & 0.049 \\
\hline NGC4545 & 9.708 & 0.174 & 9.991 & 0.001 & 10.086 & 0.001 & 10.130 & 0.001 & 9.651 & 0.037 & 9.583 & 0.032 & 9.920 & 0.023 \\
\hline NGC4653 & 19.023 & 0.138 & 15.169 & 0.005 & 15.479 & 0.022 & 16.104 & 0.027 & 16.377 & 0.220 & 16.472 & 0.035 & 17.143 & 0.051 \\
\hline NGC4904 & 7.271 & 0.043 & 8.358 & 0.005 & 7.938 & 0.004 & 8.731 & 0.011 & 7.369 & 0.024 & 7.106 & 0.028 & 7.152 & 0.032 \\
\hline NGC5147 & 4.909 & 0.062 & 5.098 & 0.001 & 5.240 & $<0.001$ & 5.191 & 0.002 & 5.643 & 0.035 & 5.975 & 0.038 & 5.875 & 0.050 \\
\hline NGC5300 & 13.997 & 0.111 & 13.205 & 0.027 & 12.824 & 0.042 & 13.463 & 0.020 & 15.143 & 0.066 & 14.825 & 0.087 & 14.957 & 0.074 \\
\hline NGC5334 & 16.679 & 0.259 & 16.117 & 0.008 & 15.778 & 0.006 & 16.165 & 0.006 & 14.847 & 0.094 & 14.308 & 0.061 & 15.967 & 0.052 \\
\hline NGC5376 & 5.054 & 0.054 & 4.989 & 0.001 & 4.882 & 0.003 & 5.010 & 0.001 & 5.938 & 0.032 & 5.568 & 0.039 & 5.947 & 0.031 \\
\hline NGC5430 & 8.756 & 0.164 & 9.778 & 0.002 & 9.212 & 0.012 & 9.843 & 0.006 & 8.795 & 0.027 & 8.360 & 0.050 & 8.643 & 0.041 \\
\hline NGC5480 & 10.239 & 0.213 & 5.556 & 0.175 & 5.061 & 0.221 & -0.523 & 1.000 & 4.314 & 0.146 & 5.497 & 0.174 & 6.705 & 0.185 \\
\hline NGC5668 & 11.563 & 0.088 & 12.335 & 0.002 & 12.645 & 0.003 & 9.263 & 0.021 & 14.602 & 0.057 & 13.225 & 0.112 & 14.102 & 0.066 \\
\hline NGC5693 & 6.329 & 0.157 & 6.527 & 0.004 & 6.224 & 0.003 & 6.680 & 0.002 & 6.085 & 0.048 & 6.488 & 0.065 & 5.890 & 0.102 \\
\hline NGC5774 & 16.470 & 0.416 & 16.426 & 0.005 & 15.913 & 0.034 & 17.501 & 0.008 & 13.589 & 0.057 & 14.263 & 0.066 & 14.282 & 0.069 \\
\hline NGC7437 & 6.316 & 0.095 & 5.973 & 0.004 & 5.704 & 0.013 & 6.302 & 0.007 & 7.311 & 0.059 & 7.080 & 0.119 & 4.729 & 0.249 \\
\hline PGC006667 & 12.400 & 0.259 & 11.955 & 0.036 & 12.541 & 0.036 & 12.246 & 0.058 & 12.875 & 0.112 & 12.808 & 0.102 & 12.854 & 0.228 \\
\hline UGC02081 & 12.189 & 0.194 & 10.943 & 0.008 & 10.003 & 0.037 & 10.532 & 0.038 & 9.891 & 0.131 & 8.947 & 0.193 & 9.354 & 0.148 \\
\hline UGC06518 & 4.439 & 0.985 & 4.806 & 0.017 & 4.468 & 0.001 & 4.960 & 0.027 & 4.198 & 0.065 & 4.211 & 0.022 & 4.124 & 0.070 \\
\hline UGC06903 & 10.587 & 0.085 & 9.965 & 0.004 & 9.991 & 0.002 & 9.835 & 0.005 & 9.816 & 0.049 & 10.070 & 0.060 & 9.429 & 0.051 \\
\hline UGC07700 & 7.569 & 0.143 & 7.398 & 0.010 & 7.836 & 0.005 & 6.607 & 0.004 & 8.284 & 0.072 & 8.786 & 0.072 & 7.471 & 0.194 \\
\hline UGC08084 & 6.887 & 0.121 & 6.762 & 0.009 & 6.840 & 0.004 & 6.754 & 0.006 & 6.868 & 0.070 & 5.449 & 0.095 & 5.664 & 0.312 \\
\hline UGC08658 & 10.209 & 0.101 & 9.605 & 0.002 & 9.695 & 0.003 & 9.269 & 0.011 & 8.928 & 0.106 & 9.090 & 0.092 & 10.540 & 0.089 \\
\hline UGC09741 & 3.647 & 0.030 & 3.681 & 0.001 & 3.667 & 0.001 & 3.774 & 0.001 & 3.433 & 0.035 & 3.008 & 0.039 & 3.212 & 0.063 \\
\hline UGC09837 & 8.569 & 0.070 & 8.032 & 0.011 & 8.182 & 0.005 & 7.824 & 0.014 & 8.532 & 0.046 & 8.747 & 0.046 & 8.978 & 0.091 \\
\hline UGC12709 & 12.401 & 0.136 & 12.104 & 0.007 & 11.980 & 0.005 & 12.313 & 0.020 & 13.096 & 0.037 & 12.311 & 0.136 & 12.808 & 0.087 \\
\hline
\end{tabular}

Table 4. Derived break radii. Radii are given in arcsec.

to the double observing time spend at those positions. Since the position of the individual frames that make up an image are not identically placed, this leads to locally different colors in the RGB images. This is expected and we therefore do not worry about this. However, it does imply that the background does not have a constant noise level throughout the entire image. While this is not in itself a bad thing, it is worth keeping in mind when examining the profiles with regard to the uncertainty limit. The mosaicking also introduces (low) correlated noise by regridding (averaging) the pixels. After that, all images were inspected for the flatness of the background; we are left with a sample of 57 galaxies with a stable background.

\section{RESULTS}

\subsection{Classifications}

We classify the profiles by eye for each method. While there will always be some observer bias in the classification, we try to minimize this by only distinguishing into three main classes of profiles. We follow the basic classification scheme proposed by Erwin et al. (2008). The first class, Type I, is used to classify galaxies which exhibit a more-or-less constant exponential decay, as known from the work by Patterson (1940), de Vaucouleurs (1948) and Freeman (1970). The Type II profiles will denote all galaxies, which show a downward break/bend in its profile, similar to Freeman (1970). The Type III profiles refers to the so-called to the "anti-truncated" profiles, as first reported by Erwin et al. (2005). In these profiles, there is a steep decent of the light, followed by a less steep descent. Unlike Pohlen \& Trujillo (2006) and Erwin et al. (2008), we do not sub-classify these profiles further.

There is no fixed criteria to quantify at which point a profile shows so many features that it stops being a simple type I profile, and we tend to classify more galaxies as type II compared to Pohlen \& Trujillo (2006). Four galaxies are classified as type I, 40 galaxies are of type II and 16 are type III. Note that some galaxies are associated with multiple classifications simultaneously. Overall, the profile classification is the same, independent of the type of profile. Including the mixed types in each category, the ratios are $7 \%$ of the galaxies as type I, $70 \%$ as type II and/or $28 \%$ 


\begin{tabular}{|c|c|c|c|c|c|c|c|c|c|c|c|c|c|c|}
\hline \multirow[b]{2}{*}{ Galaxy } & \multicolumn{2}{|c|}{ Ellipse } & \multicolumn{6}{|c|}{ EP } & \multicolumn{6}{|c|}{ PAS } \\
\hline & $\mu_{f} \mathrm{r}^{\prime}$ & \pm & $\mu_{f} \mathrm{r}^{\prime}$ & \pm & $\mu_{f} \mathrm{~g}^{\prime}$ & \pm & $\mu_{f}$ i' & \pm & $\mu_{f} \mathrm{r}^{\prime}$ & \pm & $\mu_{f} \mathrm{~g}^{\prime}$ & \pm & $\mu_{f}$ i' & \pm \\
\hline IC1125 & 23.613 & 0.075 & 23.630 & $<0.001$ & 23.971 & 0.001 & 23.025 & 0.001 & 18.822 & 0.077 & 19.301 & 0.033 & 18.978 & 0.034 \\
\hline IC1158 & .980 & 0.042 & 24.0 & 0.002 & 24.541 & 0.001 & & & 77 & & 19.261 & & & 039 \\
\hline NGC0450 & 1.061 & 0.029 & 23.899 & 0.001 & 24.169 & 0.001 & 23.766 & $<0.001$ & & & 18.304 & & 17.870 & .060 \\
\hline NGC0941 & 24.798 & 0.074 & 24.684 & 0.006 & 24.911 & 0.005 & 24.959 & 0.004 & 18.834 & 0.072 & 19.440 & 0.040 & 18.306 & .046 \\
\hline NGC2701 & 2.246 & 0.020 & 22.265 & 0.002 & 22.604 & 0.002 & 21.956 & 0.002 & 16.656 & 0.016 & 16.979 & 0.016 & 16.482 & .012 \\
\hline NGC2967 & 24.819 & 0.036 & 24.663 & 0.001 & 25.084 & 0.003 & 24.275 & 0.003 & 19.341 & 0.061 & 19.209 & 0.045 & 18.656 & .058 \\
\hline NGC3055 & 22.907 & 0.044 & 23.265 & $<0.001$ & 23.613 & 0.001 & 23.035 & 0.001 & 18.369 & 0.042 & 19.047 & 0.057 & 17.744 & .058 \\
\hline NGC3259 & 24.295 & 0.029 & 23.908 & 0.001 & 24.369 & 0.001 & 23.608 & 0.002 & 18.282 & 0.022 & 19.025 & 0.030 & 18.068 & 0.034 \\
\hline NGC3423 & 2.941 & 0.031 & 23.066 & 0.001 & 23.463 & 0.002 & 22.913 & $<0.001$ & 17.314 & 0.015 & 17.541 & 0.021 & 17.166 & 0.012 \\
\hline NGC3589 & 3.177 & 0.029 & 23.096 & $<0.001$ & 23.357 & $<0.001$ & 22.906 & $<0.001$ & 18.158 & 0.017 & 18.429 & 0.034 & 17.712 & 0.029 \\
\hline NGC3982 & 24.042 & 0.035 & 23.944 & 0.003 & 24.306 & 0.001 & 23.683 & 0.002 & 17.421 & 0.026 & 17.899 & 0.022 & 17.269 & 0.050 \\
\hline NGC4041 & 24.257 & 0.024 & 24.437 & 0.002 & 25.051 & 0.001 & 24.064 & 0.002 & 18.151 & 0.034 & 18.993 & 0.057 & 33 & 0.097 \\
\hline NGC4102 & 21.618 & 0.015 & 21.853 & $<0.001$ & 22.592 & 0.001 & 21.436 & $<0.001$ & 16.472 & & 17.140 & & & 0.018 \\
\hline NGC4545 & 23.526 & 0.0 & 23.6 & 0.001 & 24.094 & $<0.00$ & 23.4 & $<0.001$ & & & 18.652 & & & 0.012 \\
\hline NGC4653 & 26.072 & 0.067 & 24.659 & 0.002 & 25.234 & 0.009 & 24.756 & 0.011 & 18.999 & & 19.307 & 0.012 & 29 & .022 \\
\hline NGC4904 & .069 & 0.022 & 22.509 & 0.002 & 22.824 & 0.002 & 22.391 & 0.005 & 16.875 & 0. & 17.288 & 0.013 & 16.524 & 0.010 \\
\hline NGC5147 & .211 & 0.007 & 21.410 & $<0.001$ & 21.810 & $<0.001$ & 21.259 & 0.001 & 16.450 & 0.019 & 16.936 & 0.020 & 16.369 & 0.032 \\
\hline NGC5300 & 23.249 & 0.028 & 23.044 & 0.006 & 23.405 & 0.008 & 22.826 & 0.005 & 17.945 & 0.025 & 18.299 & 0.006 & 17.537 & 0.015 \\
\hline NGC5334 & 23.976 & 0.051 & 23.735 & 0.002 & 24.139 & 0.001 & 23.448 & 0.001 & 17.308 & 0.042 & 17.501 & 0.048 & 17.378 & 0.011 \\
\hline NGC5376 & 21.286 & 0.022 & 21.248 & $<0.001$ & 21.838 & 0.001 & 20.895 & $<0.001$ & 16.789 & 0.009 & 17.287 & 0.017 & 16.388 & 0.014 \\
\hline NGC5430 & 22.893 & 0.079 & 23.359 & 0.001 & 23.656 & 0.005 & 23.007 & 0.002 & 17.348 & 0.013 & 17.610 & 0.016 & 16.948 & 0.016 \\
\hline NGC5480 & 24.281 & 0.120 & 22.080 & 0.117 & 22.308 & 0.159 & & & 16.226 & 0.100 & 17.649 & 0.119 & 17.268 & 0.080 \\
\hline NGC5668 & 23.244 & 0.016 & 23.372 & 0.000 & 23.771 & 0.001 & 22.580 & 0.004 & 17.790 & 0.027 & 17.933 & 0.010 & 17.447 & 0.012 \\
\hline NGC5693 & 23.676 & 0.111 & 23.634 & 0.002 & 23.942 & 0.001 & 23.495 & 0.001 & 18.168 & 0.029 & 18.877 & 0.043 & 17.717 & 0.048 \\
\hline NGC5774 & 24.649 & 0.118 & 24.527 & 0.001 & 24.812 & & & & & & 18.524 & & 17.832 & 0.020 \\
\hline NGC7437 & .899 & 0.0 & & & & & & & & & 18.280 & & & 0.063 \\
\hline PGC006667 & 24.563 & 0.111 & 24.418 & 0.013 & 25.049 & 0.016 & 24.195 & 0.020 & 19.128 & 0.048 & 19.369 & 0.065 & 18.765 & 0.062 \\
\hline UGC02081 & 25.691 & 0.113 & 25.127 & 0.003 & 25.192 & 0.017 & 24.760 & 0.018 & 19.652 & 0.113 & 19.687 & 0.116 & 19.201 & 0.061 \\
\hline UGC06518 & 23.739 & 0.229 & 23.969 & 0.016 & 24.098 & 0.001 & 23.833 & 0.030 & 19.063 & 0.067 & 19.391 & 0.020 & 18.626 & 0.088 \\
\hline UGC06903 & 23.853 & 0.038 & 23.763 & 0.001 & 24.153 & 0.001 & 23.503 & 0.002 & 18.093 & 0.003 & 18.578 & 0.017 & 17.708 & 0.019 \\
\hline UGC07700 & 24.121 & 0.071 & 24.053 & 0.004 & 24.515 & 0.002 & 23.615 & 0.002 & 19.245 & 0.021 & 19.729 & 0.030 & 18.862 & 0.107 \\
\hline UGC08084 & 24.052 & 0.052 & 24.012 & 0.005 & 24.400 & 0.002 & 23.737 & 0.003 & 18.974 & 0.031 & 18.650 & 0.014 & 18.213 & 0.165 \\
\hline UGC08658 & 23.930 & 0.032 & 23.698 & 0.000 & 24.086 & 0.001 & 23.388 & 0.004 & 18.187 & 0.043 & 18.561 & 0.006 & 18.408 & 0.032 \\
\hline UGC09741 & 23.260 & 0.026 & 23.250 & 0.000 & 23.839 & 0.000 & 22.982 & 0.000 & 18.265 & 0.026 & 18.631 & 0.025 & 17.850 & 0.036 \\
\hline UGC09837 & 24.615 & 0.029 & 24.403 & 0.005 & 24.716 & 0.003 & 24.135 & & 19.087 & 0.020 & 19.612 & 0.023 & 19.308 & 0.085 \\
\hline UGC12709 & 25.177 & 0.054 & 25.140 & 0.002 & 25.464 & 0.002 & 24.967 & 0.006 & 20.017 & 0.034 & 20.339 & 0.127 & 19.809 & 0.046 \\
\hline
\end{tabular}

Table 5. Derived break colors. Break colors are given in ABmag/arcsec ${ }^{2}$.

as type III. Comparing this with the original classifications by Pohlen \& Trujillo (2006), their classifications would have been $12 \%$ in type I, $65 \%$ in type II and $35 \%$ in type III. 29 galaxies in this sample are classified by us as purely type II galaxies, of which four have been classified by Pohlen \& Trujillo (2006) as type I and five have been classified by them as both types II and III. In total, we match the complete classification by Pohlen \& Trujillo (2006) for 40 out of 57 galaxies $(70 \%)$.

The type III galaxies are an interesting set. Most of these galaxies show clear signs of interaction, be it an asymmetric disc (e.g. NGC853, Figure A6) or tidal tails (e.g. NGC3631, Figure A22 NGC3642, Figure A23. This has already been reported by van der Kruit \& Freeman (2011), who noted that many of the type III galaxies are mergers. Table 2 in the online Appendix shows all classifications.

\subsection{Measurement of Scale Lengths and Feature Radii}

Measurement of the scale lengths was done in the following way. For each galaxy we have seven profiles ( $r^{\prime}$ ellipse, and $g^{\prime}, r^{\prime}$ and $i^{\prime}$ for both EP and PAS). We select the most prominent feature in the profile by eye and try to define a fit-region on either side of the feature, where the profiles are showing more-or-less linear behavior. For the region before the feature, its outer radii are denoted by $R_{1}$ and $R_{2}$, while after the feature the radii are $R_{3}$ and $R_{4}$. Table $2 \mathrm{n}$ the online Appendix shows all these radii.

Knapen \& van der Kruit (1991) showed that the choice of radii to which a profile is fitted, has a strong effect on the derived scale lengths. To avoid the introduction of a bias, we use the same region for all of our profiles. This can result in a different position for the feature per technique, and in some cases we find that the fitting favors different features entirely. We therefore classify the overall goodness-of-fit for the entire fitting result by eye. We only focus our statistical analysis on the galaxies having goodness-of-fit quality flag 'G' and a purely type II profile. This limits our statistics sample to 29 galaxies. The scale lengths are listed in Table 3

We denote the scale lengths measured before the feature as the inner scale length $h_{0}$ and after the feature as outer scale length $h_{f}$. The feature radius is denoted by $R_{f}$, where any additional subscript will be used to refer to the specific method used. The feature surface brightness $\mu$ is measured 
as the surface brightness of the profile at the feature radius $R_{f}$. The radii and surface brightness are listed per galaxy in Table 4. The colours are listed in table 5

The following method is used to estimate the errors. From each fit region, we randomly drop $10 \%$ of the points. A linear fit is performed to the remaining $90 \%$. In cases where multiple fit regions are defined, we perform this simultaneous in every region. The resulting fits will be slightly different compared to the original fits. By proxy, the resulting feature radii and feature surface brightness will also be different. By repeating this process 100 times, a scatter distribution builds up for each variable. We measure the standard deviation from each distribution, and define the error as half-width half-maximum by multiplying it by $\sqrt{2 \ln 2}$. In some cases, we find that not all fits return realistic result. This occurs in particular in the more noisy regions. When this occurs, the error-bars are denoted using arrows in all subsequent graphics.

\subsection{Sub-classification into Breaks and Truncations}

After studying a sample of edge-on galaxies, Martín-Navarro et al. (2012) proposed to distinguish between breaks and truncations based on the criteria $R_{f} / h_{f}=5$. All values above five were considered truncations, while all values lower were considered breaks. We show the histogram of $R_{f} / h_{f}$ for our sample in Figure 4 Twelve truncations are found in the PAS sample. Seven galaxies in the EP profiles can be considered truncations, out of which six overlap with the PAS sample. We list the truncations sample in Table 6 along with pointers to the figures of the individual galaxies in the online Appendix. For clarity we repeat that we only include here the galaxies having goodness-of-fit quality flag ' $G$ ' and a purely type II profile.

Overall, we see that most of the $\mathrm{EP} R_{f} / h_{f}$ values lie in a compact range from 1.5 onto 6 . The PAS values span a wider range, between 1.0 and 10, and are more evenly spread. In the edge-on sample from Martín - Navarro et al. (2012) the $R_{f} / h_{f}$ ranges from 1 onto 20 . The mean values of $R_{f} / h_{f}$ for the ellipse-fit profiles is $4.46 \pm 2.86$, for the EP $4.02 \pm 1.67$ and for the PAS $4.71 \pm 5.14$. The wider spread of the PAS appears to represent edge-on profiles better than the EP profiles. It is interesting to note that the EP profiles have a lower average value and scatter than the ellipse-fit profiles. This is due to the redistribution of light that occurs as part of the EP method, which has the effect of smoothing out the profile a bit. In Figure 5 we show boxplots for all three distributions of $R_{f} / h_{f}$. As can be seen more clearly from this figure, the median values of the $R_{f} / h_{f}$ distributions are similar between the three distributions. We perform an ANOVA test on the three distributions of $R_{f} / h_{f}$, using $\mathrm{R}$, to test if the difference in variance is statistically significant. We find an F-value of 1.356 , which is not statistically significant.

\subsection{Correlation Tests}

Following Pohlen \& Trujillo (2006) we perform a range of correlation tests on all our parameters. We use the Spear-

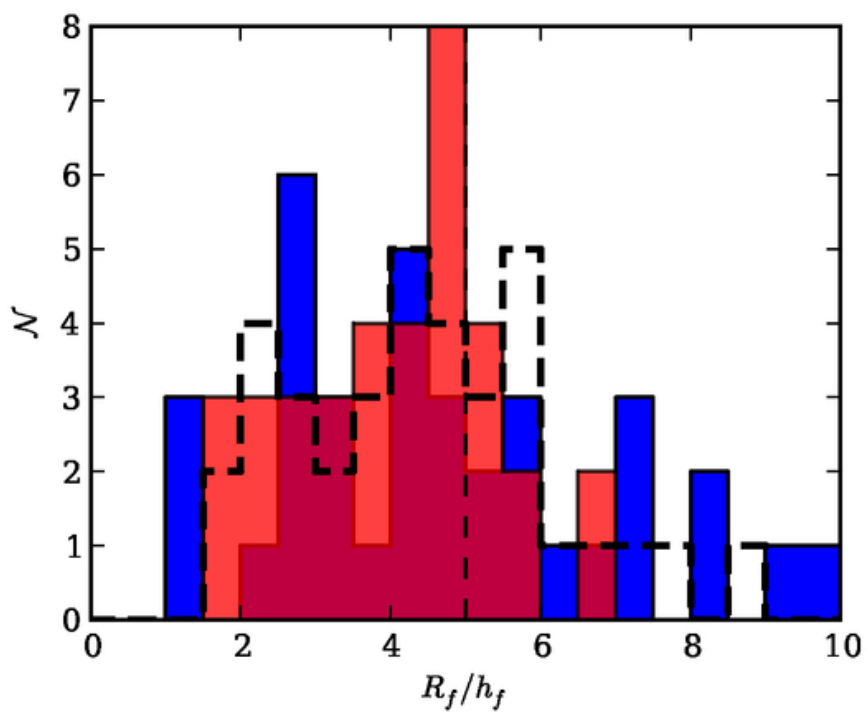

Figure 4. Histogram of $R_{f} / h_{f}$ for the final sample. The dashed vertical line represents the $R_{f} / h_{f}=5$ threshold proposed by Martín-Navarro et al. (2012), with all points to the left considered breaks and all points to the right considered truncations. In light red denotes the EP profiles, while the darker blue denote the PAS profiles. The thick dashed profile shows the distribution of the ellipse-fit profiles.

\begin{tabular}{lccr} 
Galaxy & In EP? & In PAS? & Figure \\
\hline IC1125 & $\times$ & $\times$ & A2 \\
IC1158 & & $\times$ & A3 \\
NGC0450 & $\times$ & $\times$ & A4 \\
NGC0941 & & $\times$ & \\
NGC2701 & $\times$ & & \\
NGC3055 & $\times$ & $\times$ & \\
NGC3423 & $\times$ & $\times$ & \\
NGC4545 & $\times$ & $\times$ & \\
NGC4653 & $\times$ & $\times$ \\
NGC5300 & & $\times$ \\
NGC5430 & & $\times$ \\
PGC006667 & & $\times$ \\
UGC08084 & & $\times$ \\
UGC09837 & $\times$ & $\times$ \\
UGC12709 & & \\
\hline
\end{tabular}

Table 6. List of truncations based on the Martín- Navarro et al. (2012) criterion

man rank correlation coefficient ${ }^{3} \rho$ to estimate if there is any monotone correlation between two parameters. We also calculate the corresponding significance $p$ of that $\rho$, using as a null-hypothesis the absence of correlation. We reject the null-hypothesis when $p<0.05$. The significance test $p$ only describes the chance of finding a particular value of $\rho$ less than or equal to the observed value purely by chance. Because of small number statistics, very strong correlations need few samples to become statistically significant. Fainter, but potentially real, correlations require far more samples to
3 We calculate the Spearman rank correlation coefficient $\rho$ and significance $p$ using the PYTHON package scipy.stats.spearmanr. 


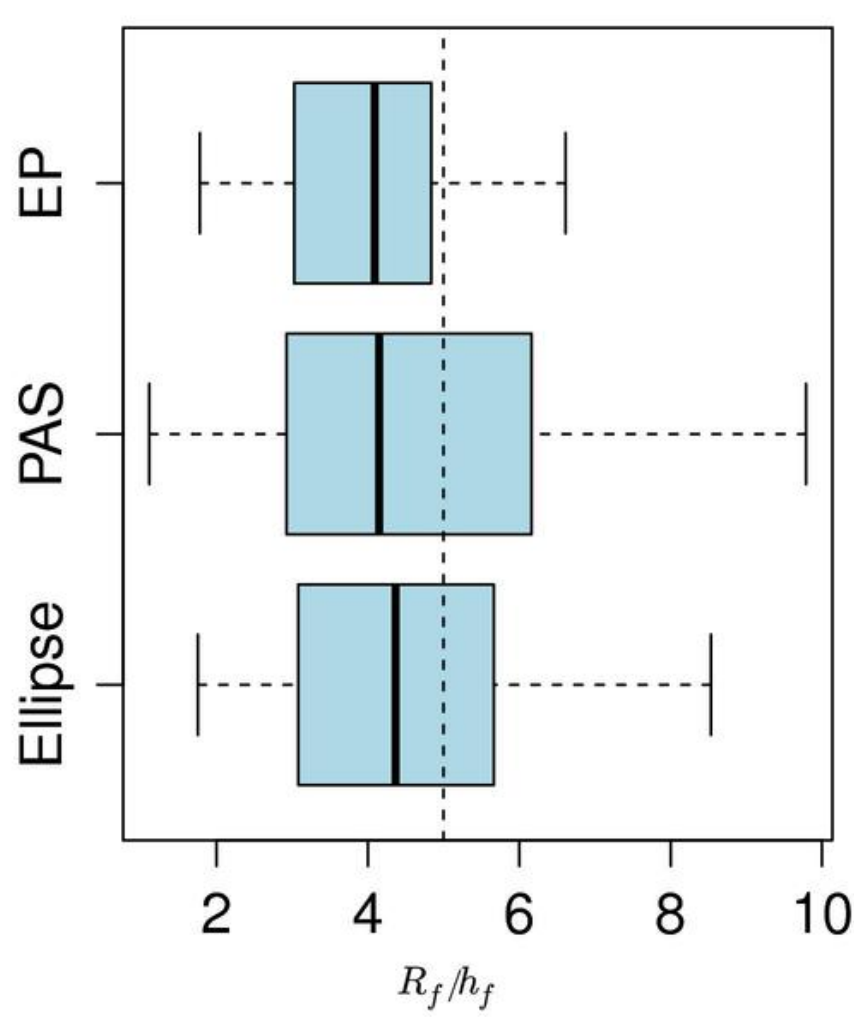

Figure 5. Boxplot of $R_{f} / h_{f}$ for the final sample. Thick vertical stripes denote the median values, the boxes denote the outlines of the $25 \%$ and $75 \%$, and the whiskers the minimum and maximum. The dashed vertical line represents the $R_{f} / h_{f}=5$ threshold proposed by Martín-Navarro et al. (2012), with all points to the left considered breaks and all points to the right considered truncations.

distinguish from random noise. As our sample is small, we can only report on relatively strong correlations.

In all subsequent figures, we print the correlation of the combined feature set. The symbols distinguish between breaks and truncations using filled and open markers. The full subset correlation tests can be found in Table 7 in the online Appendix. We will discuss the various correlations in the following subsections. Only the most prominent correlations are shown in figures.

\subsubsection{Scale lengths and radii}

The $r^{\prime}$-band inner and outer scale lengths, are shown in Figures 6 and 7 From Figure 6 we find that inner scale lengths, as measured in both the EP and PAS profiles compared to the ellipse-fit, follow very tight positive correlations. This holds for both the full sample and the sub-samples of truncations and breaks. The PAS profile have slightly more scatter, and thus the correlations are weaker than those of the EP with the ellipse-fit method. A line is fit through the data, using absolute differences as the cost function, and forcing the line through $(0,0)$. We find that $h_{0, \mathrm{EP}} \sim 0.95 h_{0, \mathrm{ell}}$ and $h_{0, \mathrm{PAS}} \sim 1.11 h_{0, \mathrm{ell}}$. The first relation is likely a result of the inability of the EP method to deal with bumps that the ellipse-fit can show. If a bump is present in the ellipse-fit profile, this can make the scale length in the ellipse-fit pro-

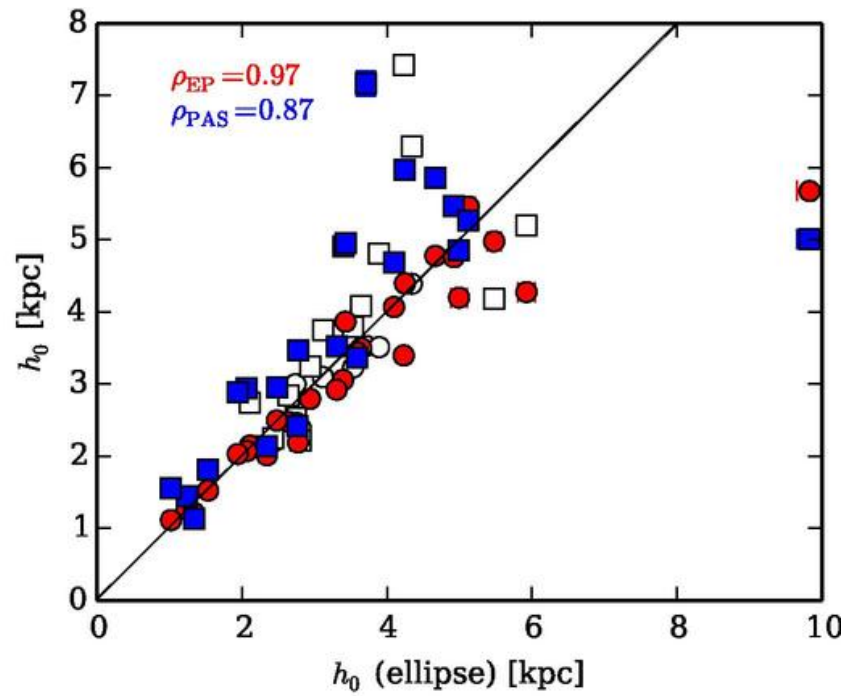

Figure 6. The inner scale length of the linear fits to the $r^{\prime}$ band profile, $h_{0}$, compared to the scale lengths as derived from the ellipse-fit profiles. The circles denote the EP profiles and the squares denote the PAS profiles. Filled markers are breaks, while open markers are likely truncations. The correlation significance $p$ are also shown.

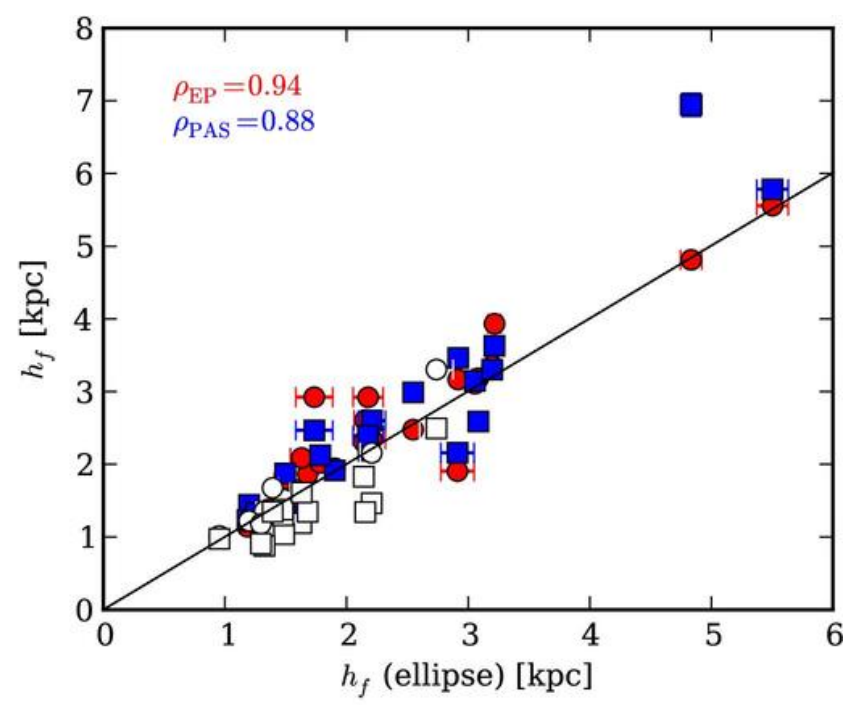

Figure 7. The outer scale length of the linear fits to the $r^{\prime}$ band profile, $h_{f}$, compared to the scale lengths as derived from the ellipse-fit profiles. The circles denote the EP profiles and the squares denote the PAS profiles. Filled markers are breaks, while open markers are likely truncations. The correlation significance $p$ are also shown.

file slightly steeper than that of the EP profiles, at least at larger radii than the bump.

Another point to notex is more profound: inner scale length of the PAS are longer than the profiles in ellipse-fit profiles. Likely this result is due to the geometry of the galaxies. The consequence is that in an edge-on galaxy without dust, the inner scale lengths will be longer. The mean inner scale length $h_{0}$ for the EP was $3.16 \pm 1.18 \mathrm{kpc}$ and very similar $3.16 \pm 1.30 \mathrm{kpc}$ and $3.17 \pm 0.64 \mathrm{kpc}$ for the breaks 
and truncations sub-samples. The mean inner scale length measured with the PAS is higher and has more scatter at $3.87 \pm 1.59 \mathrm{kpc}$, with again rather similar sub-samples at $3.81 \pm 1.65 \mathrm{kpc}$ and $3.95 \pm 1.49 \mathrm{kpc}$. The mean inner scale length of the ellipse-fit profiles was $3.46 \pm 1.64 \mathrm{kpc}$. This is very similar to Pohlen \& Trujillo (2006), who found a mean scale length $h_{0}$ of $3.8 \pm 1.2 \mathrm{kpc}$.

The same tight correlations with the ellipse-fit profiles remain for the scale lengths after the feature for both profiles. The biggest scatter increase occurs in the truncations sample of the PAS profiles, where $\rho_{\mathrm{PAS} \text {,truncations }}=$ 0.732 . This result however remains comfortably significant at $p<0.005$. The mean outer scale length $h_{f}$ as measured through the EP profiles was $2.26 \pm 1.03 \mathrm{kpc}$ for the EP methods. The breaks have $2.43 \pm 1.04 \mathrm{kpc}$ and the truncations $1.68 \pm 0.70 \mathrm{kpc}$. For the PAS we have found $h_{f}=2.13 \pm 1.28 \mathrm{kpc}$, with subdivisions into $2.61 \pm 1.41 \mathrm{kpc}$ and $1.36 \pm 0.41$. The average ellipse outer scale length $h_{f}$ is $2.12 \pm 0.98 \mathrm{kpc}$, with the breaks at $2.40 \pm 1.08 \mathrm{kpc}$ and the truncations at $1.61 \pm 0.47$. The outer truncation scale lengths of the EP and ellipse-fit profiles thus tend to be longer than their PAS counterparts. In edge-on galaxies, the scale length for a truncation is even shorter, at only $1.5 \pm 0.1 \mathrm{kpc}$. The break scale lengths is similar at $2.7 \pm 0.3 \mathrm{kpc}$ (MartínNavarro et al. 2012).

As we noted before, the PAS surface brightness levels are in magnitudes / arcsec rather than magnitudes / $\operatorname{arcsec}^{2}$, making it distance dependent. As a quick test to see if the distance could be a complicating fact in the increased scatter of the PAS, we model a very simple galaxy consisting of no more than a face-on, truncated exponential disk. We have placed at various distances, thus changing the number of points available in the minor axis summation. In this test, we found that the ratio between the ellipse derived scale lengths versus the PAS derived scale lengths did change with distance, but the scatter was below $1 \%$ of the derived scale lengths. Thus, distance does not affect the derived scale lengths significantly.

The feature radii from the ellipse-fit profiles to the feature radii measured in the other profiles for the r' band images, are also tightly correlated between the various methods. This is expected, as any other result would have caused the profile to be flagged. Again fitting this relation with a linear approximation we find that the both the EP and PAS profiles follow $h_{1, \mathrm{PAS} \& \mathrm{EP}}=1.03 h_{f \text {,ell }}$. The mean radius $R_{f}$ for the EP is $8.38 \pm 2.96 \mathrm{kpc}$ in the whole sample. The radii $R_{f}$ for the breaks and truncations sub samples are $8.13 \pm 2.77$ and $9.29 \pm 3.39 \mathrm{kpc}$. In comparison, the PAS profile radii $r_{f}$ have a mean position of $8.32 \pm 3.15 \mathrm{kpc}$, with the break and truncation sub samples at $7.61 \pm 3.02 \mathrm{kpc}$ and $9.44 \pm 3.03$ kpc. The EP results are similar to Pohlen \& Trujillo (2006), who report a typical radius of $R_{f}=9.2 \pm 2.4 \mathrm{kpc}$ for their type II-CT sample and $R_{f}=9.5 \pm 6.5$ for their OLR sample. An average radius of $7.9 \pm 0.9 \mathrm{kpc}$ was reported for the inner breaks in the edge-on sample of Martín-Navarro et al. (2012), while the average truncation was found at $14 \pm 2$ kpc.

Looking at the radius of the feature in terms of the number of (inner) scale lengths $R_{f} / h_{0}$, we have a mean of $2.84 \pm 0.98$ for the EP and $2.32 \pm 0.75$ for the PAS. This difference is a reflection of the result previously reported that PAS profiles tend to have longer inner scale lengths compared to the ellipse-fit and EP. The breaks and truncations sub samples for the breaks show similar behavior. For the EP we find $2.83 \pm 1.06$ for the breaks and $2.90 \pm 0.67$ for the truncations. For the PAS we find $2.20 \pm 0.83$ and $2.52 \pm 0.57$. Overall, we see that the truncations suffer from less scatter than the breaks. For truncations in edge-on galaxies, $r_{f} / h_{0}$ is expected to lie around $4.2 \pm 0.6$ (van der Kruit \& Searle 1982a) or $2.9 \pm 0.7$ (Pohlen et al. 2000). Sixteen face-on galaxies from the sample of Wevers (1984); Wevers et al. (1984) were analyzed by van der Kruit (1988) for the presence of truncations, who found $r_{f} / h_{0}=4.5 \pm 1.0$. Bosma \& Freeman (1993) argued that a large range of radii could be found, as seven galaxies in the Wevers et al. (1984) sample, have a relatively bright 'edge' at $r_{f} / h_{0}=2.8 \pm 0.4$, while his other did not show this and would thus have $R_{f} / h_{0}>4$. Pohlen et al. (2002) find for three galaxies a result of $3.9 \pm 0.7$. Pohlen \& Trujillo (2006) find a far lower $R_{f} / h_{0} \sim 2.5 \pm 0.6$ for their type II-CT sample and 1.7 for their breaks sample, which we argue in more detail in Peters et al. (2015) is due to different definitions of what is a break and truncation plus the way to mark this. There also remains the question of the consistency in measuring scale lengths in face-on galaxies and recovering these from projected data of edge-ons. In the literature there are thus studies indicating average values around 4 and less than 3 , and we see for the moment no consensus appearing. The indicative value of $3.5-4$ from fig.'s 1 and 2 in Kregel \& van der Kruit (2004) would seem in the light of this discussion not to do justice to the small values found and we revise our indicative value to $3-4$.

\subsubsection{Sharpness of the breaks}

In Figure 8, the 'sharpness' of the break is shown as measured through the ratio of inner over outer scale lengths $h_{0} / h_{b}$. Breaks from the EP range in strength from just above 0 until 3.5, while the PAS range up until 5. The average EP break sharpness is $h_{0} / h_{b}=1.57 \pm 0.64$, while the PAS is at $h_{0} / h_{b}=2.18 \pm 0.84$. This reflects the difference between face-on and edge-on profiles, where edge-on profiles typically have sharper features, going up to 12 (Martín-Navarro et al. 2012). Similar to our EP results, Pohlen \& Trujillo (2006) found $h_{0} / h_{b}=2.1 \pm 0.5$, ranging from 1.3 to 3.6 , in the type II-CT sample. Our more extensive classification into type II galaxies creates the difference at the lower end of this range. Truncations and breaks overlap in both profile types, although the truncations tend to lie only on higher values. Compared to the EP, the PAS has a wider and more spread, and can thus reach a wider range of sharpness levels.

Figure 8 offers an interesting alternative to the MartínNavarro et al. (2012) classification scheme of $R_{f} / h_{f}=5$. As can be seen from the figure, the truncations -as classified using the $R_{f} / h_{f}=5$ in the PAS- are all at high values of $h_{0} / h_{f}$. There are however also galaxies that did not meet the $R_{f} / h_{f} \geqslant 5$ criterion, but which still have high values of $h_{0} / h_{f}$. An alternative scheme could therefore be to classify all galaxies as having a truncation when the sharpness of a break is above some threshold. Observing the figure, a value of $h_{0} / h_{b}=2$ would seem appropriate, although we admit thsi has a certain level of arbitrariness.

In Figure 9, we plot the relation of $h_{f}$ with $R_{f} / h_{f}$. There is a very clear anti-correlation between the two. Schaye (2004) predicted the presence of an anti-correlation 


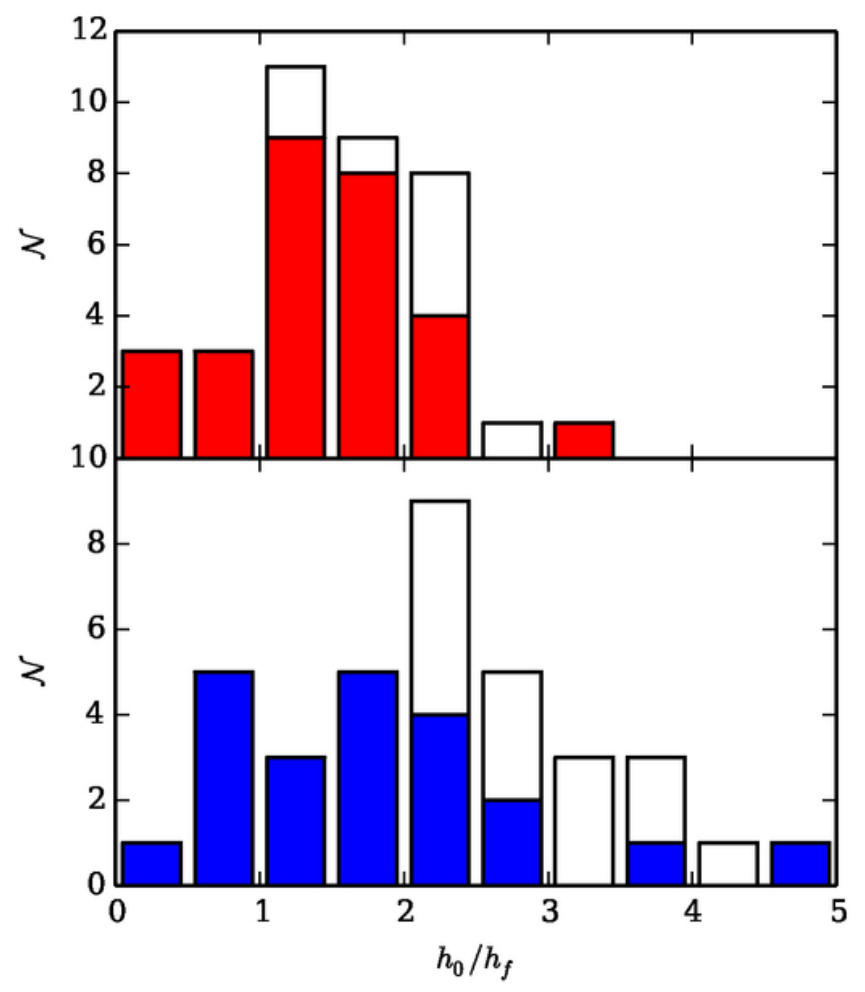

Figure 8. Histogram of $h_{0} / h_{f}$ for the final sample. In the top plot, the results are shown for the EP profile. Filled, red bars are the breaks. Stacked on top of that are the white bars for the truncations. The bottom panel features the results for the PAS profiles, in blue the breaks and in white the truncations.

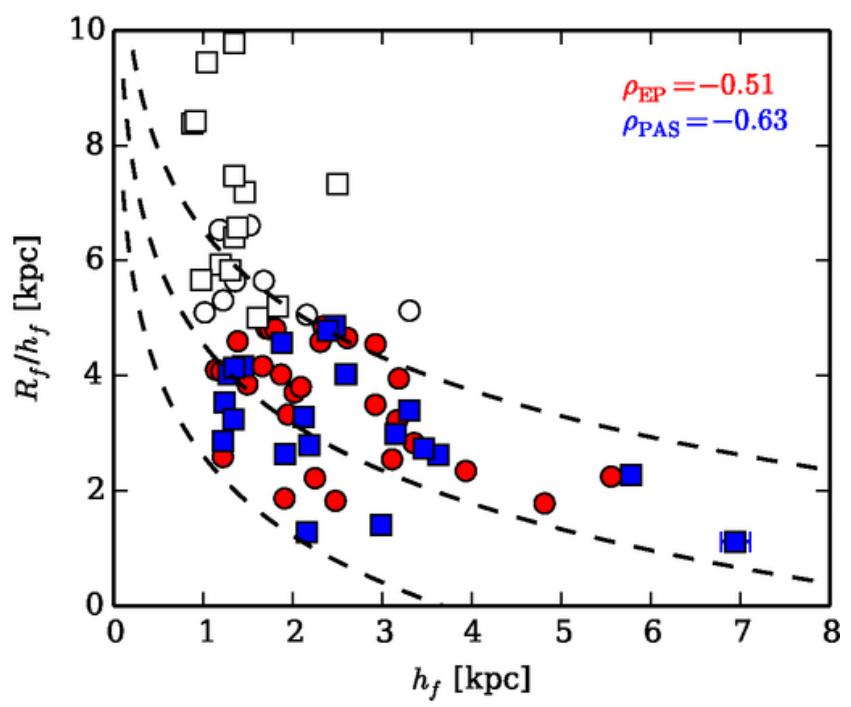

Figure 9. Scale length $h_{f}$ with ratio $R_{f} / h_{f}$. Filled markers are breaks, open markers are truncations. The top panel shows the results for the PAS and the bottom panel for the EP. Dashed black lines represent the empirical scaling relations from Schaye (2004), using $\Sigma_{0}=5.9 \mathrm{M}_{\odot} \mathrm{pc}^{2}$ and $M_{\text {disc }}$ as $5 \times 10^{8}, 3.5 \times 10^{9}$ and $25 \times 10^{9} \mathrm{M}_{\odot}$.

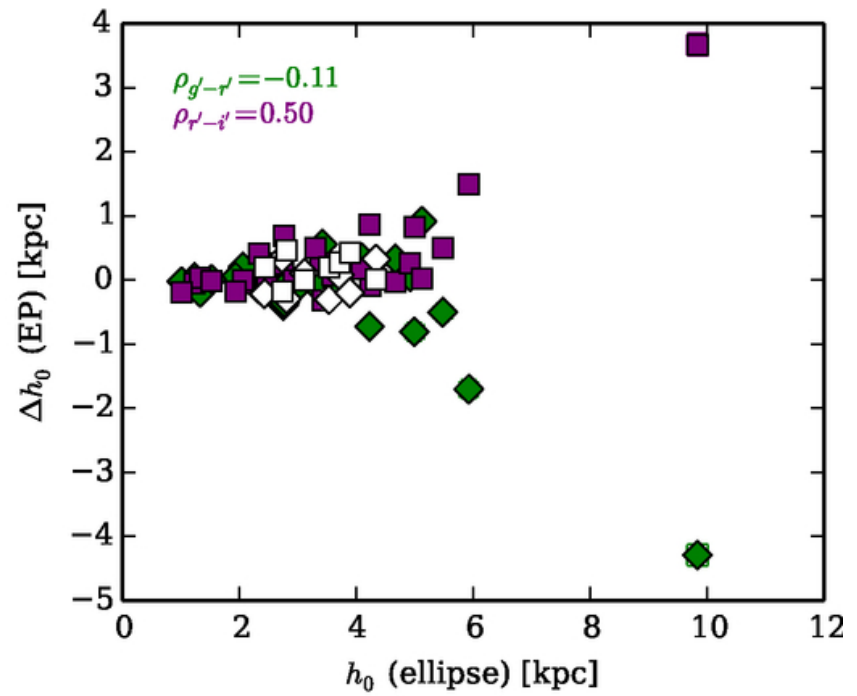

Figure 10. Correlations for differences in scale length $h_{0}$ per band as measured using the EP profiles, with the scale length from the r'-band ellipse-fit profiles. The corresponding significance values are superimposed on the panel. Diamond shaped boxes represent the $g^{\prime}-r^{\prime}$ points, square boxes represent the $r^{\prime}-i^{\prime}$. Filled markers are breaks, open markers are truncations.

after studying simulations of the thermal and ionization structure of the gaseous discs by Mo et al. (1998). The transition to the cold interstellar medium phase is responsible for the onset of local gravitational instability, which triggers star formation. For an exponential disc, an empirical relation was found which could match the data well (Equation 5). Here, $M_{\text {disc }}$ is the total mass of the disc an $\Sigma_{c}$ is the critical face-on surface density of the disc (Kregel \& van der Kruit 2004),

$$
\frac{R_{f}}{h_{f}}=\ln \frac{M_{\mathrm{disc}}}{2 \pi h_{f}^{2} \Sigma_{c}} .
$$

We apply this model to Figure 9 . The model fits the breaks sub-sample well, but fails to recover the general shape of the sample if we include the truncations sample.

\subsubsection{Variations per band}

It is interesting to test for variations of the scale lengths as measured in different bands. We test this by performing a correlation test of the ellipse-fit scale lengths $h_{0}$ and $h_{f}$ with the differences of the scale lengths measured in two different bands $\Delta h_{0}$ and $\Delta h_{f}$. So e.g. $\Delta h_{0}\left(g^{\prime}-r^{\prime}\right)(\mathrm{PAS})$ is the difference in scale length $h_{0}$ measured in $g^{\prime}$ and $r^{\prime}$ using the PAS method. The results from these tests are shown in Table 7 (lines 5 to 12). Only one statistically significant correlation is found. The difference of the inner scale lengths measured with the PAS in the $r^{\prime}$ and $i^{\prime}$ bands has a correlation of $\rho=0.50$ at $p<0.005$. We demonstrate this correlation in Figure 10 The points that lead to this correlation are mostly points with high uncertainty, and we thus remain skeptical about any actual correlation.

The differences in brightness between bands at the features from both methods are compared in Table 8 . If the error-bars are included in the comparison, the brightness 


\begin{tabular}{|c|c|c|c|c|c|c|c|}
\hline Variable 1 & Variable 2 & $\rho$ & $p$ & $\rho_{\text {break }}$ & $p_{\text {break }}$ & $\rho_{\text {truncation }}$ & $p_{\text {truncation }}$ \\
\hline$h_{0}(\mathrm{ell})$ & $h_{0}(\mathrm{EP})$ & 0.966 & $<0.01$ & 0.969 & $<0.01$ & 0.952 & $<0.01$ \\
\hline$h_{0}($ ell $)$ & $h_{0}(\mathrm{PAS})$ & 0.874 & $<0.01$ & 0.879 & $<0.01$ & 0.881 & $<0.01$ \\
\hline$h_{f}(\mathrm{ell})$ & $h_{f}(\mathrm{EP})$ & 0.942 & $<0.01$ & 0.925 & $<0.01$ & 0.952 & $<0.01$ \\
\hline$h_{f}(\mathrm{ell})$ & $h_{f}(\mathrm{PAS})$ & 0.875 & $<0.01$ & 0.927 & $<0.01$ & 0.732 & $<0.01$ \\
\hline$h_{0}(\mathrm{ell})$ & $\Delta h_{0}\left(g^{\prime}-r^{\prime}\right)(\mathrm{PAS})$ & -0.11 & 0.53 & -0.16 & 0.41 & 0.14 & 0.74 \\
\hline$h_{0}(\mathrm{ell})$ & $\Delta h_{0}\left(r^{\prime}-i^{\prime}\right)(\mathrm{PAS})$ & 0.496 & $<0.01$ & 0.537 & $<0.01$ & 0.24 & 0.57 \\
\hline$h_{0}($ ell $)$ & $\Delta h_{0}\left(g^{\prime}-r^{\prime}\right)(\mathrm{PAS})$ & 0.03 & 0.85 & 0.10 & 0.66 & $<0.01$ & 0.99 \\
\hline$h_{0}(\mathrm{ell})$ & $\Delta h_{0}\left(r^{\prime}-i^{\prime}\right)(\mathrm{PAS})$ & 0.03 & 0.86 & 0.06 & 0.80 & -0.07 & 0.81 \\
\hline$h_{f}(\mathrm{ell})$ & $\Delta h_{f}\left(g^{\prime}-r^{\prime}\right)(\mathrm{EP})$ & 0.22 & 0.20 & 0.19 & 0.33 & 0.33 & 0.42 \\
\hline$h_{f}(\mathrm{ell})$ & $\Delta h_{f}\left(r^{\prime}-i^{\prime}\right)(\mathrm{EP})$ & -0.31 & 0.07 & -0.20 & 0.32 & -0.38 & 0.35 \\
\hline$h_{f}(\mathrm{ell})$ & $\Delta h_{f}\left(g^{\prime}-r^{\prime}\right)(\mathrm{EP})$ & 0.23 & 0.18 & 0.40 & 0.06 & -0.18 & 0.53 \\
\hline$h_{f}(\mathrm{ell})$ & $\Delta h_{f}\left(r^{\prime}-i^{\prime}\right)(\mathrm{EP})$ & -0.15 & 0.38 & -0.18 & 0.43 & 0.29 & 0.31 \\
\hline$R_{f}(\mathrm{ell})$ & $R_{f}(\mathrm{EP})$ & 0.977 & $<0.01$ & 0.973 & $<0.01$ & 0.952 & $<0.01$ \\
\hline$R_{f}(\mathrm{ell})$ & $R_{f}(\mathrm{PAS})$ & 0.936 & $<0.01$ & 0.878 & $<0.01$ & 0.960 & $<0.01$ \\
\hline$\mu_{r^{\prime}}(\mathrm{EP})$ & $\mu_{g^{\prime}-r^{\prime}}(\mathrm{EP})$ & -0.07 & 0.71 & -0.15 & 0.46 & 0.29 & 0.49 \\
\hline$\mu_{r^{\prime}}(\mathrm{EP})$ & $\mu_{r^{\prime}-i^{\prime}}(\mathrm{EP})$ & -0.05 & 0.77 & -0.02 & 0.92 & -0.24 & 0.57 \\
\hline$\mu_{r^{\prime}}(\mathrm{PAS})$ & $\mu_{g^{\prime}-r^{\prime}}(\mathrm{PAS})$ & -0.26 & 0.13 & -0.34 & 0.13 & 0.17 & 0.55 \\
\hline$\mu_{r^{\prime}}(\mathrm{PAS})$ & $\mu_{r^{\prime}-i^{\prime}}(\mathrm{PAS})$ & 0.30 & 0.08 & 0.487 & 0.02 & -0.09 & 0.75 \\
\hline$\mu_{g^{\prime}-r^{\prime}}(\mathrm{EP})$ & $v_{\text {rot }}$ & 0.359 & 0.03 & 0.37 & 0.05 & 0.19 & 0.65 \\
\hline$\mu_{g^{\prime}-r^{\prime}}(\mathrm{PAS})$ & $v_{\text {rot }}$ & 0.19 & 0.25 & 0.27 & 0.23 & 0.13 & 0.65 \\
\hline$\mu_{g^{\prime}-r^{\prime}}(\mathrm{EP})$ & $M_{\mathrm{B}, \mathrm{abs}}$ & -0.14 & 0.43 & -0.13 & 0.52 & -0.21 & 0.61 \\
\hline$\mu_{g^{\prime}-r^{\prime}}(\mathrm{PAS})$ & $M_{\mathrm{B}, \mathrm{abs}}$ & -0.08 & 0.64 & -0.13 & 0.56 & -0.06 & 0.84 \\
\hline$\mu_{r^{\prime}}(\mathrm{EP})$ & $v_{\text {rot }}$ & -0.15 & 0.38 & -0.22 & 0.26 & 0.26 & 0.53 \\
\hline$\mu_{r^{\prime}}(\mathrm{PAS})$ & $v_{\text {rot }}$ & -0.31 & 0.07 & -0.38 & 0.08 & -0.13 & 0.67 \\
\hline$\mu_{r^{\prime}}(\mathrm{EP})$ & $M_{\mathrm{B}, \mathrm{abs}}$ & 0.09 & 0.58 & 0.12 & 0.55 & 0.33 & 0.42 \\
\hline$\mu_{r^{\prime}}(\mathrm{PAS})$ & $M_{\mathrm{B}, \mathrm{abs}}$ & 0.21 & 0.22 & 0.34 & 0.13 & 0.40 & 0.15 \\
\hline$R_{f}(\mathrm{EP})$ & $v_{\text {rot }}$ & 0.03 & 0.87 & -0.04 & 0.84 & 0.36 & 0.39 \\
\hline$R_{f}(\mathrm{PAS})$ & $v_{\text {rot }}$ & 0.04 & 0.82 & -0.02 & 0.93 & 0.34 & 0.24 \\
\hline$R_{f}(\mathrm{EP})$ & $M_{\mathrm{B}, \mathrm{abs}}$ & -0.19 & 0.26 & -0.12 & 0.53 & -0.10 & 0.82 \\
\hline$R_{f}(\mathrm{PAS})$ & $M_{\mathrm{B}, \mathrm{abs}}$ & -0.17 & 0.32 & -0.12 & 0.59 & -0.10 & 0.74 \\
\hline$R_{f}(\mathrm{EP})$ & $j$ & 0.03 & 0.87 & -0.04 & 0.84 & 0.36 & 0.39 \\
\hline$R_{f}(\mathrm{PAS})$ & $j$ & 0.04 & 0.82 & -0.02 & 0.93 & 0.34 & 0.24 \\
\hline$a / b$ & $h_{0}(\mathrm{EP})$ & 0.06 & 0.75 & 0.14 & 0.48 & -0.44 & 0.27 \\
\hline$a / b$ & $h_{0}(\mathrm{PAS})$ & 0.07 & 0.70 & 0.26 & 0.25 & -0.34 & 0.23 \\
\hline$a / b$ & $h_{f}(\mathrm{EP})$ & -0.26 & 0.12 & -0.23 & 0.24 & -0.16 & 0.71 \\
\hline$a / b$ & $h_{f}(\mathrm{PAS})$ & -0.404 & 0.01 & -0.22 & 0.33 & -0.49 & 0.08 \\
\hline$a / b$ & $R_{f} / h_{f}(\mathrm{EP})$ & 0.30 & 0.08 & 0.35 & 0.07 & 0.31 & 0.45 \\
\hline$a / b$ & $R_{f} / h_{f}(\mathrm{PAS})$ & 0.349 & 0.04 & 0.37 & 0.09 & 0.36 & 0.20 \\
\hline$a / b$ & $h_{0} / h_{f}(\mathrm{EP})$ & 0.22 & 0.20 & 0.28 & 0.15 & -0.01 & 0.98 \\
\hline$a / b$ & $h_{0} / h_{f}(\mathrm{PAS})$ & 0.32 & 0.06 & 0.35 & 0.11 & 0.06 & 0.85 \\
\hline$R_{f}(\mathrm{EP})$ & $\mu_{g^{\prime}-r^{\prime}}(\mathrm{EP})$ & 0.10 & 0.57 & -0.01 & 0.97 & 0.62 & 0.10 \\
\hline$R_{f}(\mathrm{EP})$ & $\mu_{r^{\prime}-i^{\prime}}(\mathrm{EP})$ & 0.21 & 0.23 & 0.30 & 0.13 & -0.12 & 0.78 \\
\hline$R_{f}(\mathrm{PAS})$ & $\mu_{g^{\prime}-r^{\prime}}(\mathrm{PAS})$ & -0.349 & 0.04 & -0.35 & 0.11 & 0.09 & 0.75 \\
\hline$R_{f}(\mathrm{PAS})$ & $\mu_{r^{\prime}-i^{\prime}}(\mathrm{PAS})$ & 0.05 & 0.79 & 0.20 & 0.37 & -0.10 & 0.73 \\
\hline$h_{f}(\mathrm{EP})$ & $R_{f} / h_{f}(\mathrm{EP})$ & -0.515 & $<0.01$ & -0.449 & 0.02 & -0.17 & 0.69 \\
\hline$h_{f}(\mathrm{PAS})$ & $R_{f} / h_{f}(\mathrm{PAS})$ & -0.632 & $<0.01$ & -0.441 & 0.04 & -0.37 & 0.19 \\
\hline
\end{tabular}

Table 7. Correlation tests for the various variables. Most are self-explanatory, except possibly $j$, which is the specific angular momentum (see text). The $\rho$ stands for the Spearman correlation coefficient. The null-hypothesis is tested with $p$, where values of $p<0.05$ indicate a (2-sigma) significant correlation. These have been highlighted as boldface in the table.

\begin{tabular}{cccc} 
band \& method & full sample & breaks & truncations \\
\hline$\Delta \mu\left(g^{\prime}-r^{\prime}\right)(\mathrm{EP})$ & $0.38 \pm 0.14$ & $0.38 \pm 0.15$ & $0.39 \pm 0.09$ \\
$\Delta \mu\left(g^{\prime}-r^{\prime}\right)(\mathrm{PAS})$ & $0.41 \pm 0.29$ & $0.46 \pm 0.31$ & $0.32 \pm 0.23$ \\
$\Delta \mu\left(r^{\prime}-i^{\prime}\right)(\mathrm{EP})$ & $0.25 \pm 0.18$ & $0.27 \pm 0.19$ & $0.19 \pm 0.13$ \\
$\Delta \mu\left(r^{\prime}-i^{\prime}\right)(\mathrm{PAS})$ & $0.29 \pm 0.34$ & $0.28 \pm 0.39$ & $0.30 \pm 0.20$ \\
\hline
\end{tabular}

Table 8. Average brightness differences at the feature radius between various bands 


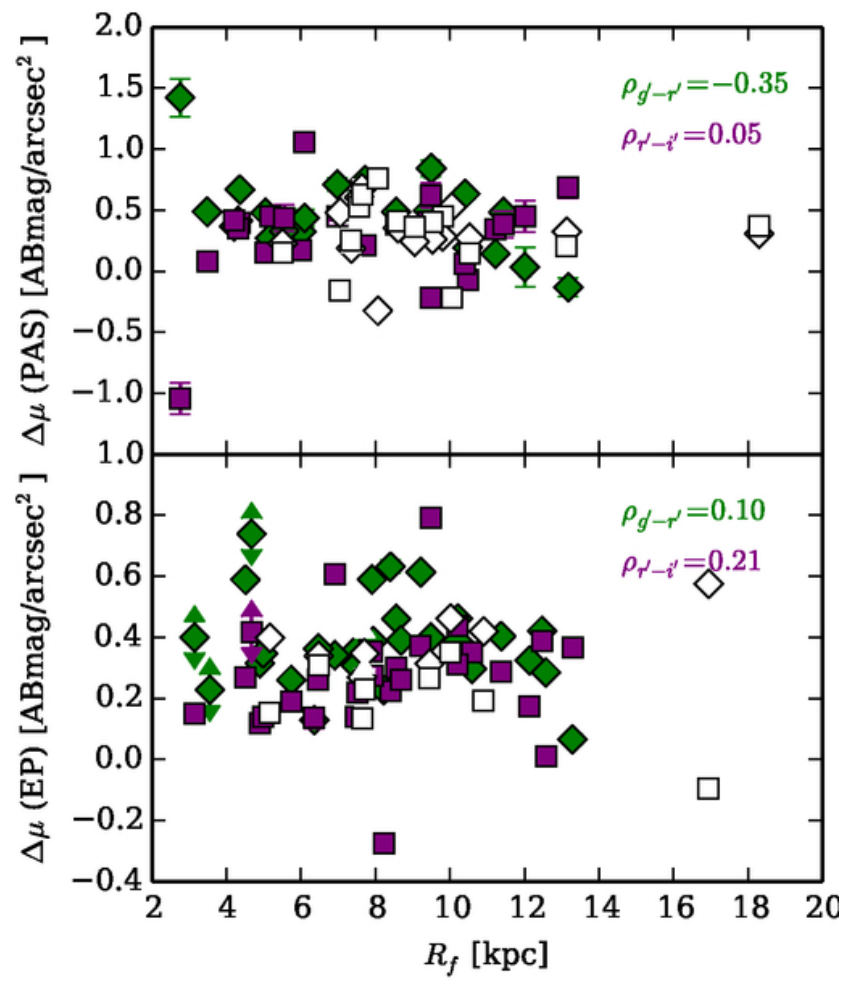

Figure 11. Correlations of the break radius $R_{f}$, for each method, with the difference in brightness for various bands. Shown are $g^{\prime}-r^{\prime}$ (diamond markers) and $r^{\prime}-i^{\prime}$ (box markers). Filled markers are breaks, open markers are truncations. The top panel shows the results for the PAS and the bottom panel for the EP.

differences between bands are practically the same, regardless of the use of either the EP or PAS. The one potential exception to this can be seen in the $r^{\prime}-i^{\prime}$ truncations sample, where for the EP we have $0.19 \pm 0.13$ and for the PAS $0.30 \pm 0.20$. As demonstrated earlier, the EP is particularly sensitive to background noise. Out of all bands, the $i^{\prime}$ has the highest background noise, so most likely the offset is due to sampling of the background. We have also tested any potential correlation of feature radius $r_{f}$ as measure by the EP or PAS profiles, with the brightness differences $g^{\prime}-r^{\prime}$ and $r^{\prime}-i^{\prime}$. We find a weak correlation of $\rho=-0.35$ and significance $p=0.04$ in the PAS $g^{\prime}-r^{\prime}$ full-dataset sample, which we show in Figure 11. This correlation is likely due to measurements errors of a couple of bad points at the outskirts.

The r'-band magnitude at the feature has also been compared to the $\Delta \mu\left(r^{\prime}-g^{\prime}\right)$ and $\Delta \mu\left(r^{\prime}-i\right)^{\prime}$ values. The rank correlation tests show that there is no strong correlation for $r^{\prime}$ with $\Delta \mu\left(r^{\prime}-g^{\prime}\right)$ and $\Delta \mu\left(r^{\prime}-i^{\prime}\right)$, with the maximum at only $\rho=0.30$ and $p=0.08$. The only exception is for the PAS profiles from the $r^{\prime}-i^{\prime}$ breaks subsample, where there is a correlation of $\rho=0.49$ and $p=0.02$. This correlation is due to the same points as the correlation of $h_{0}$ with $\Delta h_{0}\left(r^{\prime}-i^{\prime} 0\right)$ in the PAS.

\subsubsection{Correlations with absolute magnitude, maximum rotation and specific angular momentum}

Martín - Navarro et al. (2012) found that the truncation radius of a galaxy is strongly correlated with the maximum rotational velocity $v_{\text {rot }}$ of a galaxy, having a correlation of $\rho_{\text {truncation }}=0.81$. The breaks in their sample were correlated at only $\rho_{\text {break }}=0.50$. In contrast, Pohlen \& Trujillo (2006) report finding no correlation with rotation. They do report a weak correlation of the brightness at the feature $\mu_{r^{\prime}}$ with the absolute magnitude $M_{\mathrm{B} \text {,abs }}$. We explore possible correlations with of the feature radii $r_{f}$, surface brightness at the feature $\mu$, and differences in brightness between bands at the feature $\mu \Delta$, with absolute magnitude $M_{\text {abs }}$, maximum rotation $v_{\text {rot }}$, in Table 7 . We find only one weak correlation in the difference between the $g^{\prime}$ and $r^{\prime}$ surface brightness at the feature radius, as measured with the EP method, with the rotation velocity $v_{\text {rot }}$. The correlation strength is $\rho=0.36$ and $p=0.03$.

Martín-Navarro et al. (2012) also perform a correlation test of the break and truncation radii with the specific angular momentum $j$, calculated using the empirical expression by Navarro \& Steinmetz (2000

$$
j \approx 1.3 \times 10^{3}\left[\frac{v_{\text {rot }}}{200 \mathrm{~km} \mathrm{~s}^{-1}}\right]^{2} \mathrm{~km} \mathrm{~s}^{-1} \mathrm{~h}^{-1} \mathrm{kpc} .
$$

As this is a rescaling of $v_{\text {rot }}$, the correlation remains the same. They find that the feature radius only correlates well beyond $r_{\mathrm{b}}=8 \mathrm{kpc}$. For smaller disc with $v_{\text {rot }}<100 \mathrm{~km} \mathrm{~s}^{-1}$, $r_{\text {break }}$ and $v_{\text {rot }}$ are essentially unlinked. When examining the full radii range, we also do not find a statistically significant correlation. The same holds when limiting ourselves to all features beyond $r>8$.

\subsubsection{Effect of inclination}

In Section 4.4.2, we found that the PAS profiles typically tends to have sharper breaks than the ellipse-fit and EP profile. As the PAS projects data as if it was edge-on, it is also interesting to compare the effect of inclinations in this. To this end, we have tested the correlation of $a / b$ (major axis over minor axis) with scale lengths $h_{0}$ and $h_{f}, h_{0} / h_{f}$ and $R_{f} / h_{f}$, in Table 7 . The results are also visualized in Figure 12

We find no significant correlation between $a / b$ and inner scale length $h_{0}$. There may be a negative trend visible in the truncations-only sample, as both the EP and PAS profiles have reasonably strong correlations with $\rho=-0.44$ and $\rho=-0.34$, but more point will be required before this is significant. When looking at the outer scale length, a negative correlation of $\rho=-0.40$ at $p=0.01$ is found for the PAS. This correlation is mostly due to the truncations samples, which has $\rho=-0.49$ at $p=0.08$, which is also clear from the figure. Thus, the PAS outer scale length typically gets shorter with higher inclination, while the inner scale lengths barely depend on inclination.

The $R_{f} / h_{f}$ has a weak but significant correlation with $a / b$ in the PAS method, $\rho=0.35$ at $p=0.04$, a correlation which holds (albeit insignificantly) for both subsets. Interestingly the EP results follow similar correlations, although 

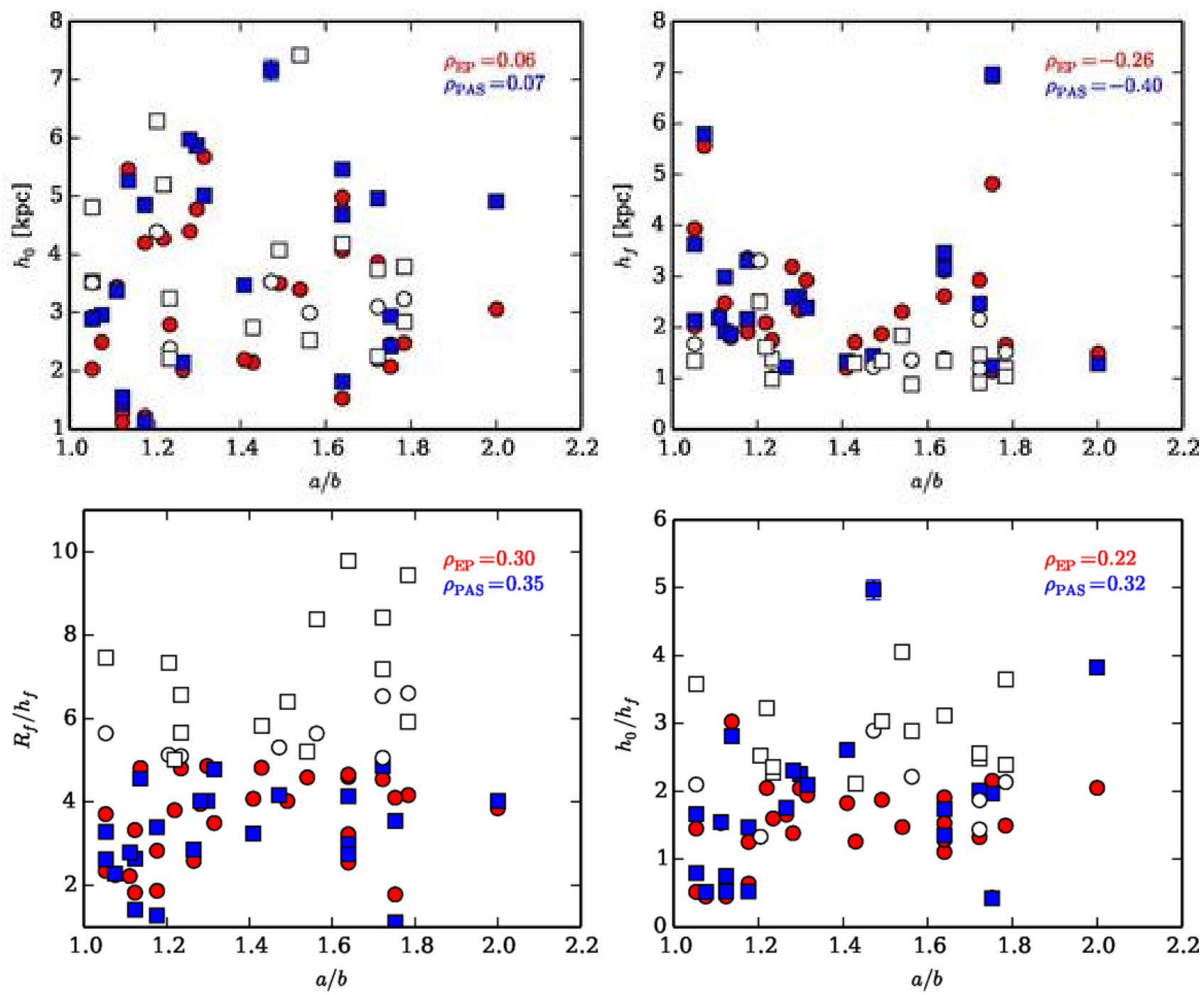

Figure 12. Correlation of various parameters with inclination (expressed as $a / b$ ). Top row shows with scale length $h_{0}\left(\right.$ left) and $h_{f}$ (right). Bottom row has the $h_{0} / h_{f}$ (right) and $R_{f} / h_{f}$ (left) correlations. The statistical correlation tests are plotted in each panel. Boxes represent the PAS points, while circles represent the EP points. Open markers represent truncations, while filled markers represent boxes.

those are not significant. The sharpness of the breaks $h_{0} / h_{f}$ has no significant correlation with $a / b$ in either method.

\section{CLOSING DISCUSSION AND CONCLUSIONS}

We have developed two new approaches for extracting the surface photometry of a face-on galaxy. The Equivalent Profiles (EP) work under the assumption that the surface brightness of a galaxy decreases as the radius increases. By starting with the brightest pixel and moving to lower brightness levels, each level can be assigned an equivalent area ellipse containing the surface of all pixels that are at or brighter than that level. The equivalent ellipse then gives the equivalent radius. The other method is the Principle Axis Summation (PAS), which work by summing the light onto the principle axis of the galaxy. This method then gives the equivalent of the profile as if the galaxy was seen edge- on. We have then tested this method on a sub-sample of the galaxies from Pohlen \& Trujillo (2006).

Seen overall, we find that both our methods perform well. Considering the fundamentally different method used to derive them, a detailed comparison that we have made (not illustrated) shows to us that the EP are remarkably similar to the ellipse-fit profiles as measured by Pohlen \& Trujillo (2006). We also point out that the classical method of elliptical averaging compares very well with results of equivalent profiles, (van der Kruit 1979). There are some differences. The ellipse-fit profiles have the ability to measure local upturns in the profiles, for example due to a local bar or ring feature. By design, the EP is unable to cope with this. This can lead to slightly different scale lengths. Beyond such a bump however, the EP profile and ellipse-fit join up again, as for example in Figure A1. Overall, we see that the EP behaves worse at lower brightness levels than the ellipse-fit profiles. For practical purposes, the ellipse therefore remains the preferred method.

The PAS method turns out to be a very interesting 
approach. Compared to the EP profiles, breaks and truncations often look sharper. A good example of this can be seen in galaxy IC1158, seen in Figure A3, where the PAS profiles starts to drop quite rapidly beyond $\sim 65^{\prime \prime}$, much stronger than the ellipse-fit profiles. We find that the inner scale length as measured with the PAS is on average $10 \%$ longer than the same scale length in either of the other methods. We also find a negative correlation with the inclination as expressed by the ratio $a / b$. As the inclination increase, the outer scale length of the PAS profiles get smaller. This leads to sharper breaks $h_{0} / h_{f}$ than is seen in ellipse-fit profiles or in the EP profiles. Although beyond the scope of this project, it would be interesting to test if $h_{0} / h_{f}$, rather than $R_{b} / h_{f}$ is a good way to distinguish between breaks and truncations.

In edge-on galaxies, there is a well-observed correlation of the radius of the truncations with the maximum rotation velocity $v_{\text {rot }}$ (van der Kruit 2008). This was confirmed by Martín - Navarro et al. (2012), who also found a correlation with the absolute magnitude of the galaxy $M_{\mathrm{B} \text {,abs }}$. Various studies of face-on samples, starting with Pohlen \& Trujillo (2006) and more recently for example (Muñoz-Mateos et al. 2013) have looked for and sometimes reported similar relations (e.g. their fig. 8), but in those cases it is not clear that the break radii used are referring to the equivalent features of edge-on truncations. We do not find any correlation of the surface brightness at the feature, difference in brightness between various bands at the feature, and the feature radius, with the absolute brightness $M_{\mathrm{B} \text {,abs }}$ nor with the maximum rotation $v_{\text {rot }}$. Martín-Navarro et al. (2012) divide their samples up into truncations and breaks based on the criteria $R_{f} / h_{0}=5$, with the galaxy belonging to breaks if the ratio was below five and truncations if it was above it. We have split our sample into these two subsets using the same criteria and have inspected the data for correlations. We do not reproduce these correlations. We are therefore skeptical of the galaxies in our truncations subsample constituting true truncations in the edge-on sense. It more likely that we are still only observing breaks. Truncations can likely only be found by using deeper imaging, such as that used by Bakos \& Trujillo (2012). We will explore the use of deeper imaging to detect truncations further in Peters et al. (2015).

\section{ACKNOWLEDGMENTS}

We thank Michael Pohlen for providing his data on the sample in electronic form.

SPCP is grateful to the Space Telescope Science Institute, Baltimore, USA, the Research School for Astronomy and Astrophysics, Australian National University, Canberra, Australia, and the Instituto de Astrofisica de Canarias, La Laguna, Tenerife, Spain, for hospitality and support during short and extended working visits in the course of his $\mathrm{PhD}$ thesis research. He thanks Roelof de Jong and Ron Allen for help and support during an earlier period as visiting student at Johns Hopkins University and the Physics and Astronomy Department, Krieger School of Arts and Sciences for this appointment.

PCK thanks the directors of these same institutions and his local hosts Ron Allen, Ken Freeman and Johan Knapen for hospitality and support during many work visits over the years, of which most were directly or indirectly related to the research presented in this series op papers.

Work visits by SPCP and PCK have been supported by an annual grant from the Faculty of Mathematics and Natural Sciences of the University of Groningen to PCK accompanying of his distinguished Jacobus C. Kapteyn professorhip and by the Leids Kerkhoven-Bosscha Fonds. PCK's work visits were also supported by an annual grant from the Area of Exact Sciences of the Netherlands Organisation for Scientific Research (NWO) in compensation for his membership of its Board.

\section{REFERENCES}

Abazajian K., Adelman-McCarthy J. K., Agüeros M. A., et al., 2004, AJ, 128, 502

Abazajian K. N., Adelman-McCarthy J. K., Agüeros M. A., et al., 2009, ApJS, 182, 543

Bakos J., Trujillo I., 2012, ArXiv e-prints

Bakos J., Trujillo I., Pohlen M., 2008, ApJl, 683, L103

Bosma A., Freeman K. C., 1993, AJ, 106, 1394

Busko I. C., 1996, in Jacoby G. H., Barnes J., eds, Astronomical Data Analysis Software and Systems V, Vol. 101 of Astr. Soc. Pacific Conf. Ser., . p. 139

Comerón S., Elmegreen B. G., Salo H., et al., 2012, ApJ, 759, 98

de Vaucouleurs G., 1948, Annales d'Astrophysique, 11, 247 de Vaucouleurs G., 1959, Handbuch der Physik, 53, 311

Elmegreen B. G., Hunter D. A., 2006, ApJ, 636, 712

Erwin P., Beckman J. E., Pohlen M., 2005, ApJl, 626, L81

Erwin P., Pohlen M., Beckman J. E., 2008, AJ, 135, 20

Freeman K. C., 1970, ApJ, 160, 811

Gutiérrez L., Erwin P., Aladro R., Beckman J. E., 2011, AJ, 142, 145

Herrmann K. A., Hunter D. A., Elmegreen B. G., 2013, AJ, 146,104

Herrmann K. A., Hunter D. A., Elmegreen B. G., 2016, astro-ph1606.00867

Jacob J. C., Katz D. S., Berriman G. B., et al.,, 2010, Montage: An Astronomical Image Mosaicking Toolkit

Jedrzejewski R. I., 1987, MNRAS, 226, 747

Jones C. K., Whittall K. P., MacKay A. L., 2003, Magnetic Resonance in Medicine, 50, 206

Knapen J. H., van der Kruit P. C., 1991, A\&A, 248, 57

Kregel M., van der Kruit P. C., 2004, MNRAS, 355, 143

Laine J., Laurikainen E., Salo H., et al., 2014, MNRAS, 441, 1992

Lang D., Hogg D. W., Mierle K., et al., 2010, AJ, 137, 1782

Martín - Navarro I., Bakos J., Trujillo I., et al., 2012, MNRAS, 427, 1102

Mo H. J., Mao S., White S. D. M., 1998, MNRAS, 295, 319

Muñoz-Mateos J. C., Sheth K., Gil de Paz A., et al. 2013, ApJ, 771, 59

Navarro J. F., Steinmetz M., 2000, ApJ, 538, 477

Patterson F. S., 1940, Harvard College Observatory Bulletin, 914, 9

Peters S. P. C., van der Kruit P. C., Knapen J. H., et al., 2015, MNRAS, submitted,

Pohlen M., Balcells M., Lütticke R., Dettmar R.-J., 2004, A\&A, 422, 465 
Pohlen M., Dettmar R.-J., Lütticke R., 2000, A\&A, 357, L1

Pohlen M., Dettmar R.-J., Lütticke R., Aronica G., 2002, A\&A, 392, 807

Pohlen M., Trujillo I., 2006, A\&A, 454, 759

Pohlen M., Zaroubi S., Peletier R., Dettmar R.-J., 2007, Mon.Not.Roy.Astron.Soc., 378, 594

Schaye J., 2004, ApJ, 609, 667

van der Kruit P. C., 1979, A\&AS, 38, 15

van der Kruit P. C., 1988, A\&A, 192, 117

van der Kruit P. C., 2008, in Funes J. G., Corsini E. M., eds, Formation and Evolution of Galaxy Disks Vol. 396 of Astr. Soc. Pacific Conf. Ser., . p. 173

van der Kruit P. C., Freeman K. C., 2011, ARA\&A, 49, 301

van der Kruit P. C., Searle L., 1981a, A\&A, 95, 105

van der Kruit P. C., Searle L., 1981b, A\&A, 95, 116

van der Kruit P. C., Searle L., 1982a, A\&A, 110, 61

van der Kruit P. C., Searle L., 1982b, A\&A, 110, 79

Wevers B. M. H. R., 1984, Ph.D. thesis, Univ. Groningen Wevers B. M. H. W., van der Kruit P. C., Allen R. J., 1984, A\&AS, 66, 505

\section{APPENDIX A: PLOTS FOR INDIVIDUAL GALAXIES}

The figures in this Appendix show the surface photometry for all galaxies in our sample.

The top-left image demonstrates the inner parts of the galaxy. The red ellipse denotes the trust radius $R_{\text {trust }}$ in which we have derived the PAS profiles. The white ellipse shows the area in which we have measured the EP profiles.

The top-right image shows an image of the deep background. The region between the two red ellipses (one and two times the trust radius $R_{\text {trust }}$ ) is used to estimate the background level. The inner ellipse is the same as the red one in the upper image.

The bottom panel shows all profiles for the $g^{\prime}, r^{\prime}$ and $i^{\prime}$ bands. The top set of curves are based on the PAS photometry. From top to bottom the set of curves is for the $i^{\prime}, r^{\prime}$ and $g^{\prime}$ bands. The horizontal axis in the profiles is in arcsec, the vertical one in magnitudes per arcsecond ${ }^{2}$. Note that the PAS has units of mag/arcsec. We have chosen the vertical offset such that the reader can easily compare with the other parameters. The bottom set of curves are from the EP photometry. The black curves are the $r^{\prime}$ ellipse-fit profiles from Pohlen \& Trujillo (2006). The vertical dashed lines represent the outermost radii for the PAS and EP ellipses. The horizontal dashed lines show the one-sigma noise levels of the background.
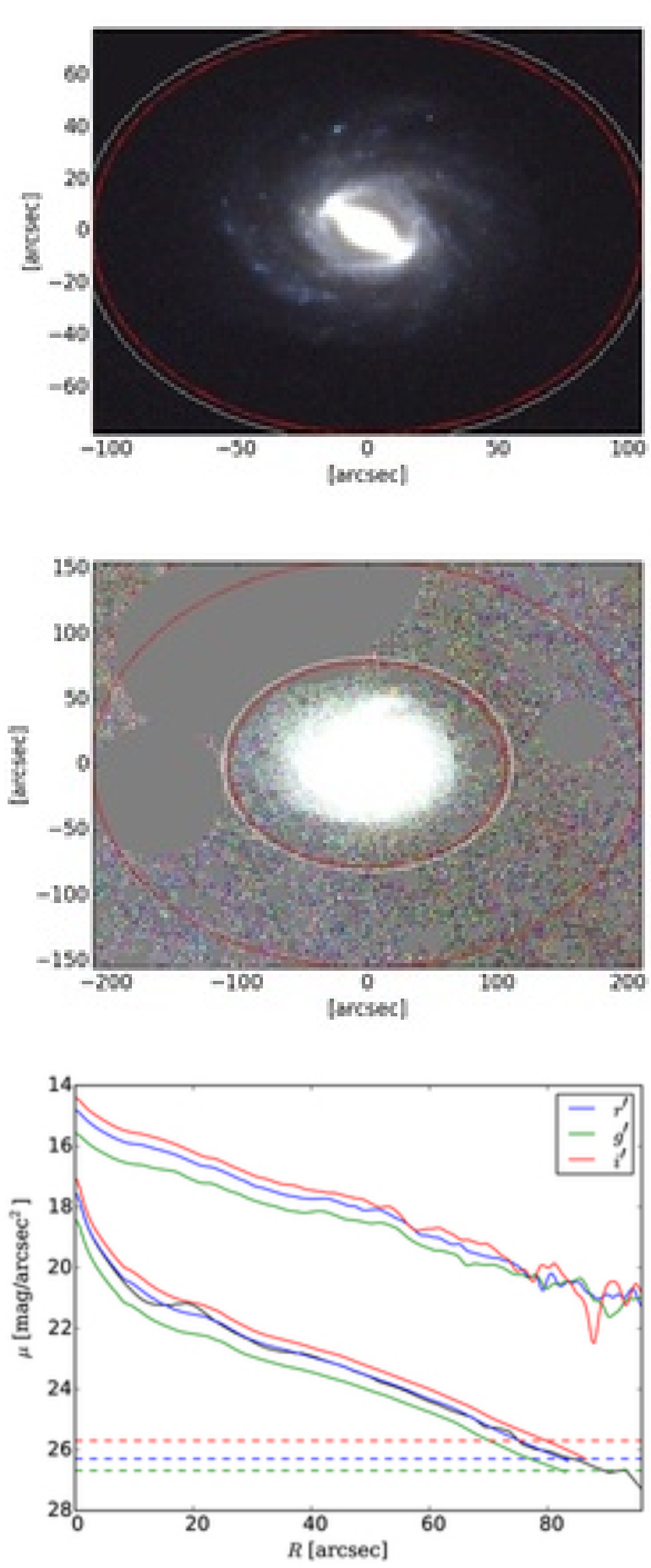

Figure A1. IC 1067: PAS is using the PAS is using the lower right and upper right quadrants. 

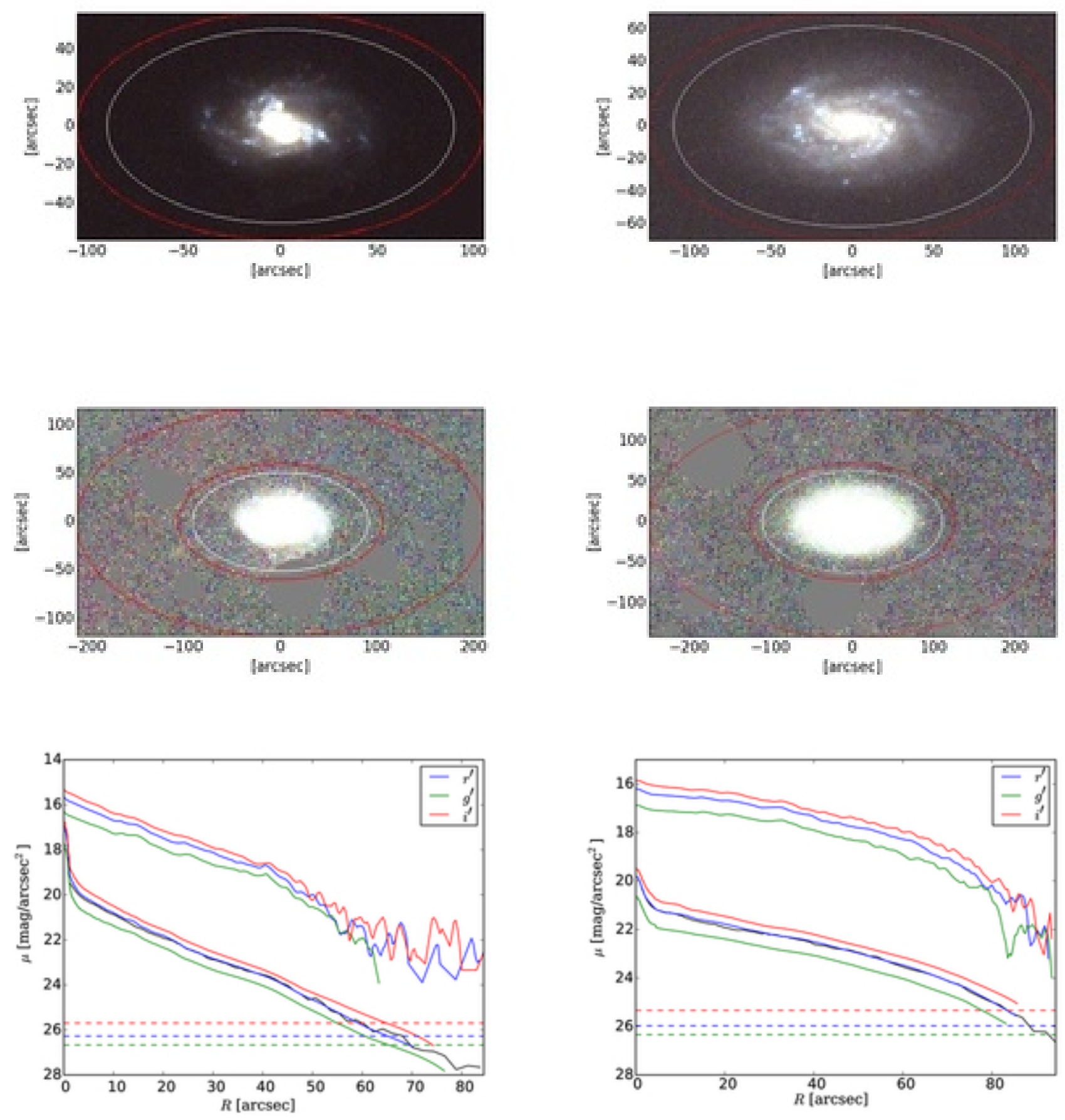

Figure A2. IC 1125: PAS is using all quadrants.

Figure A3. IC 1158: PAS is using all quadrants. 

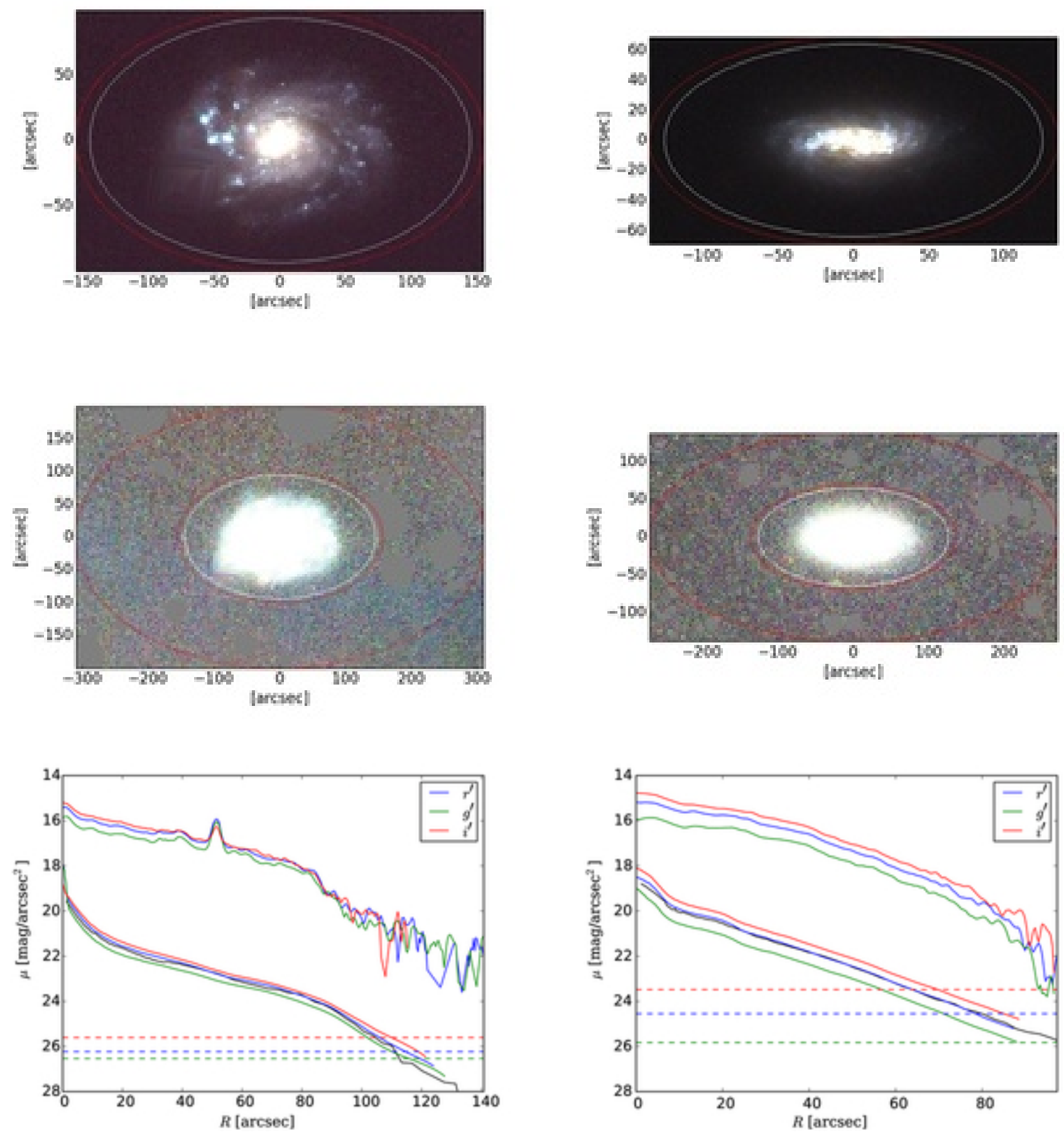

Figure A4. NGC 0450: PAS is using the lower left, lower right and upper right quadrants.

Figure A5. NGC 0701: PAS is using all quadrants. 

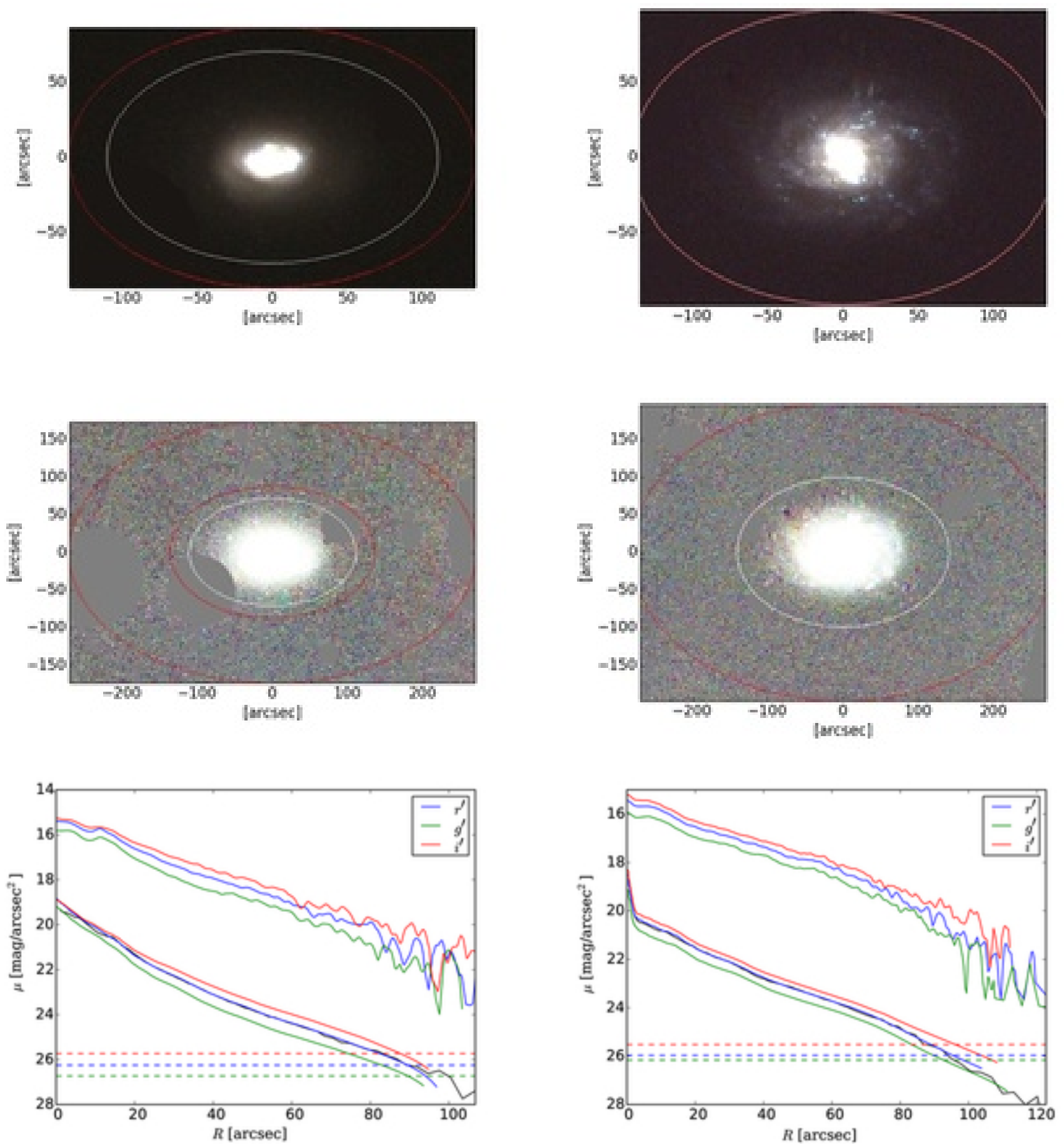

Figure A6. NGC 0853: PAS is using the PAS is using the lower left and upper right quadrants.

Figure A7. NGC 0941: PAS is using all quadrants. 

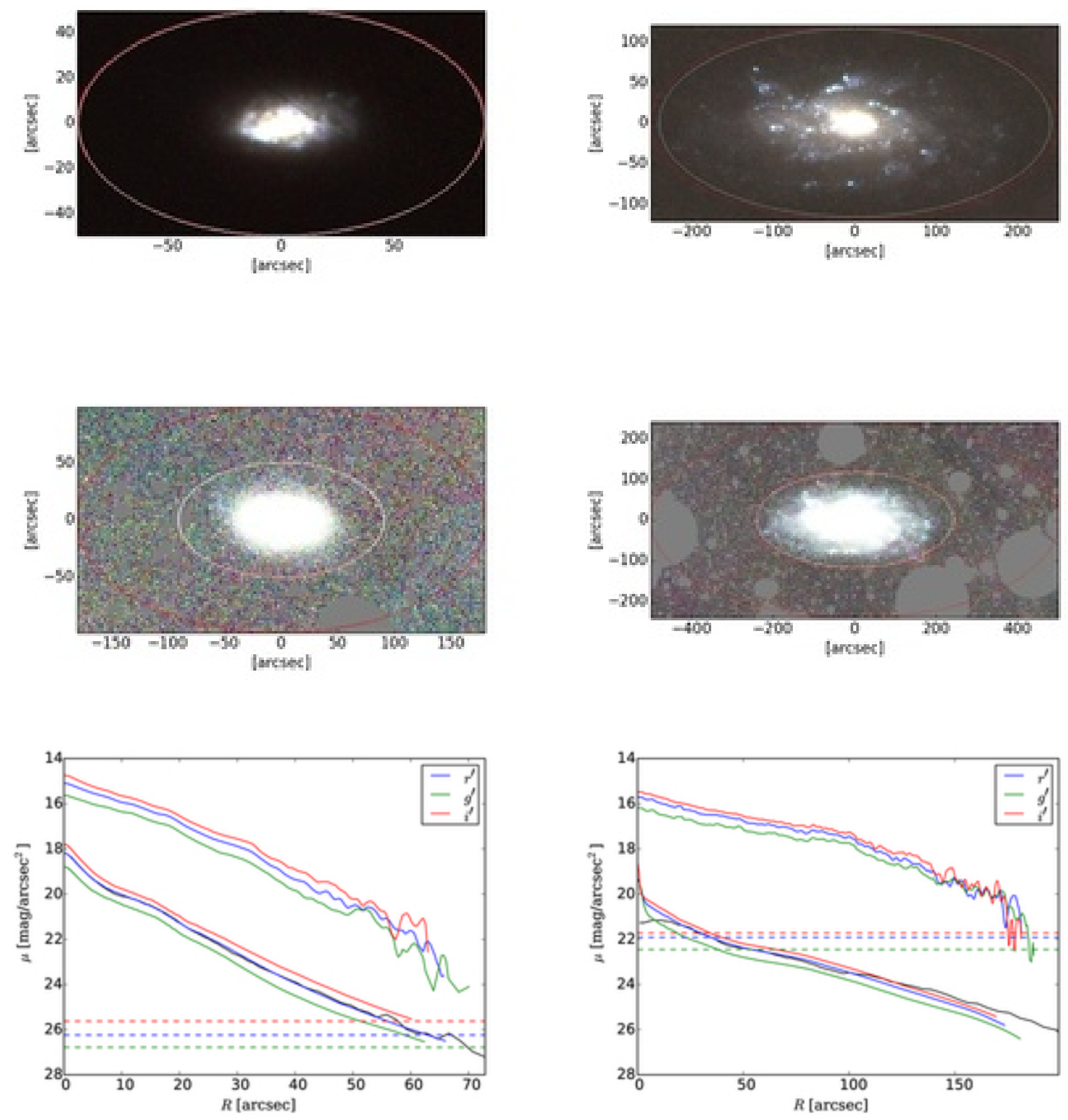

Figure A8. NGC 1299: PAS is using all quadrants.

Figure A9. NGC 2541: is not using the lower right quadrant. 

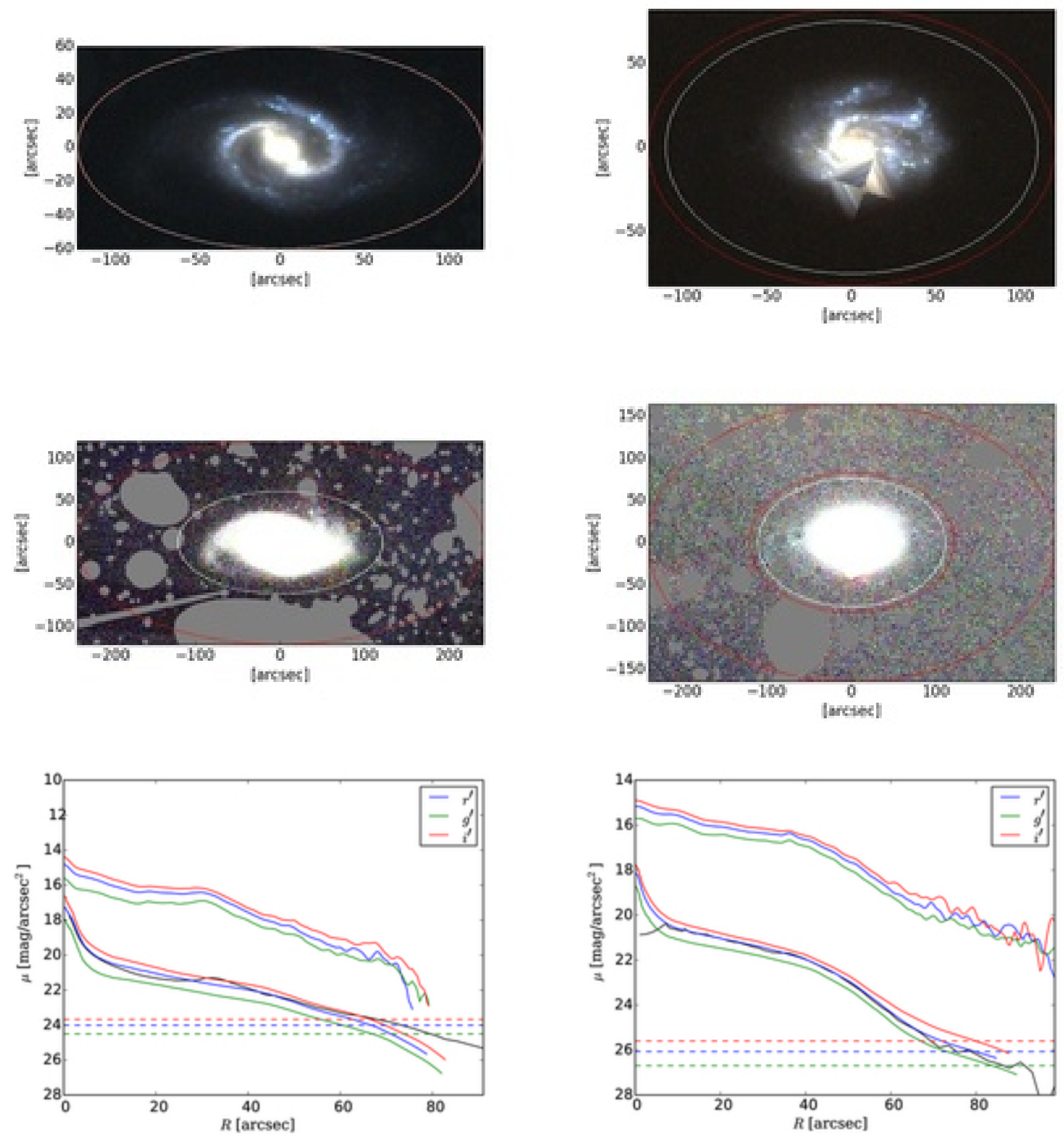

Figure A10. NGC 2543: PAS is using all quadrants.

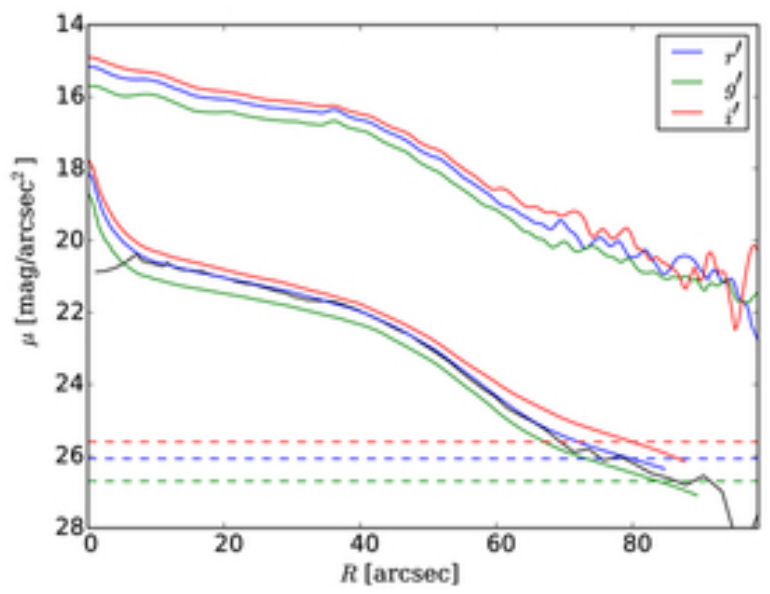

Figure A11. NGC 2701: PAS is using the PAS is using the lower left and lower right quadrants. 

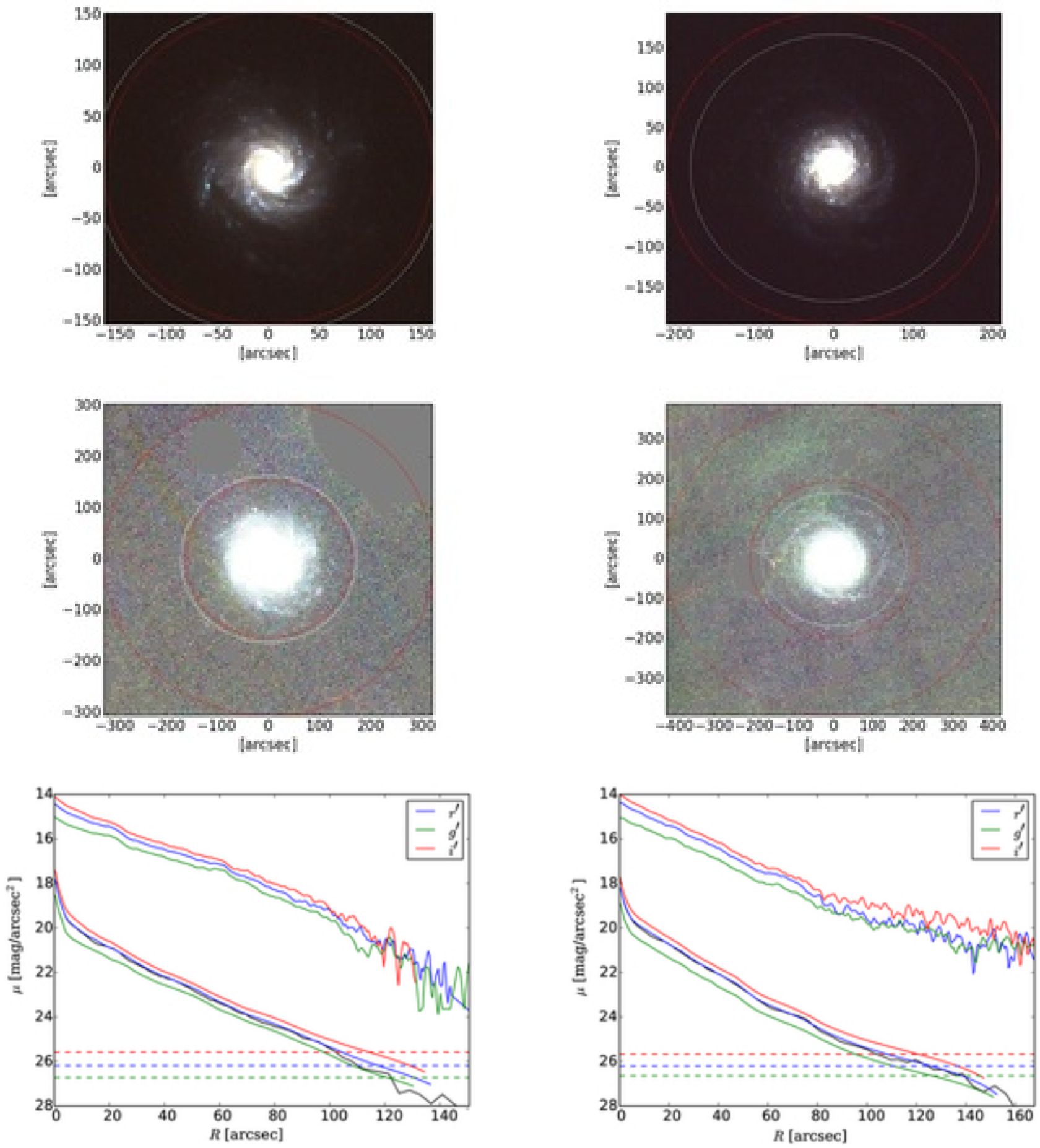

Figure A12. NGC 2776: PAS is using all quadrants.

Figure A13. NGC 2967: PAS is using all quadrants. 

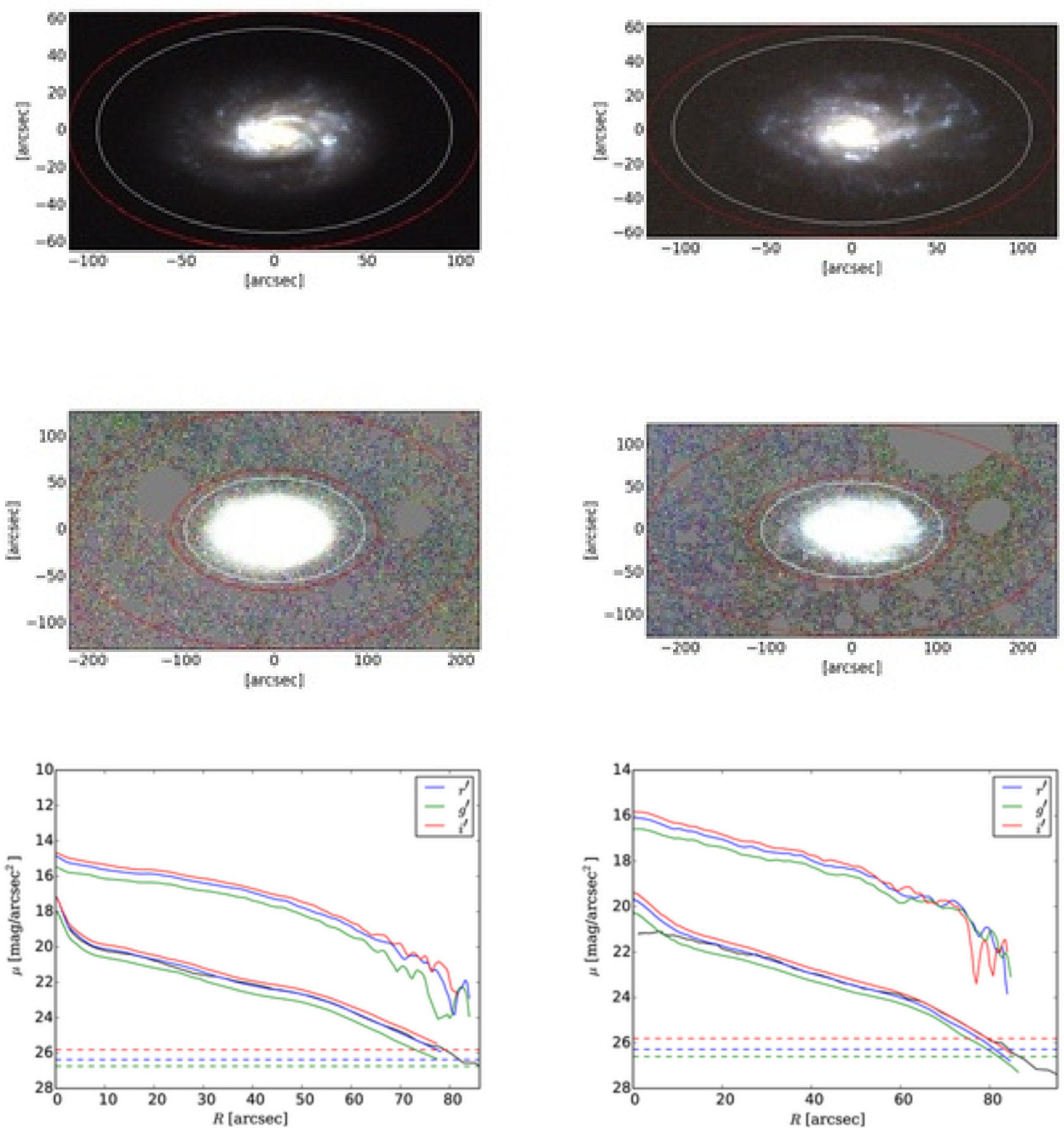

Figure A14. NGC 3055: PAS is using all quadrants.

Figure A15. NGC 3246: PAS is using all quadrants. 

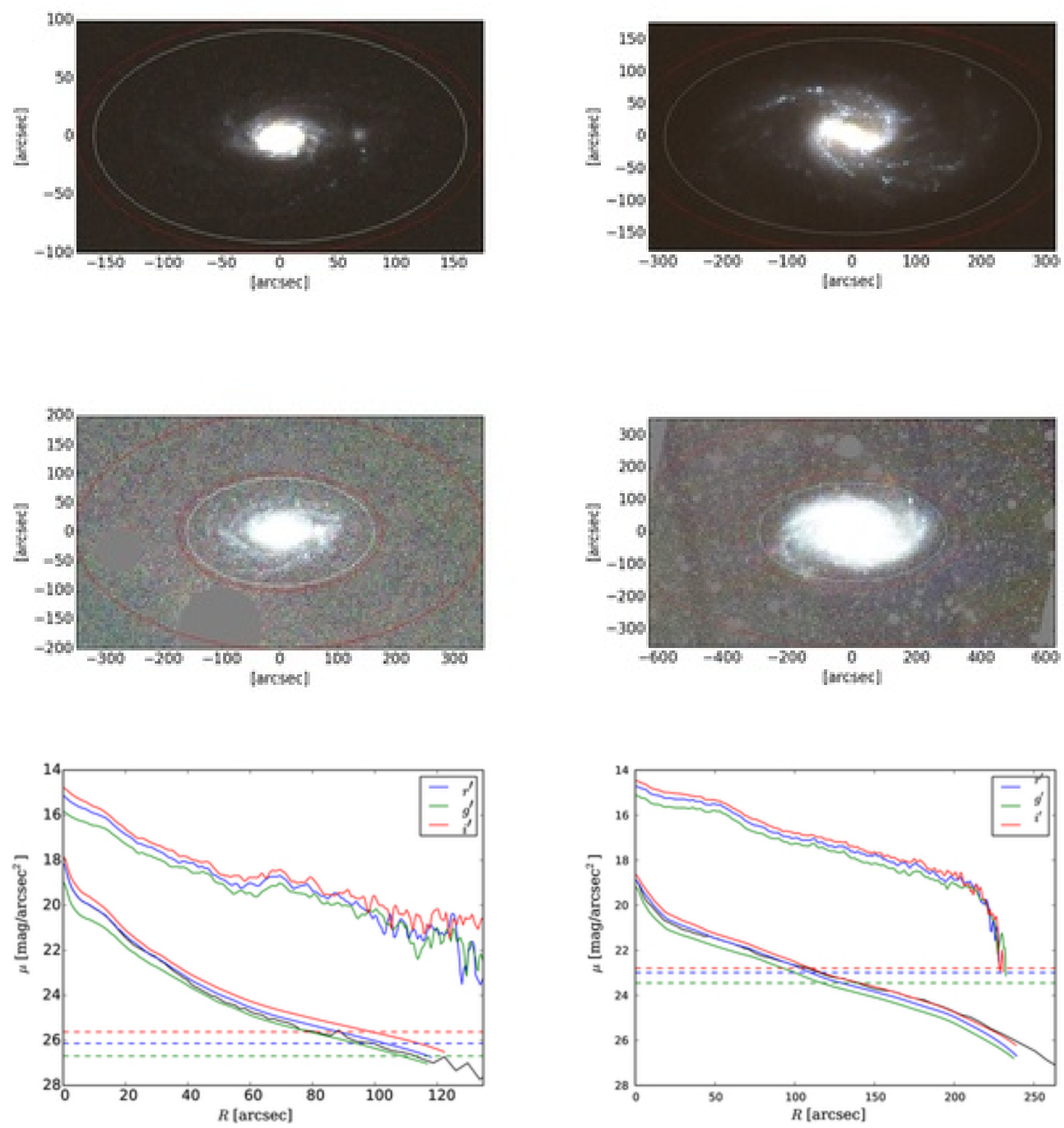

Figure A16. NGC 3259: PAS is using all quadrants.

Figure A17. NGC 3359: PAS is using all quadrants. 

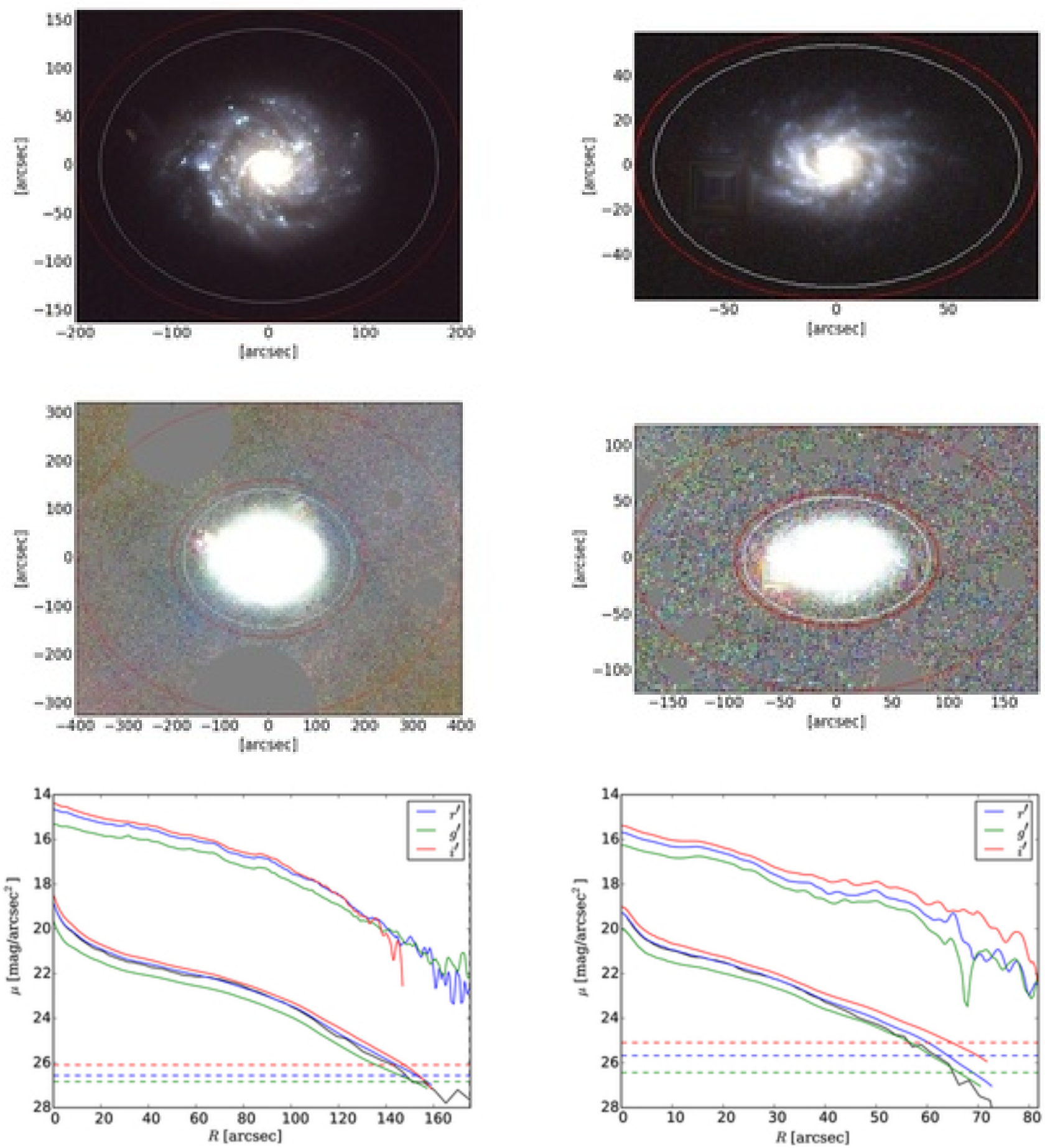

Figure A18. NGC 3423: PAS is using the PAS is using all quadrants.

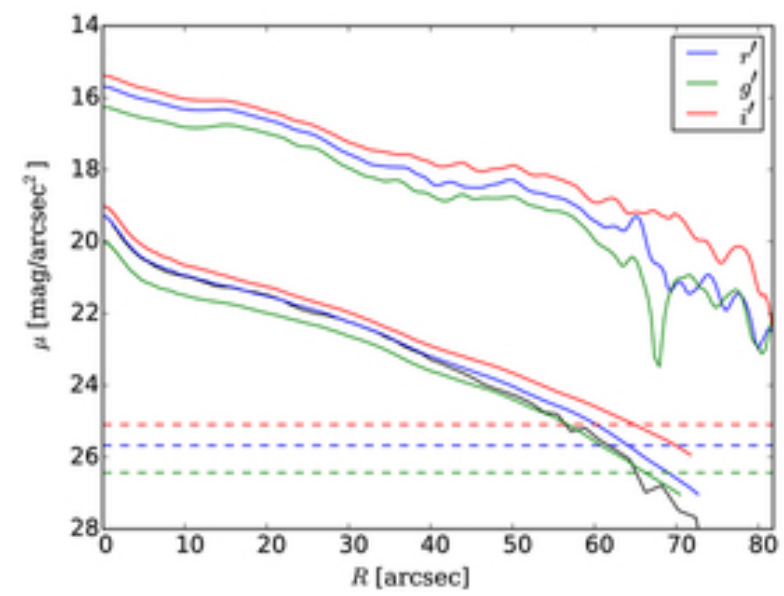

Figure A19. NGC 3488: PAS is using the PAS is using the lower right and upper right quadrants. 

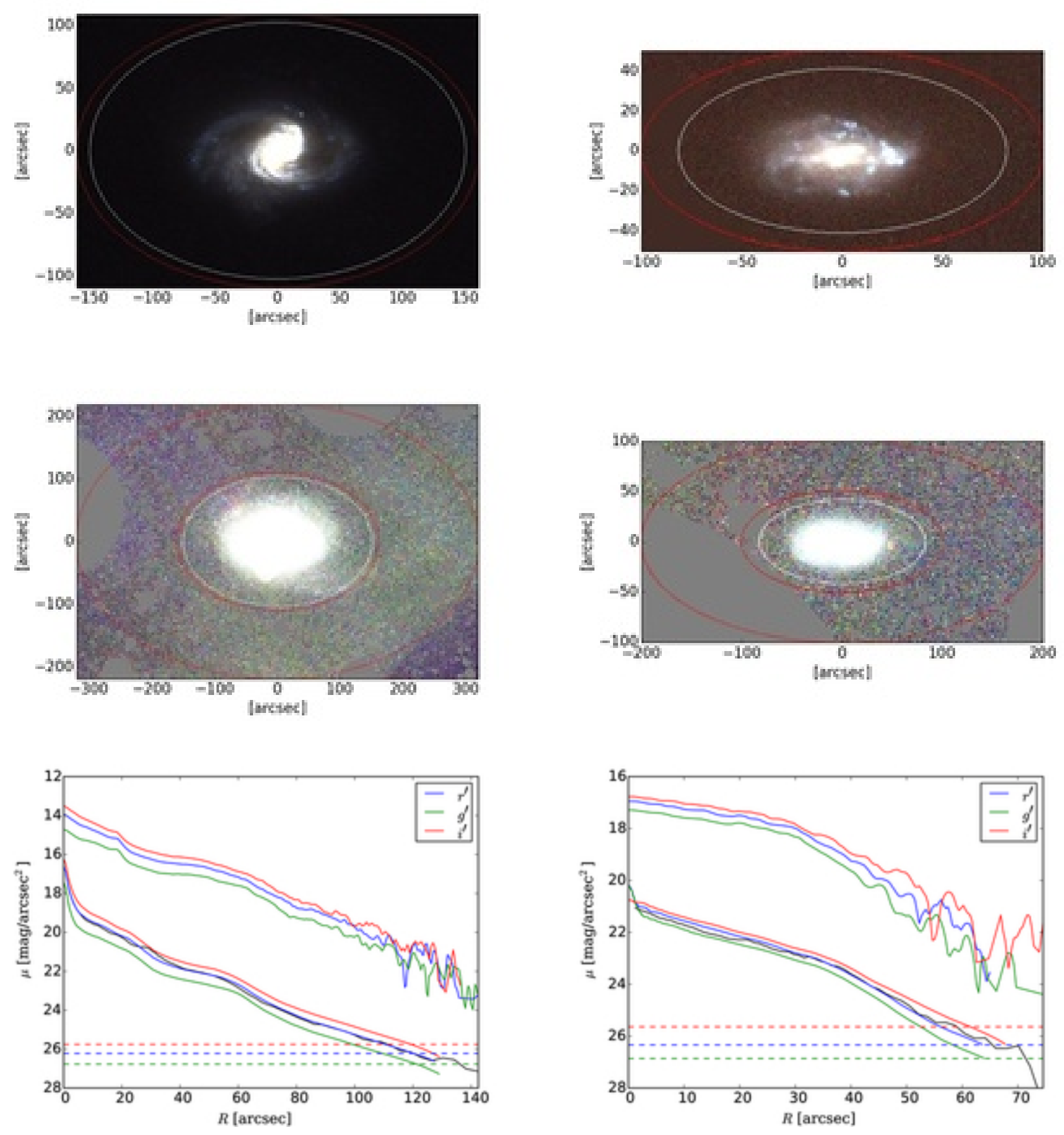

Figure A20. NGC 3583: PAS is using all quadrants.

Figure A21. NGC 3589: PAS is using all quadrants. 

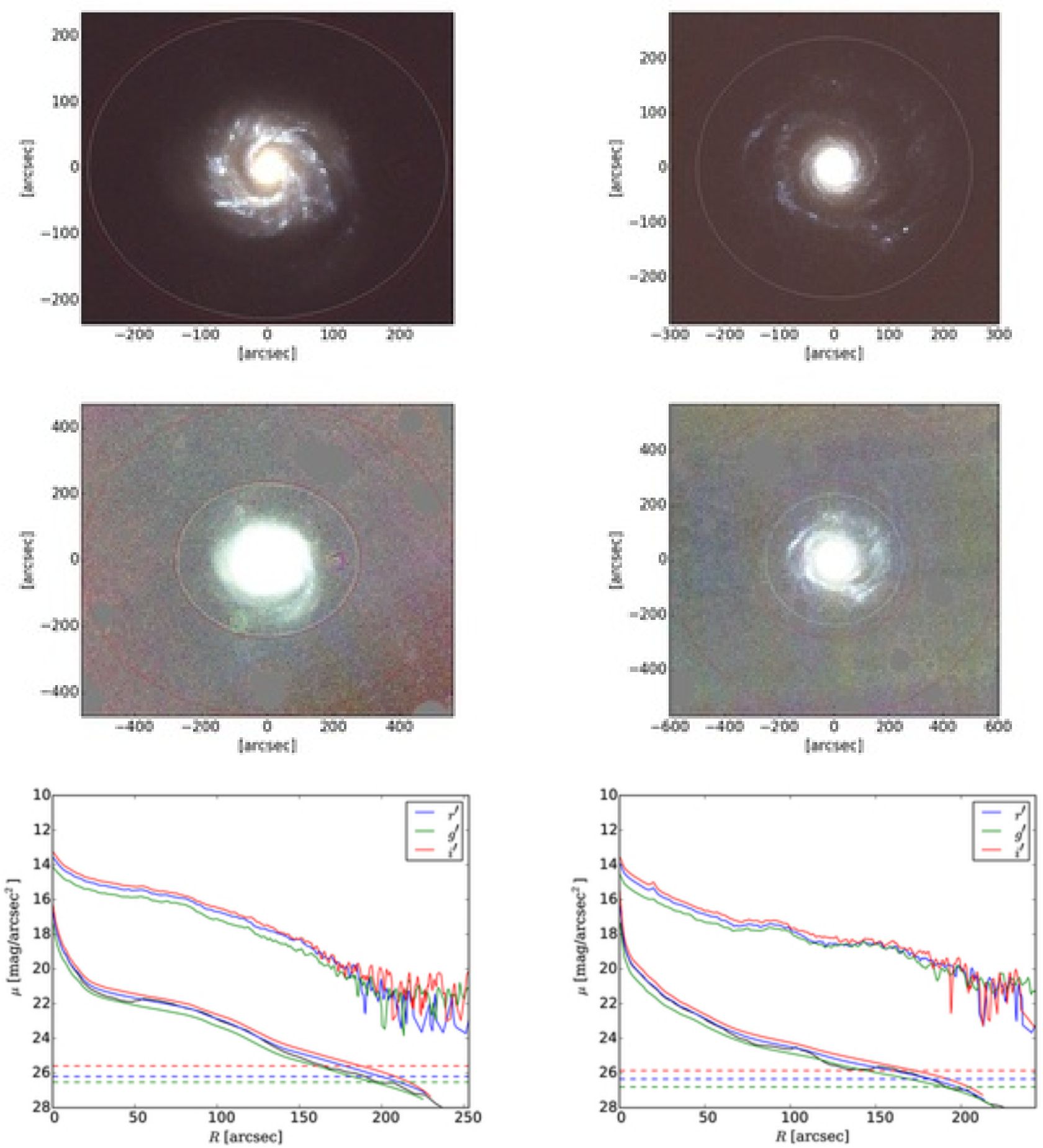

Figure A22. NGC 3631: PAS is using all quadrants.

Figure A23. NGC 3642: PAS is using all quadrants. 

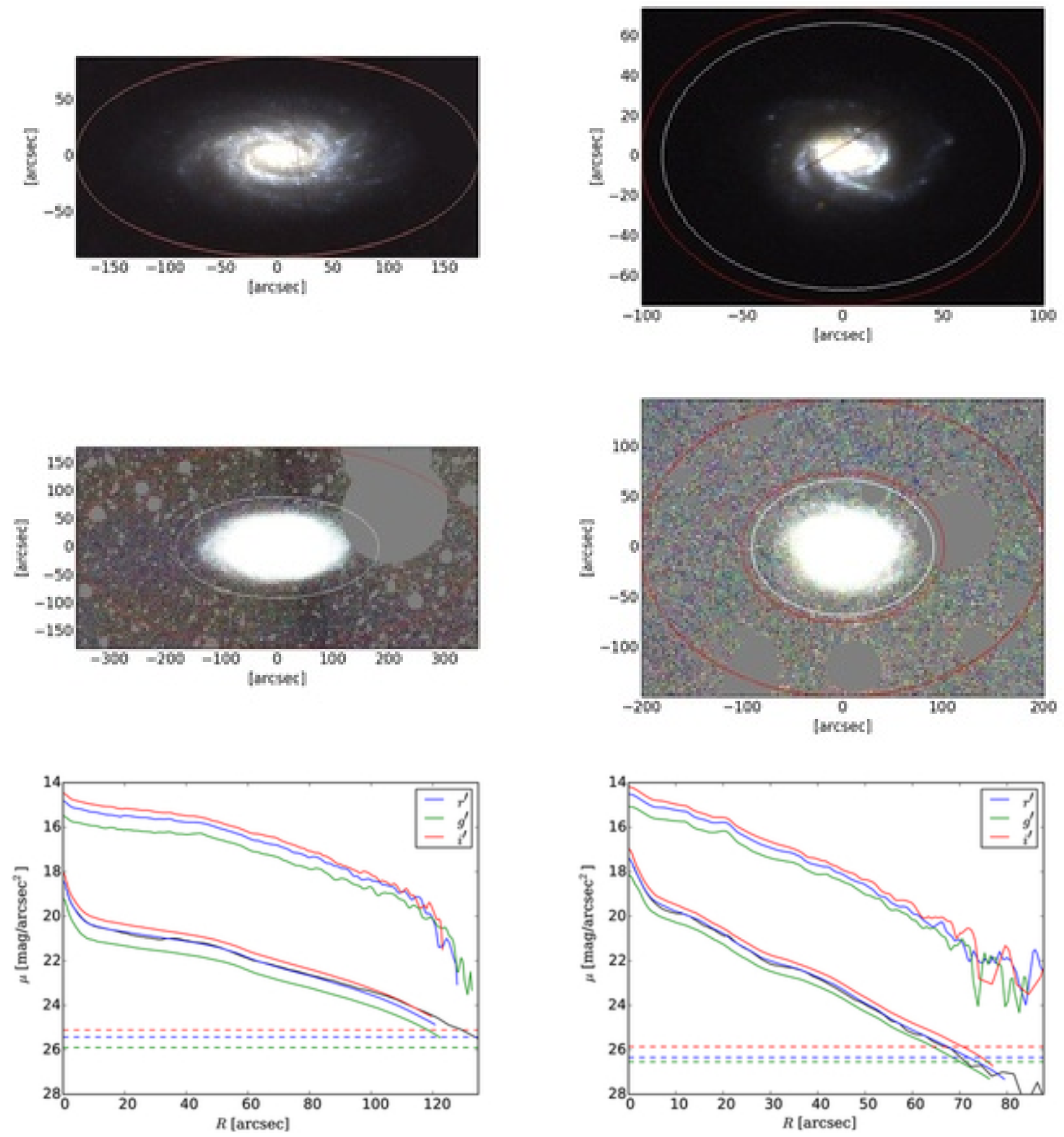

Figure A24. NGC 3756: PAS is not using the top left quadrants.

Figure A25. NGC 3888: PAS is using all quadrants. 

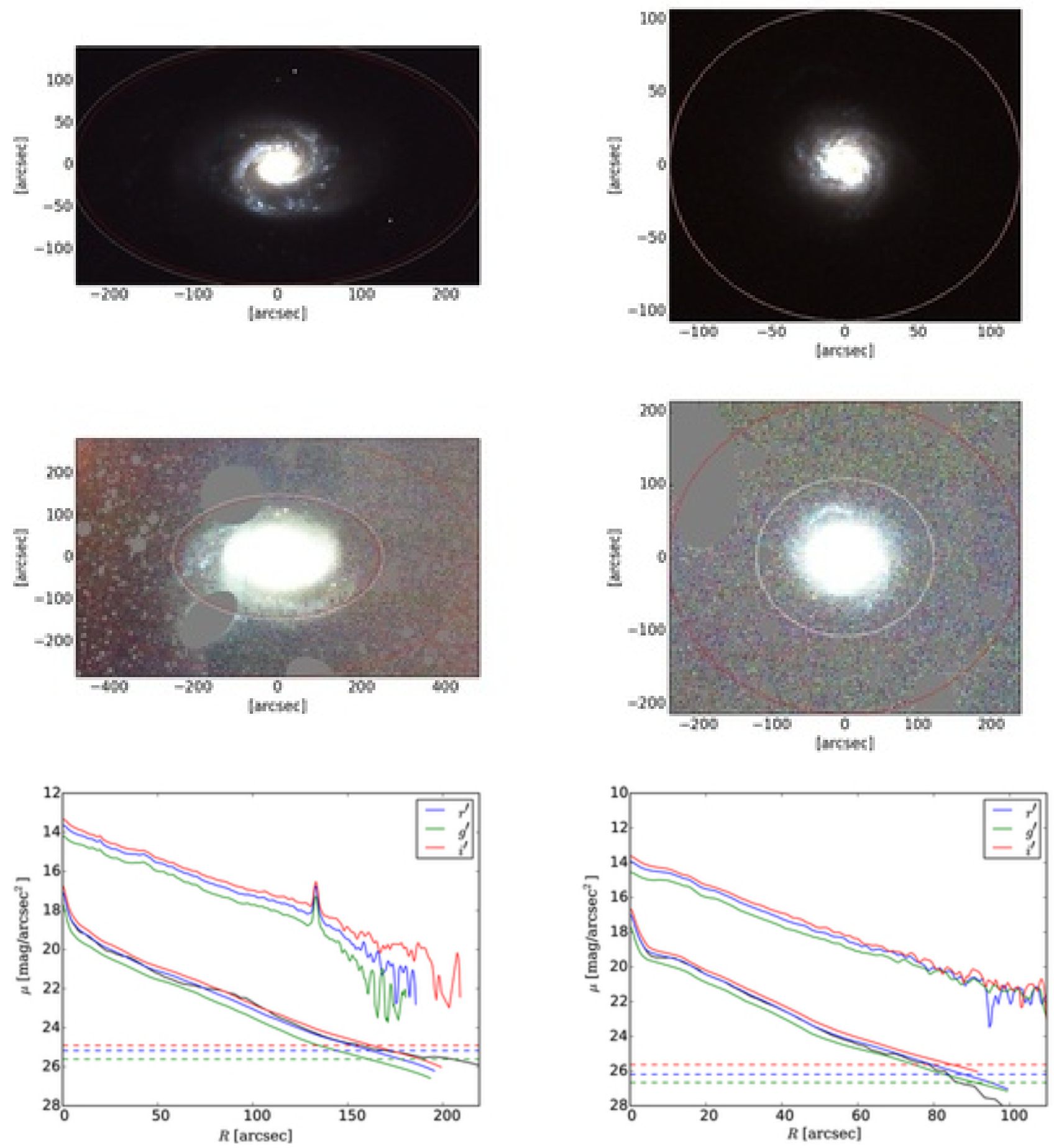

Figure A26. NGC 3893: PAS is using all quadrants.

Figure A27. NGC 3982: PAS is using all quadrants. 

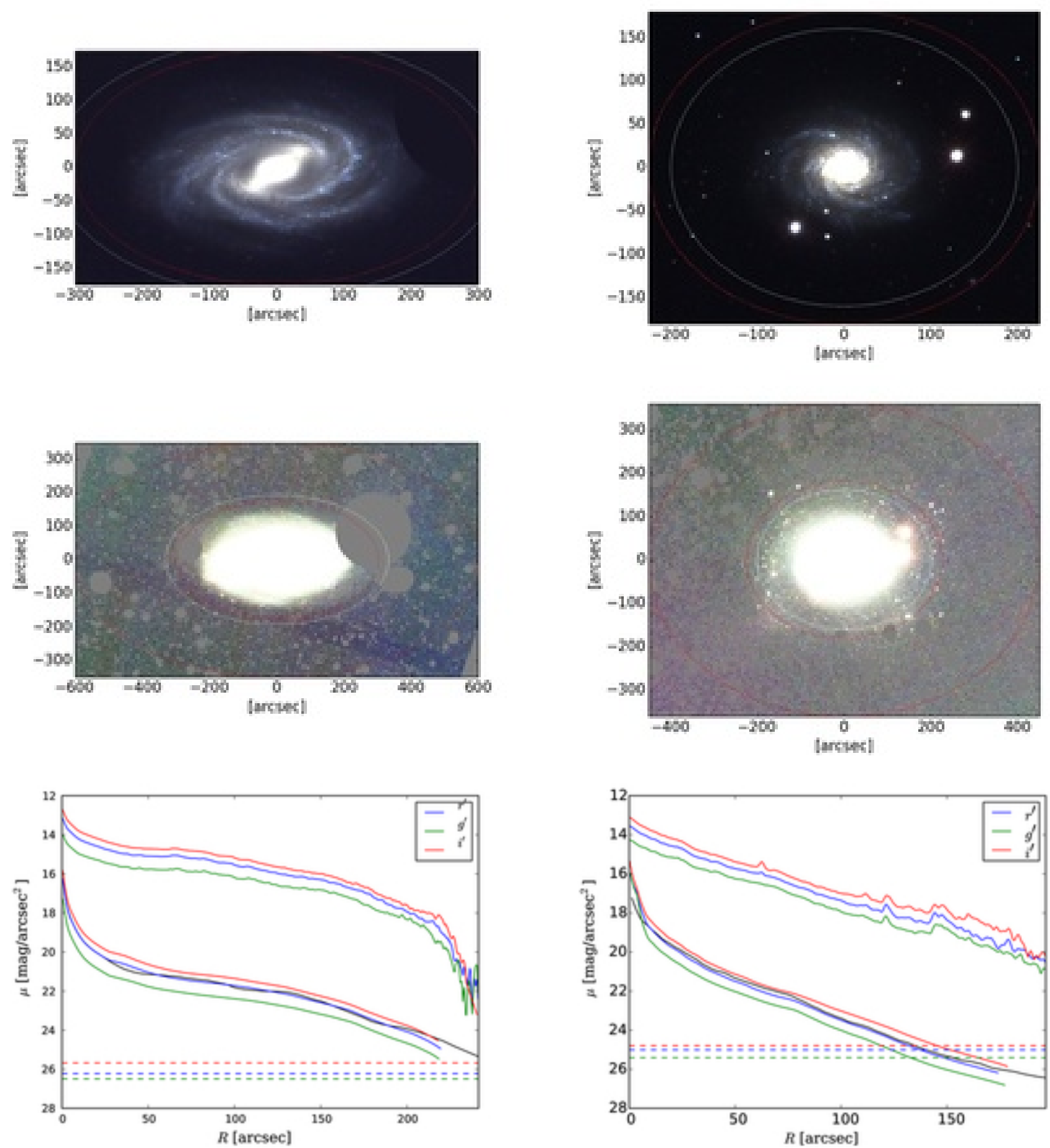

Figure A28. NGC 3992: PAS is using all quadrants.

Figure A29. NGC 4030: PAS is using all quadrants. 

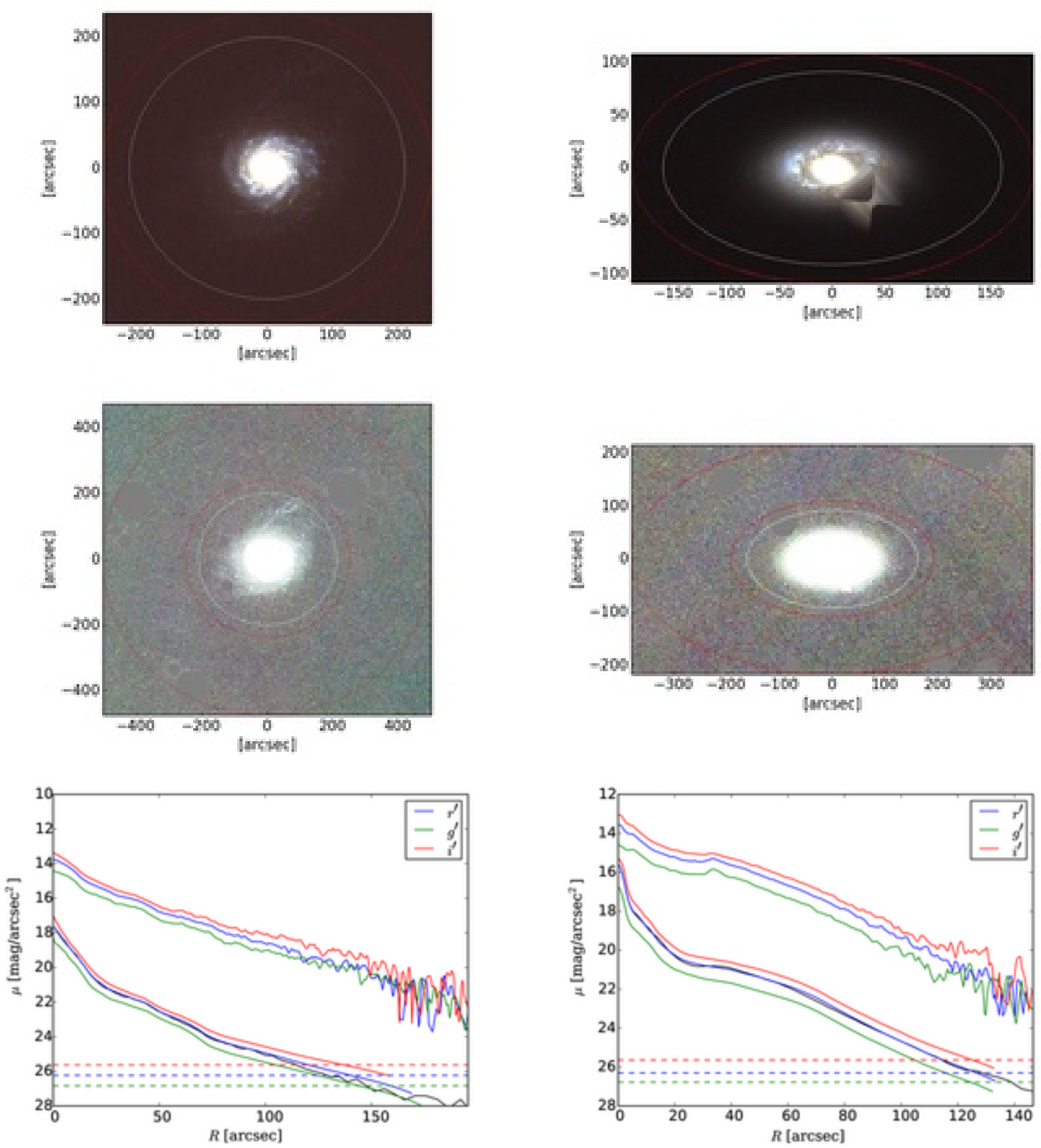

Figure A30. NGC 4041: PAS is using all quadrants.

Figure A31. NGC 4102: PAS is using the lower left, upper left and lower right quadrants. 

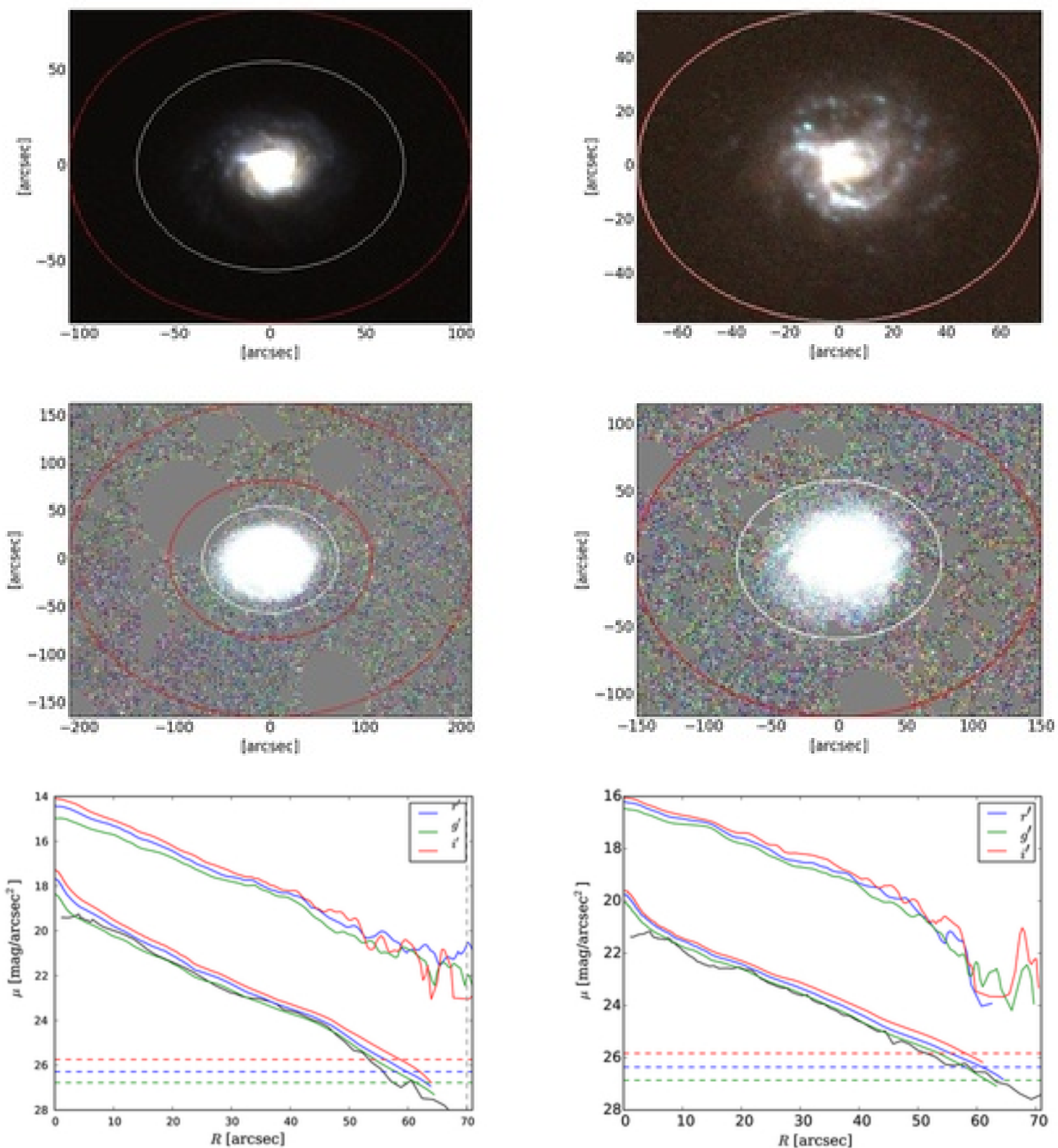

Figure A32. NGC 4108: PAS is using the upper left, lower right and upper right quadrants.

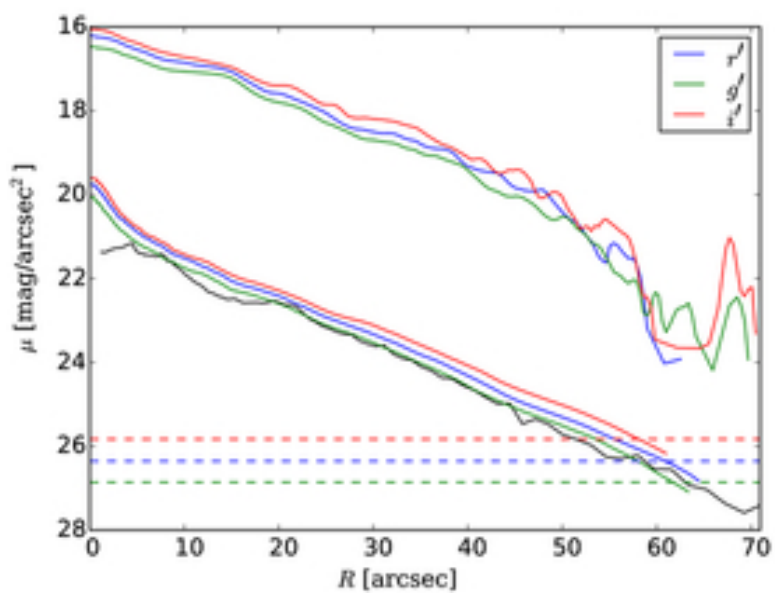

Figure A33. NGC 4108B: PAS is using all quadrants. 

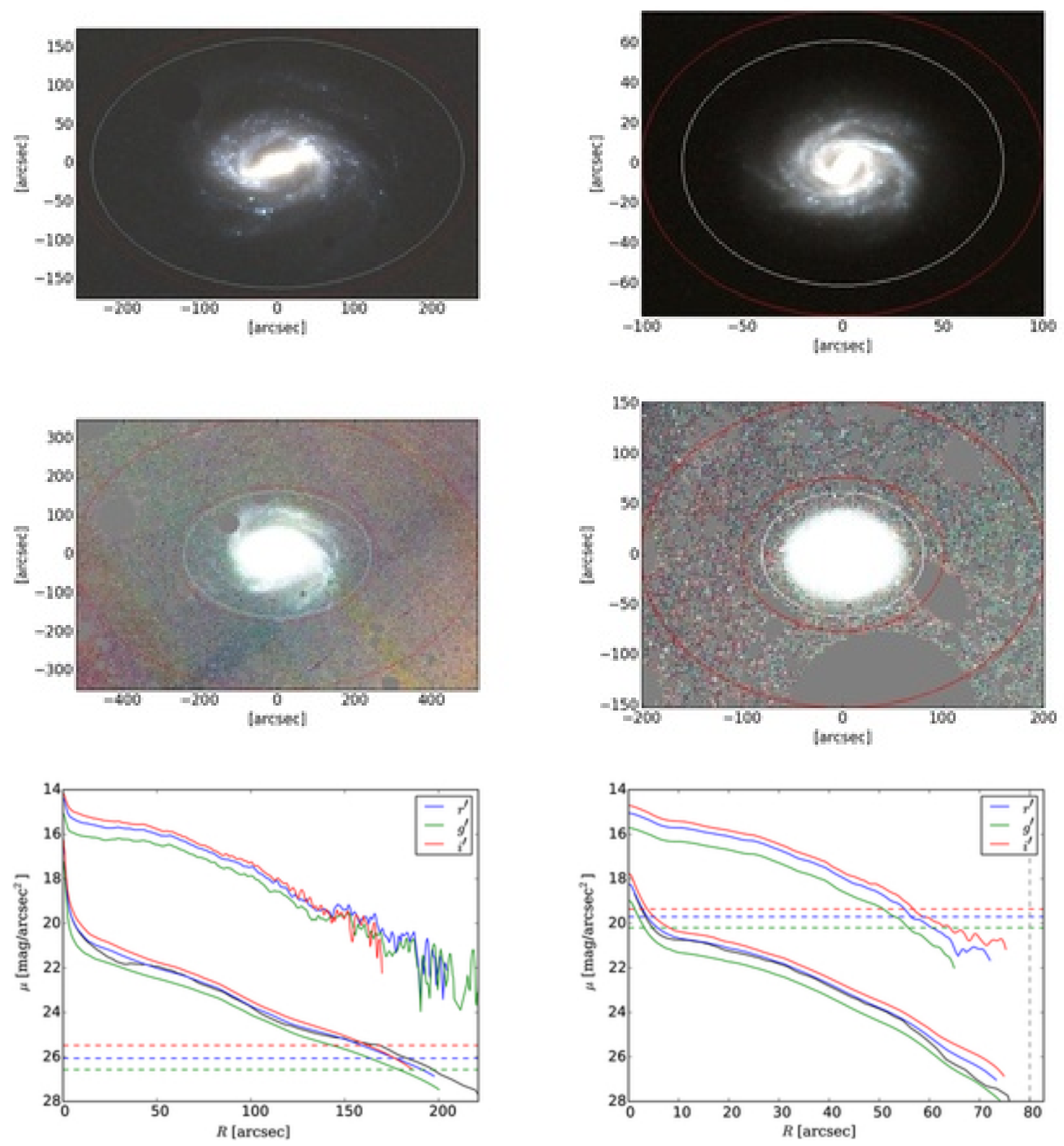

Figure A34. NGC 4123: PAS is using all quadrants.

Figure A35. NGC 4210: PAS is using all quadrants. 

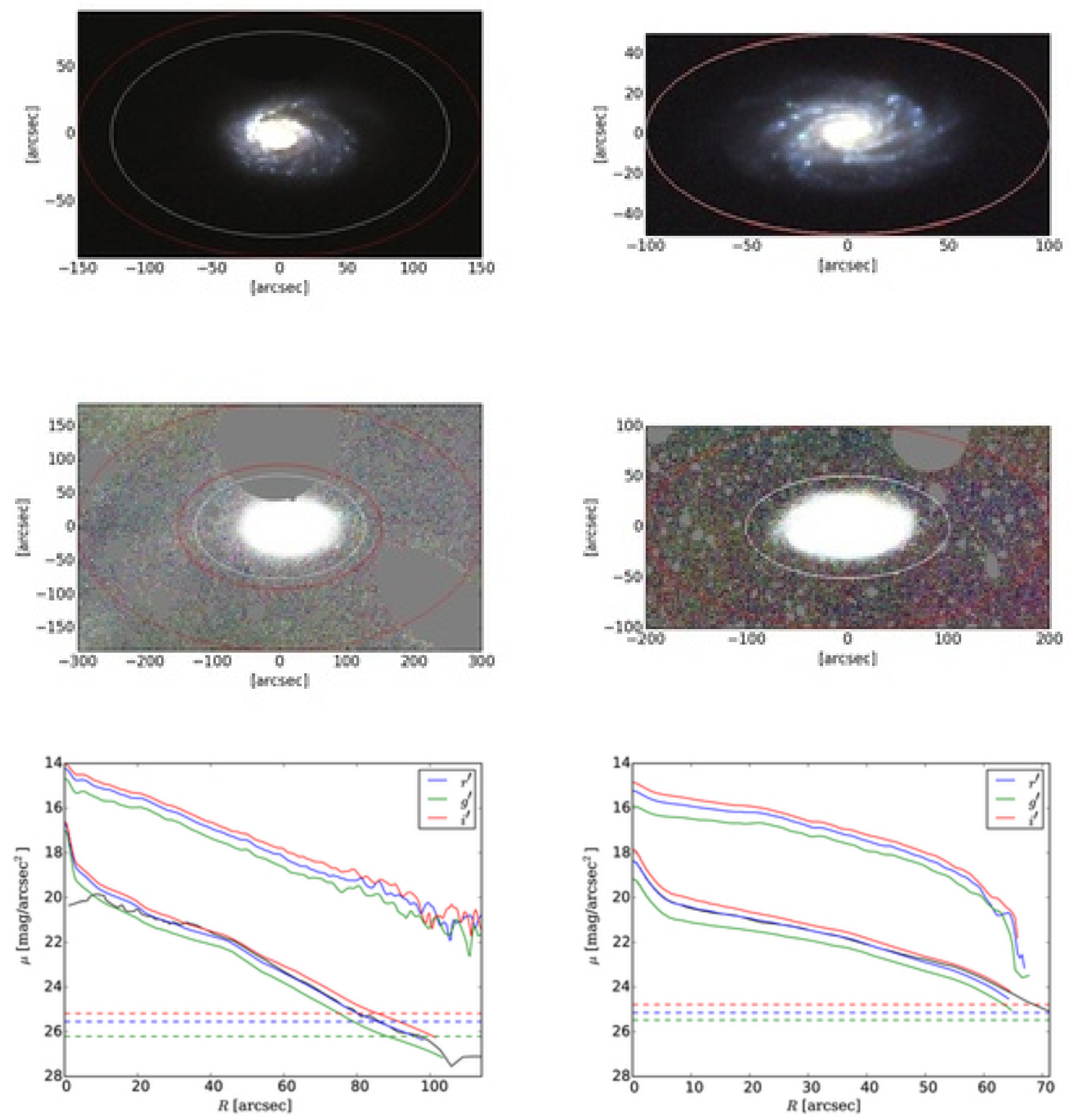

Figure A36. NGC 4273: PAS is using all quadrants.

Figure A37. NGC 4480: PAS is using all quadrants. 

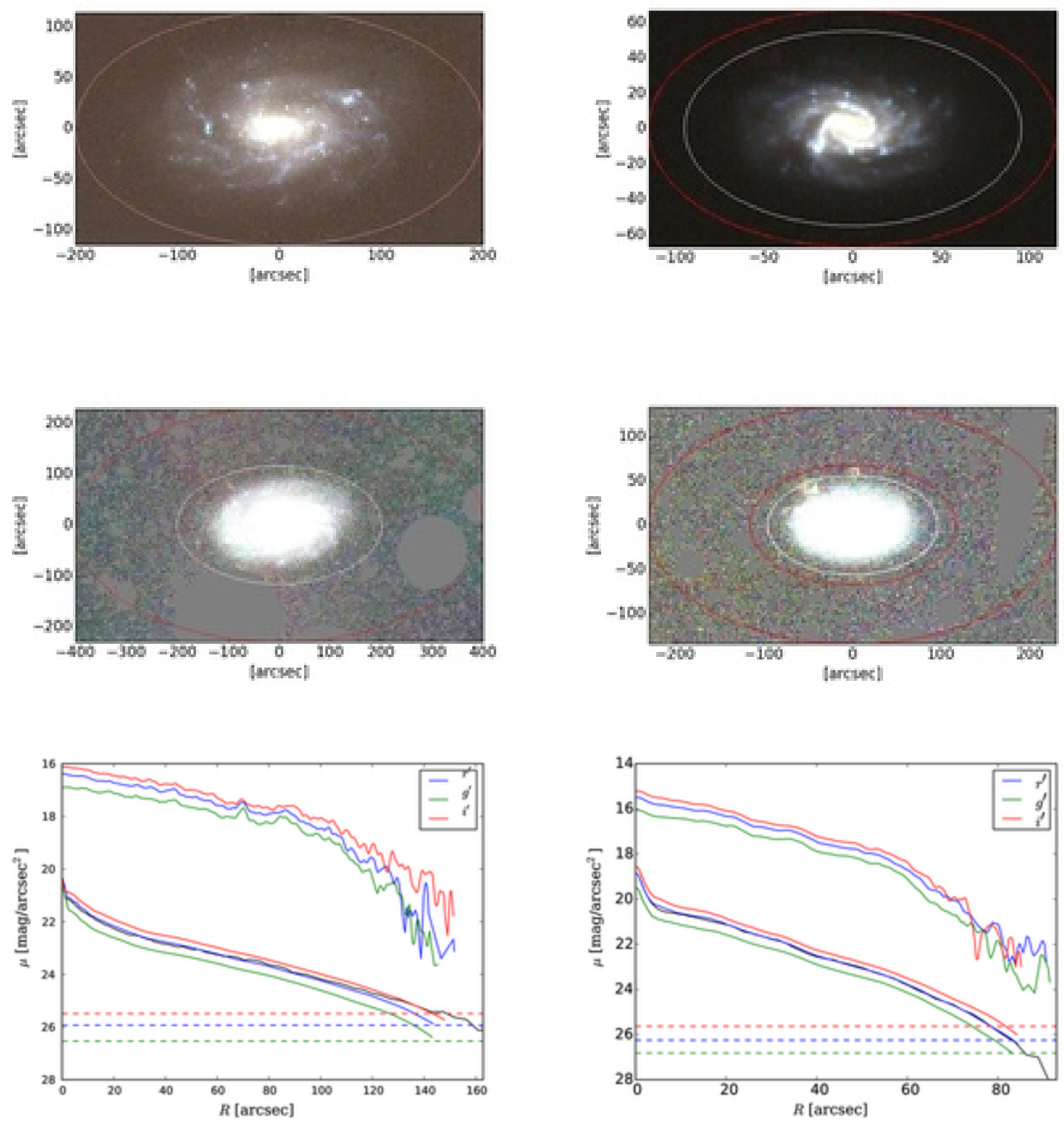

Figure A38. NGC 4517A: PAS is using all quadrants.

Figure A39. NGC 4545: PAS is using all quadrants. 

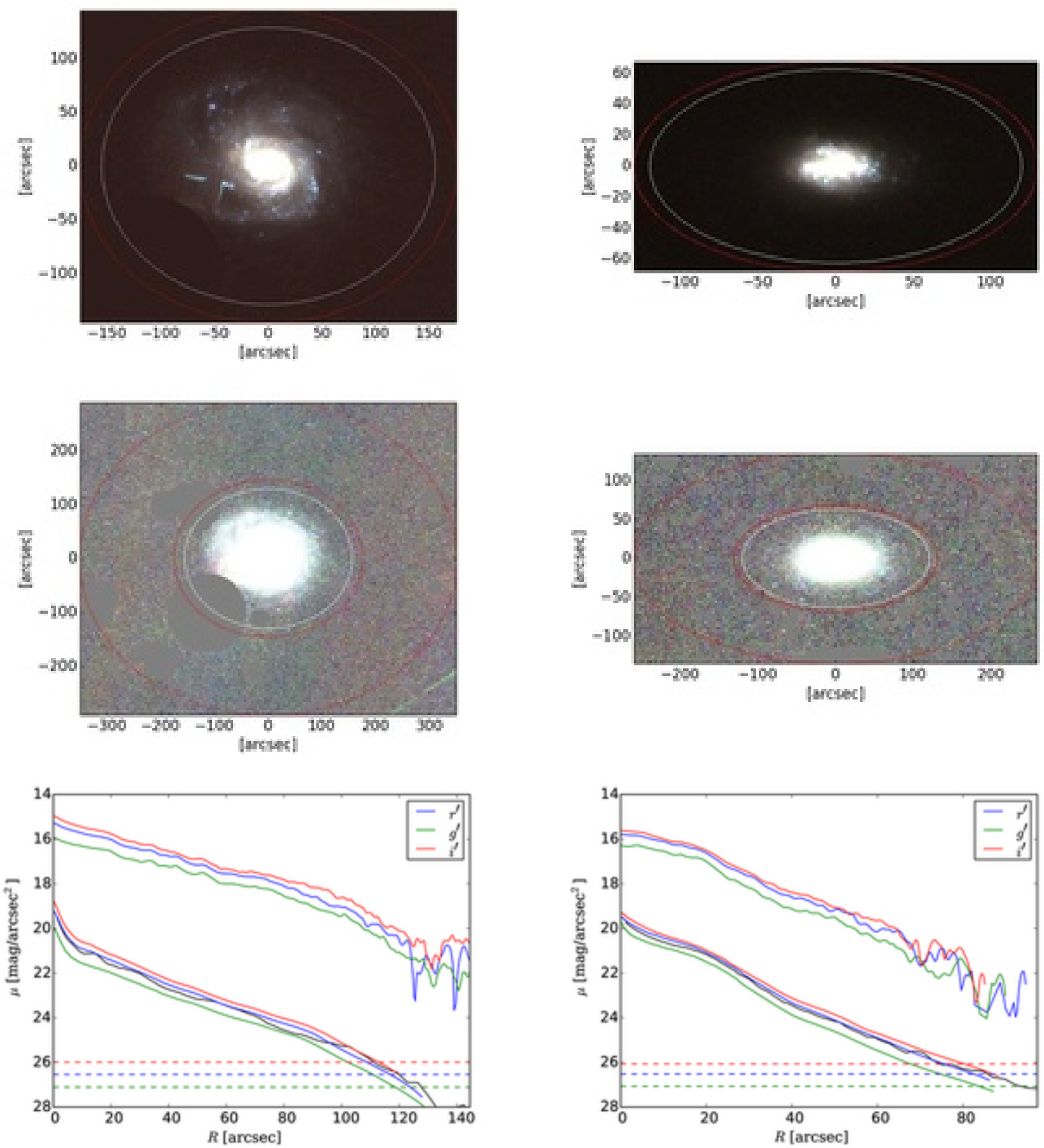

Figure A40. NGC 4653: PAS is using the PAS is using the lower right and upper right quadrants.

Figure A41. NGC 4668: PAS is using all quadrants 

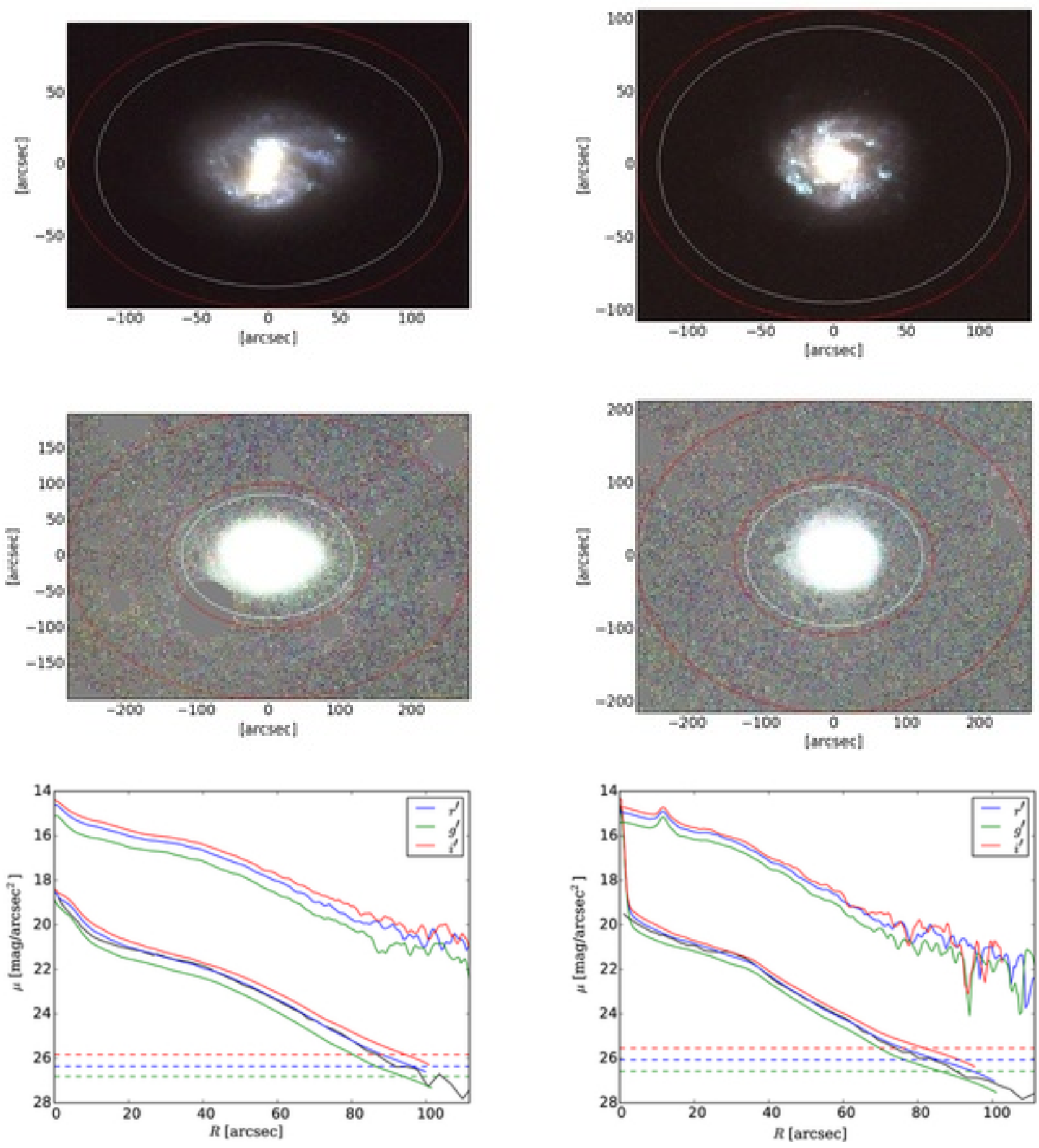

Figure A42. NGC 4904: PAS is using all quadrants.

Figure A43. NGC 5147: PAS is using all quadrants. 

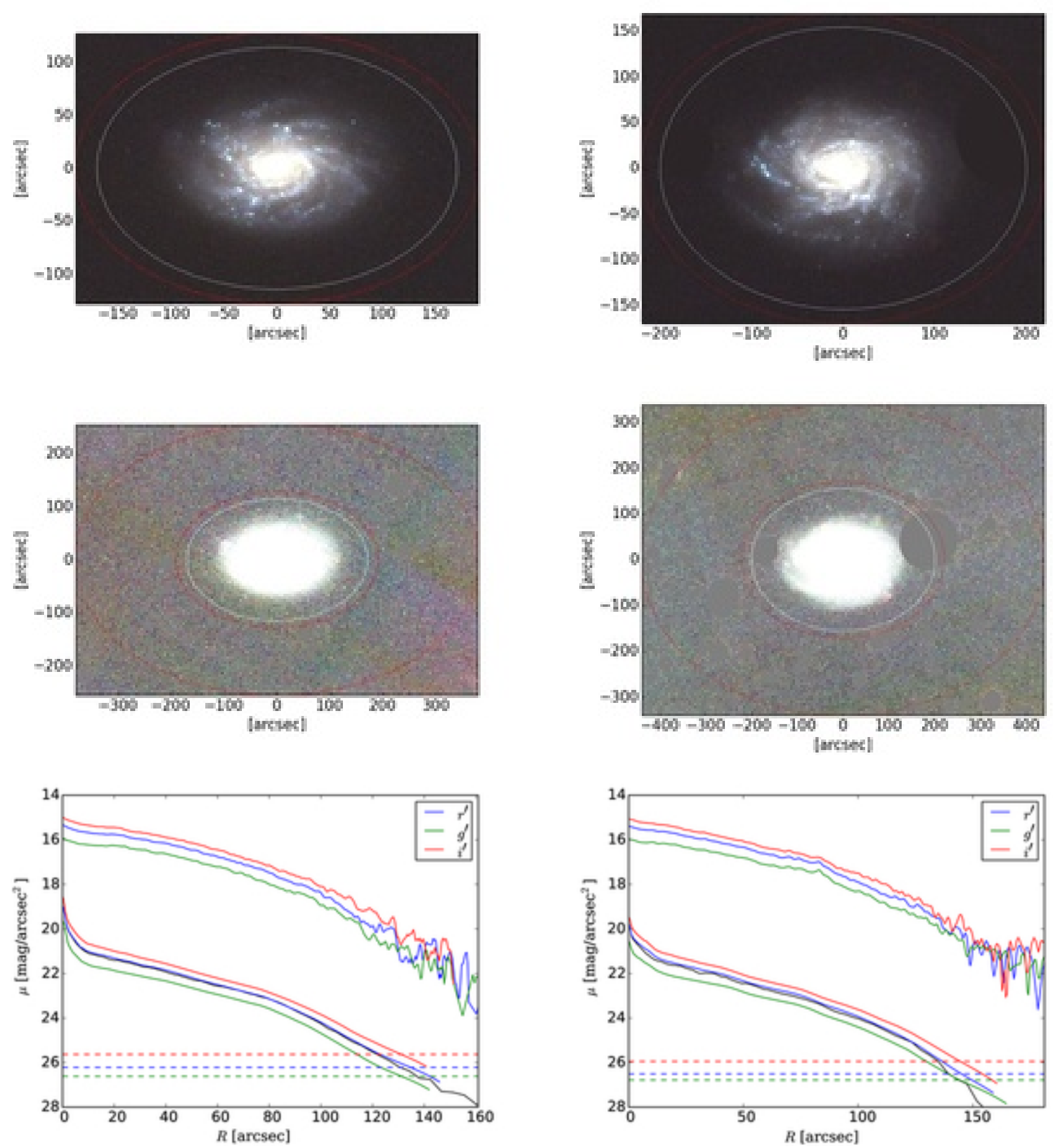

Figure A44. NGC 5300: PAS is using all quadrants.

Figure A45. NGC 5334: PAS is using the PAS is using the upper left and upper right quadrants. 

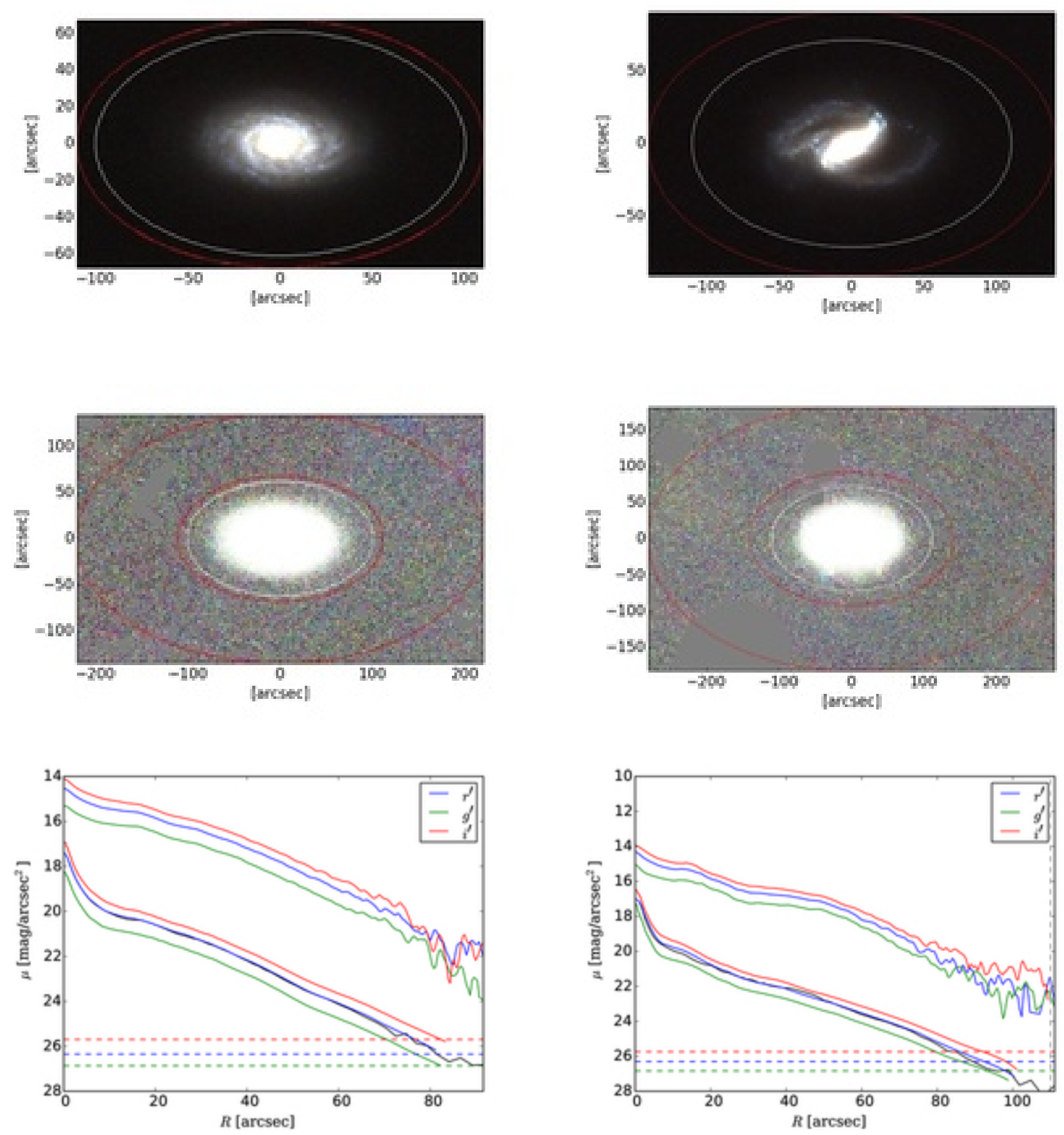

Figure A46. NGC 5376: PAS is using all quadrants.

Figure A47. NGC 5430: PAS is using all quadrants. 

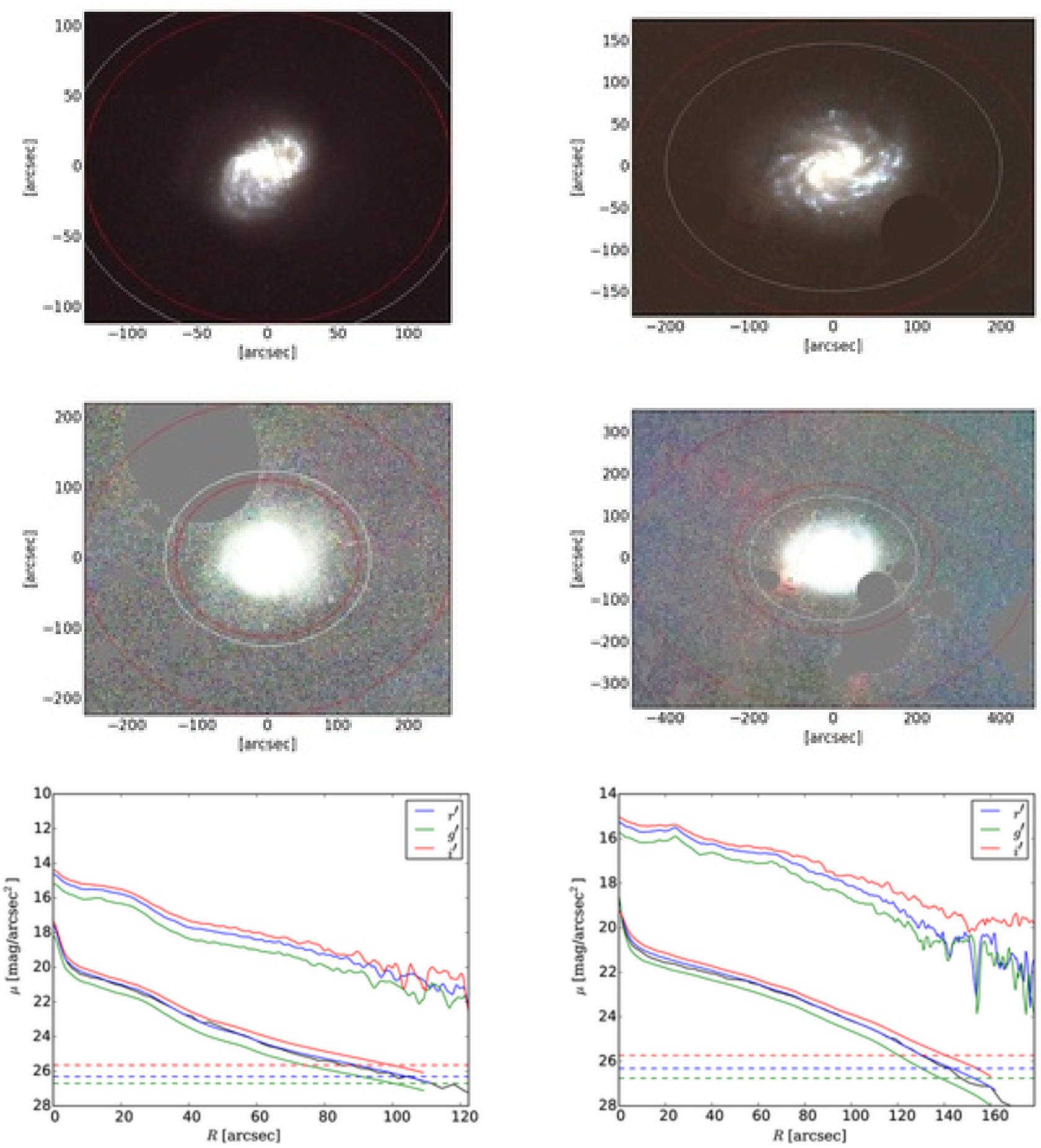

Figure A48. NGC 5480: PAS is using all quadrants.

Figure A49. NGC 5584: PAS is using all quadrants. 

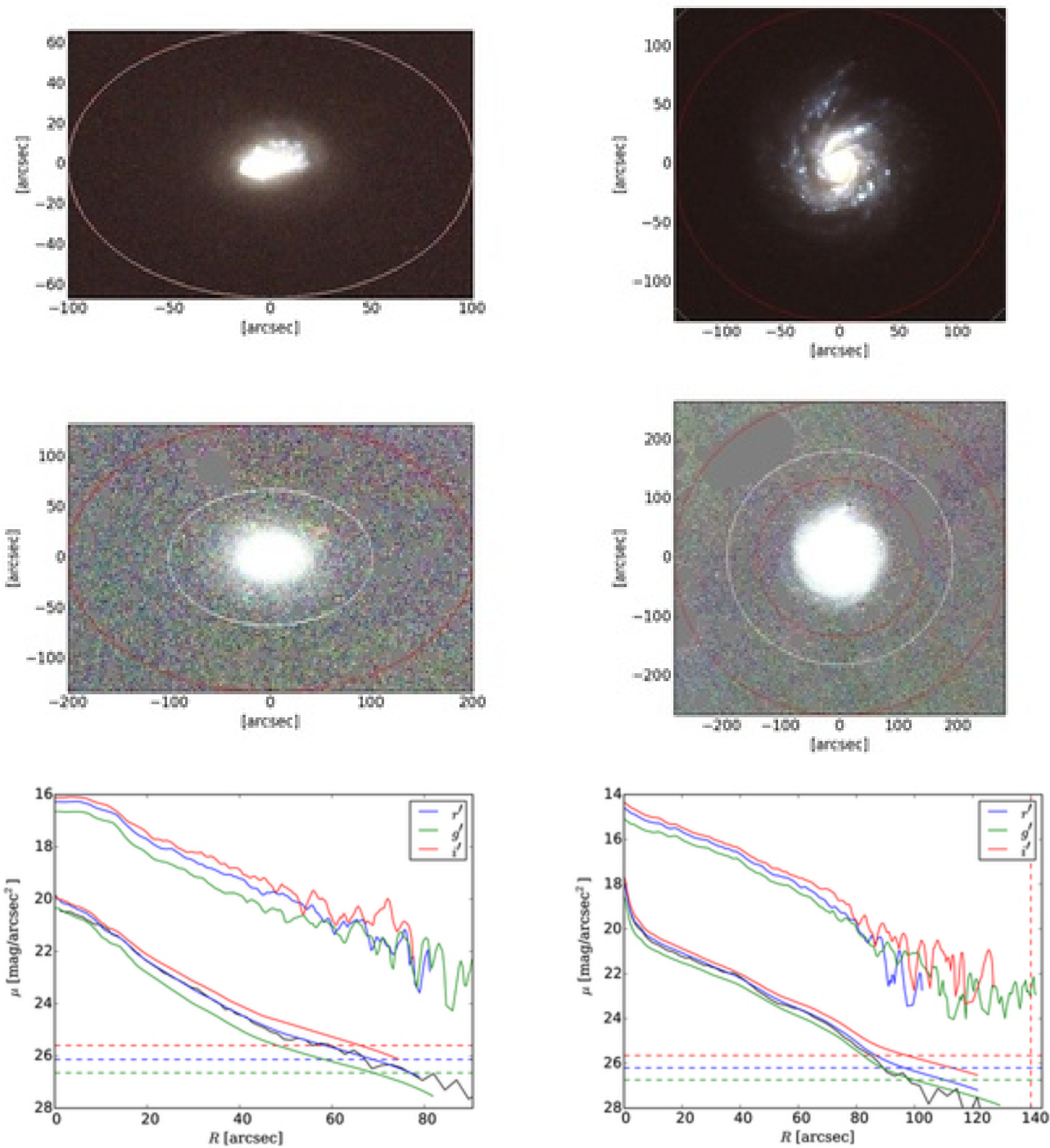

Figure A50. NGC 5624: PAS is using all quadrants.

Figure A51. NGC 5660: PAS is using all quadrants. 

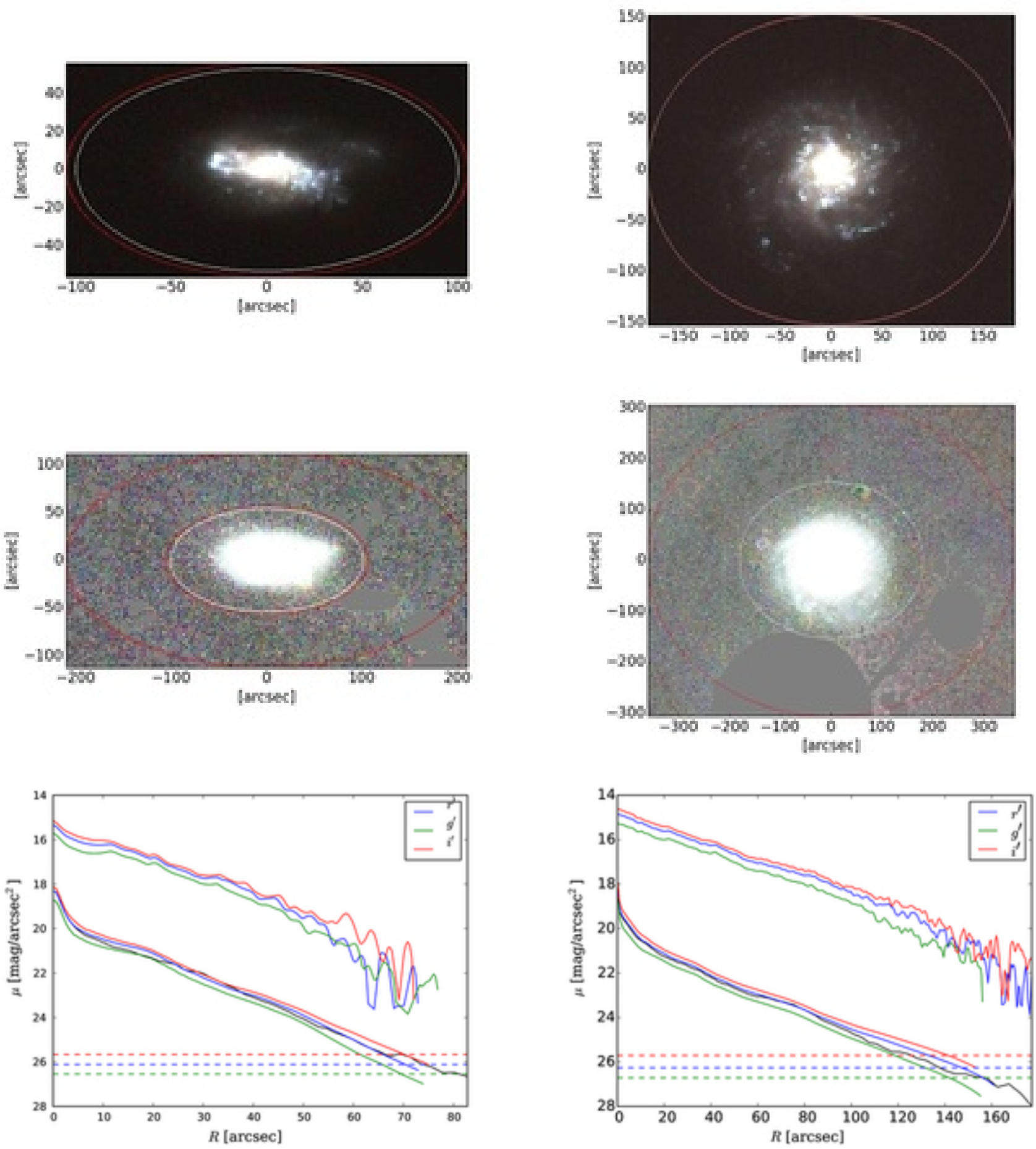

Figure A52. NGC 5667: PAS is using the upper left, lower right and upper right quadrants.

Figure A53. NGC 5668: PAS is using all quadrants. 

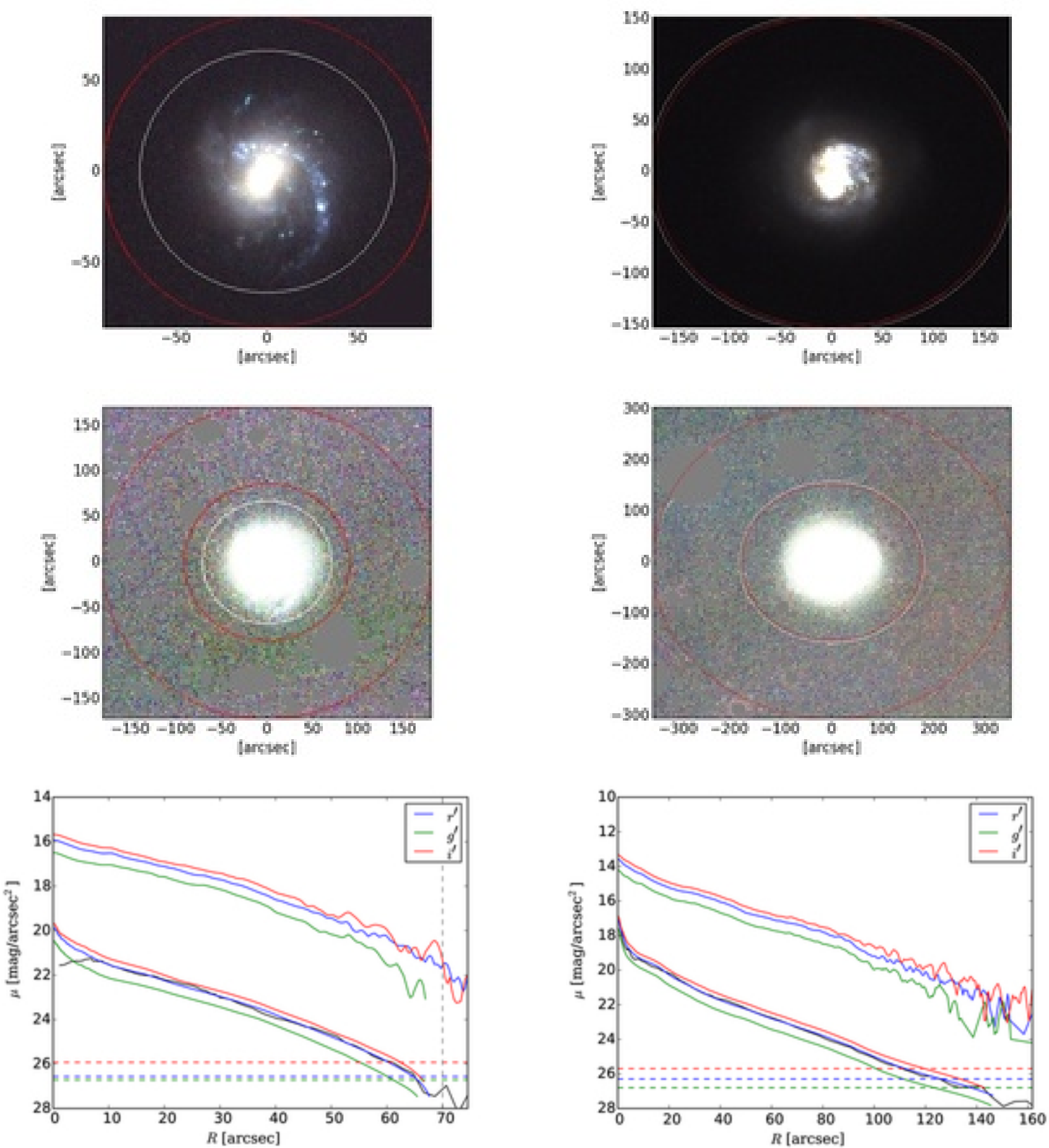

Figure A54. NGC 5693: PAS is using all quadrants.

Figure A55. NGC 5713: PAS is using all quadrants. 

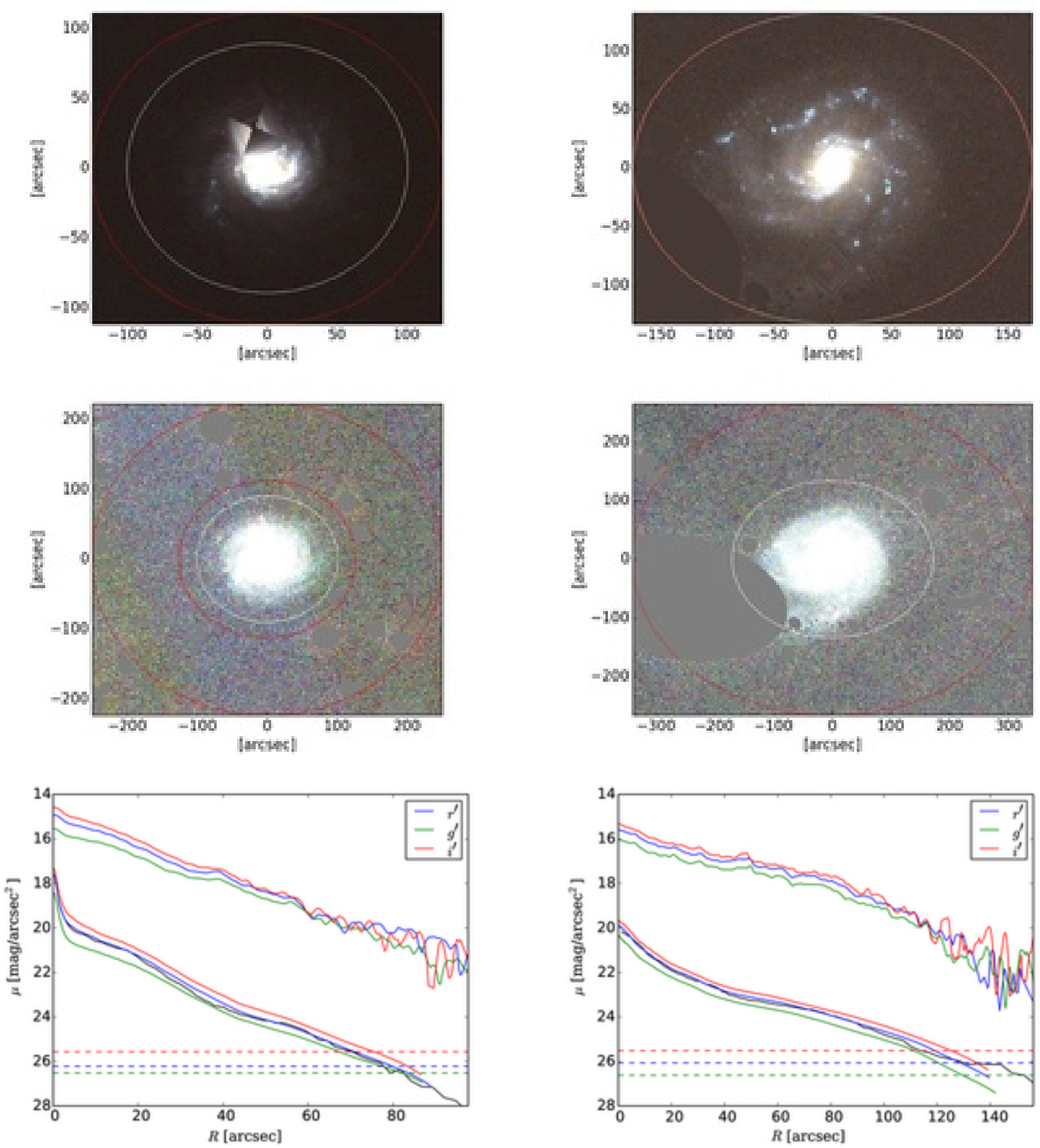

Figure A56. NGC 5768: PAS is using all quadrants.

Figure A57. NGC 5774: PAS is using the lower left, lower right and upper right quadrants. 

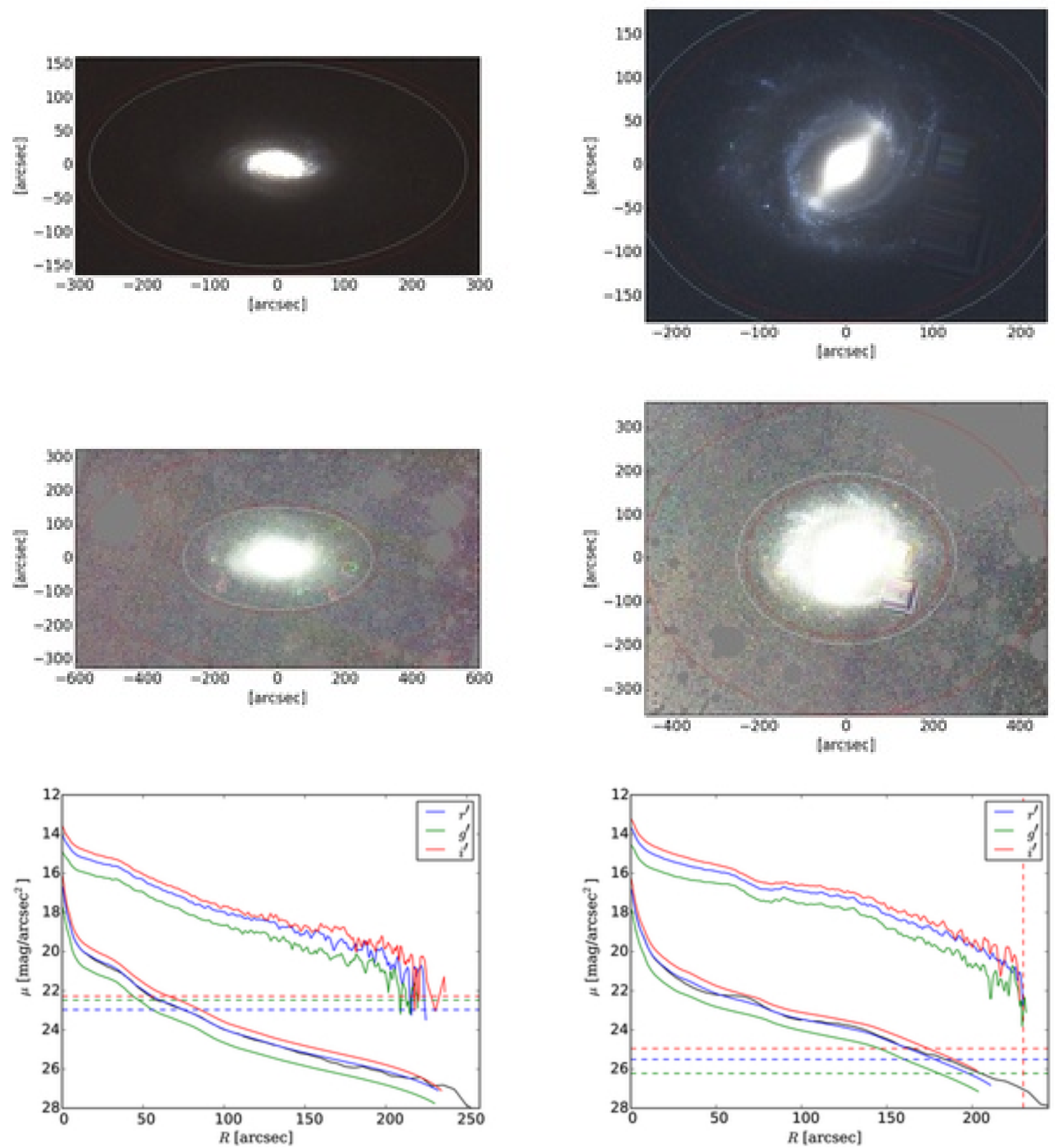

Figure A58. NGC 5806: PAS is using all quadrants.

Figure A59. NGC 5850: PAS is using all quadrants. 

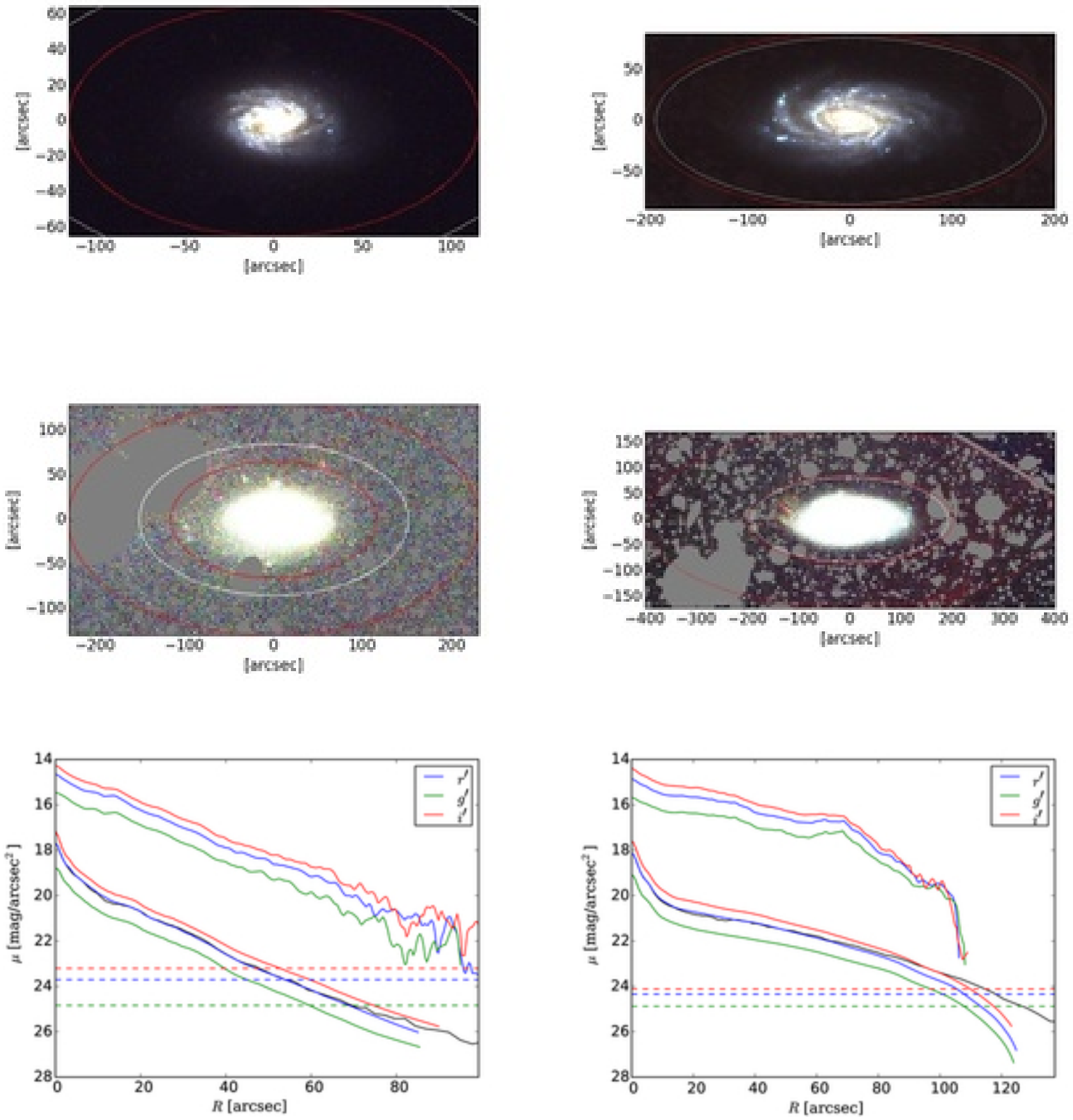

Figure A60. NGC 5937: PAS is not using the top left quadrant.

Figure A61. NGC 6070: PAS is using all quadrants. 

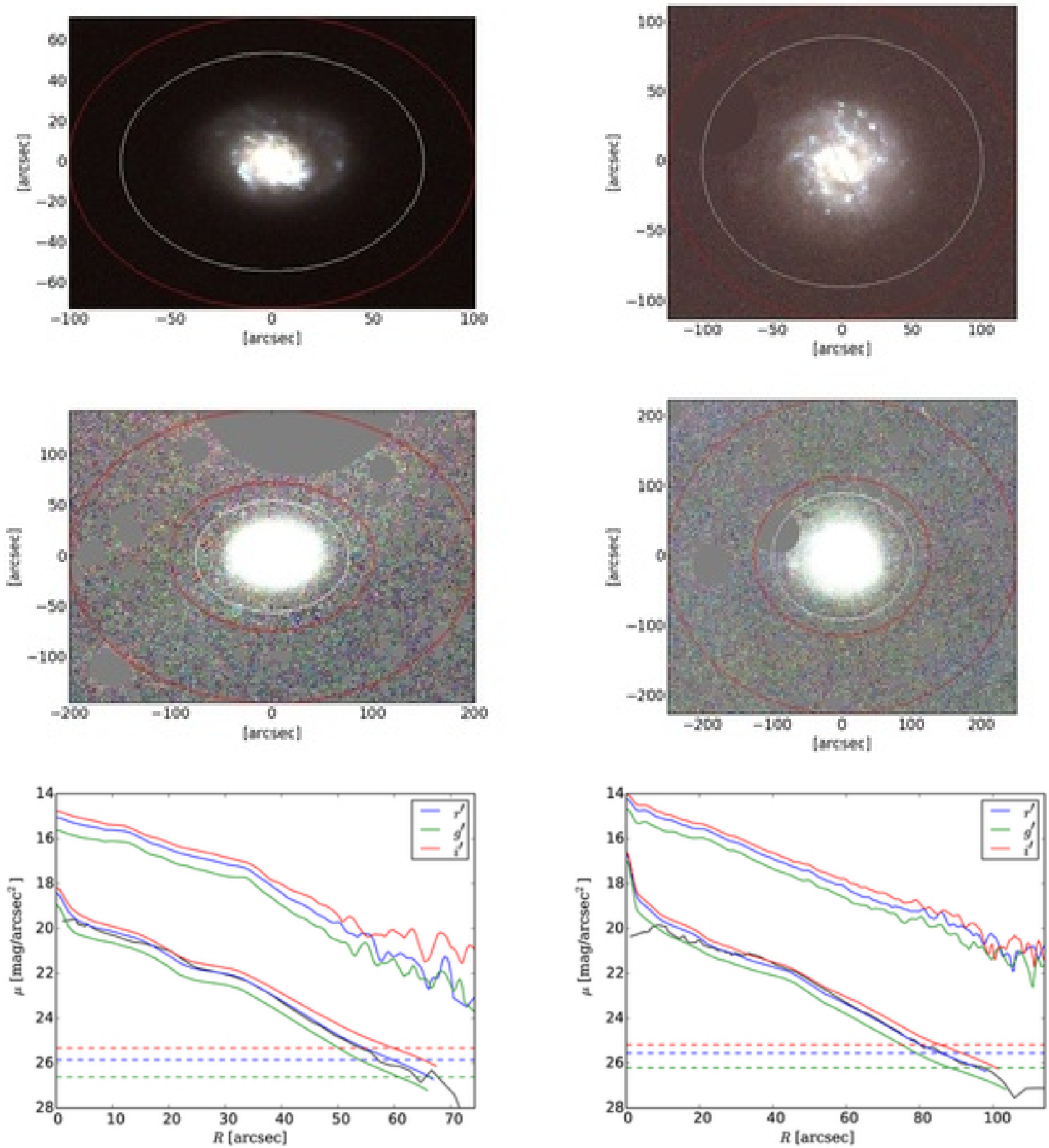

Figure A62. NGC 6155: PAS is using all quadrants.

Figure A63. NGC 7437: PAS is using the upper left, lower right and upper right quadrants. 

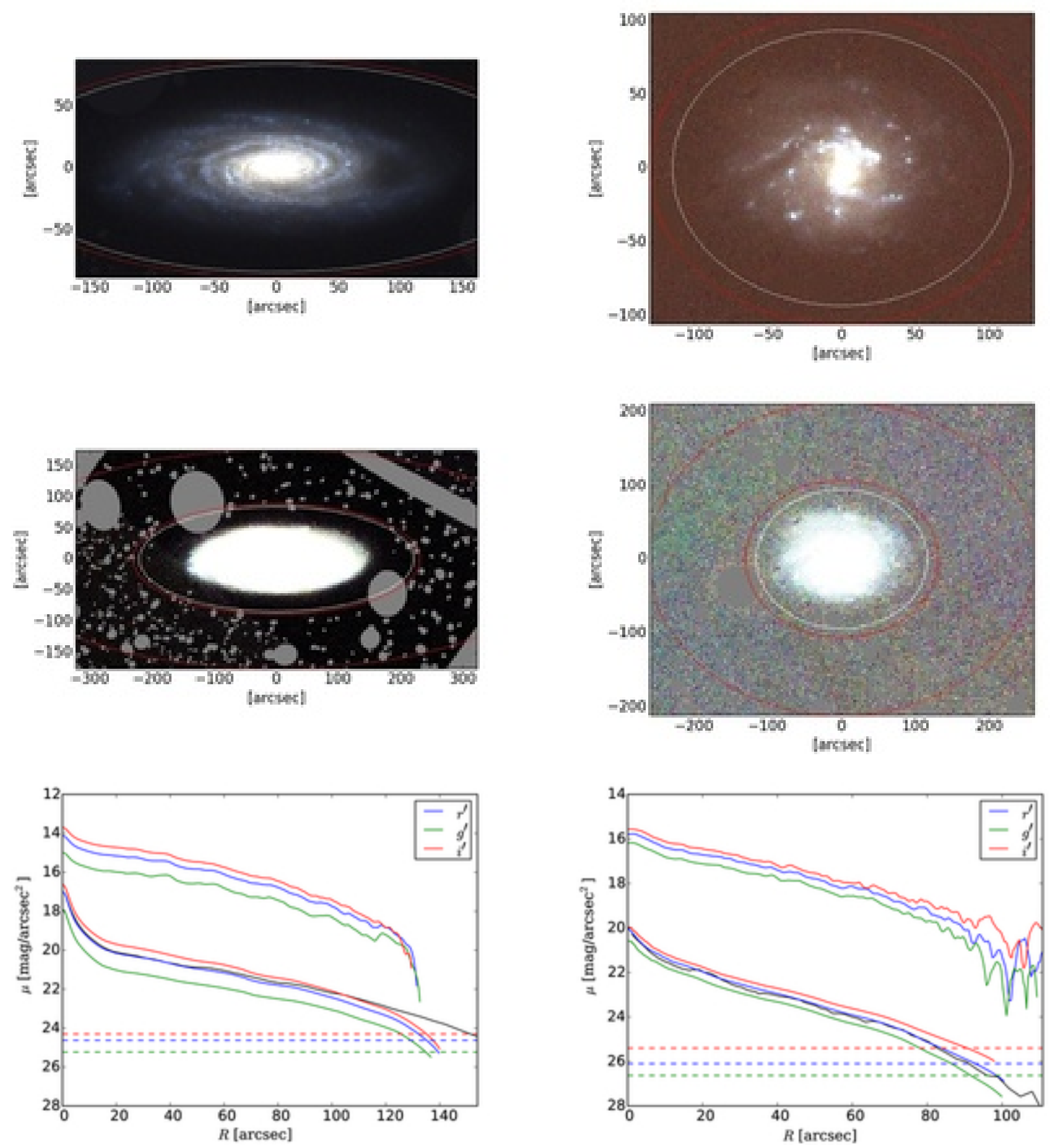

Figure A64. NGC 7606: PAS is using all quadrants.

Figure A65. PGC 006667: PAS is using all quadrants. 

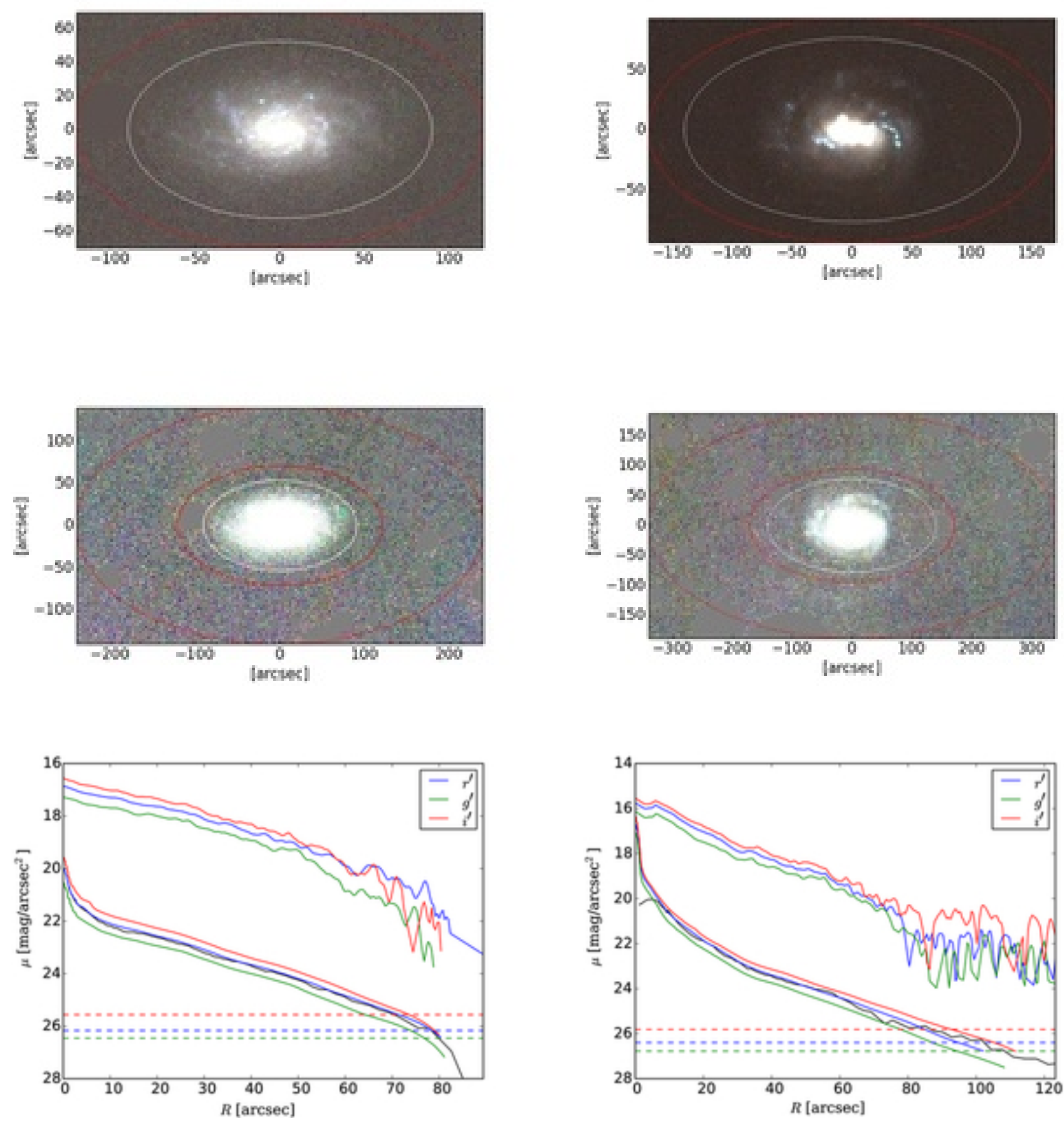

Figure A66. UGC 02081: PAS is using all quadrants.

Figure A67. UGC 04393: PAS is using all quadrants. 

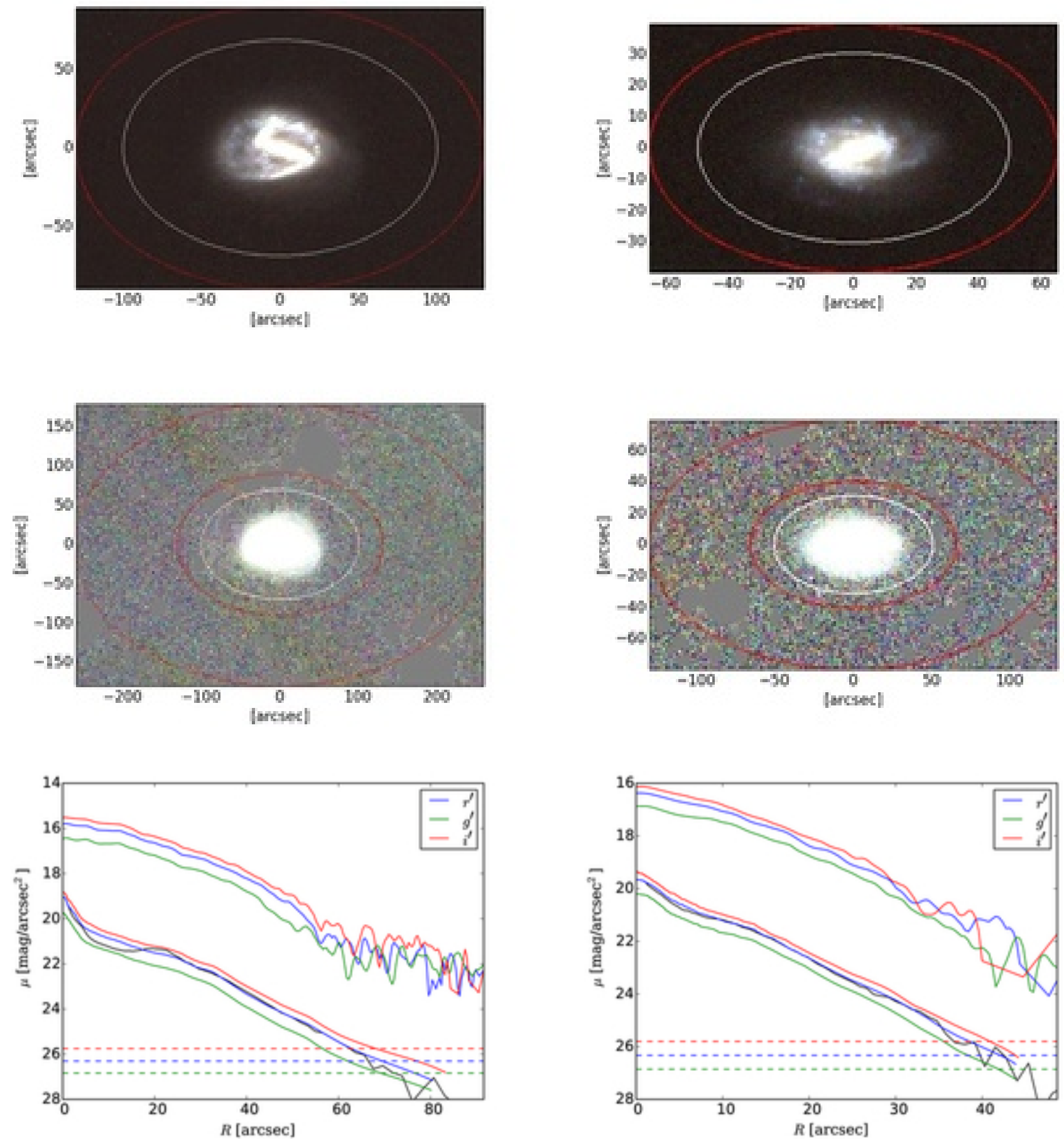

Figure A68. UGC 06309: PAS is using all quadrants.

Figure A69. UGC 06518: PAS is using all quadrants. 

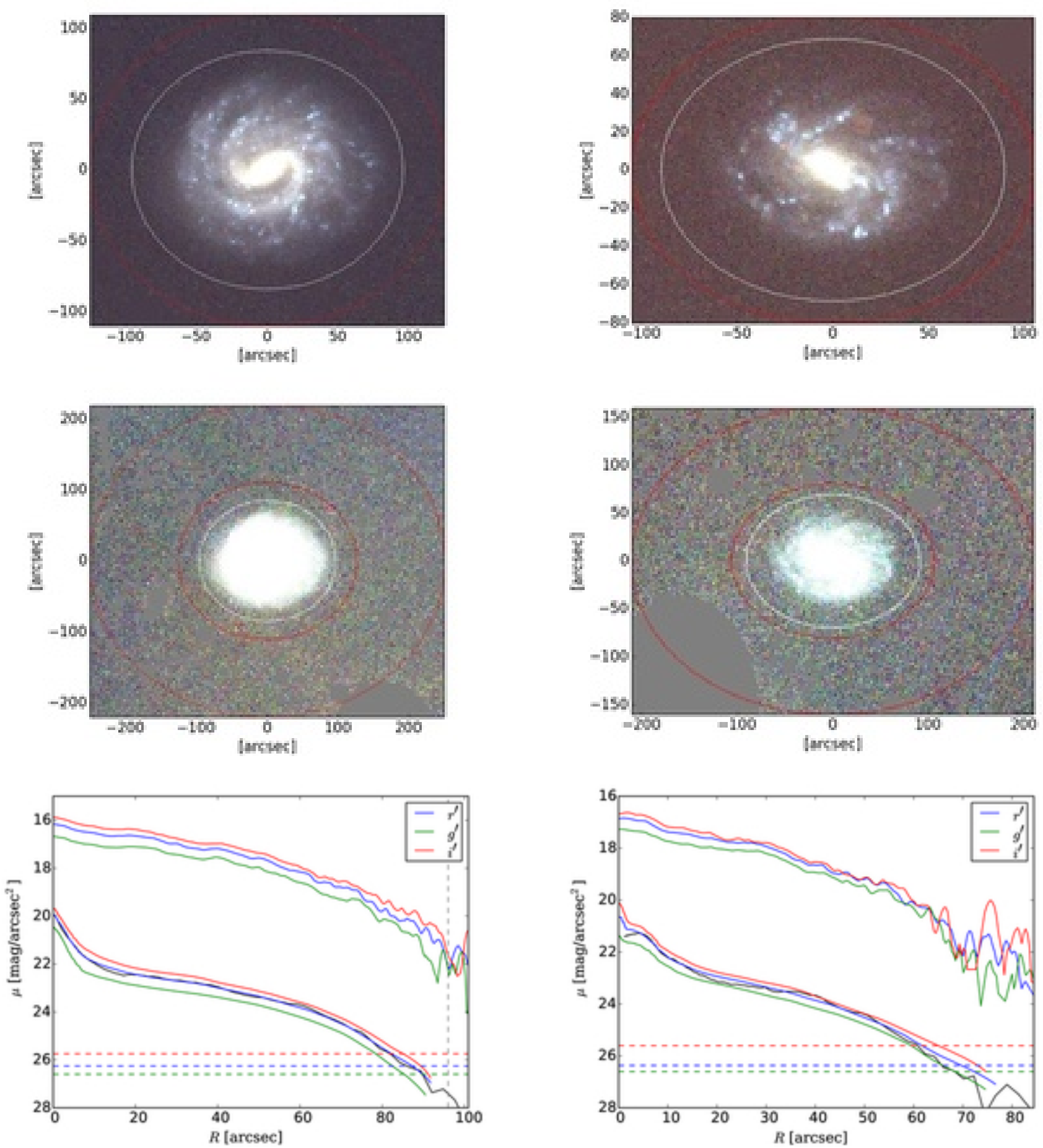

Figure A70. UGC 06903: PAS is using all quadrants.

Figure A71. UGC 07700: PAS is using all quadrants. 

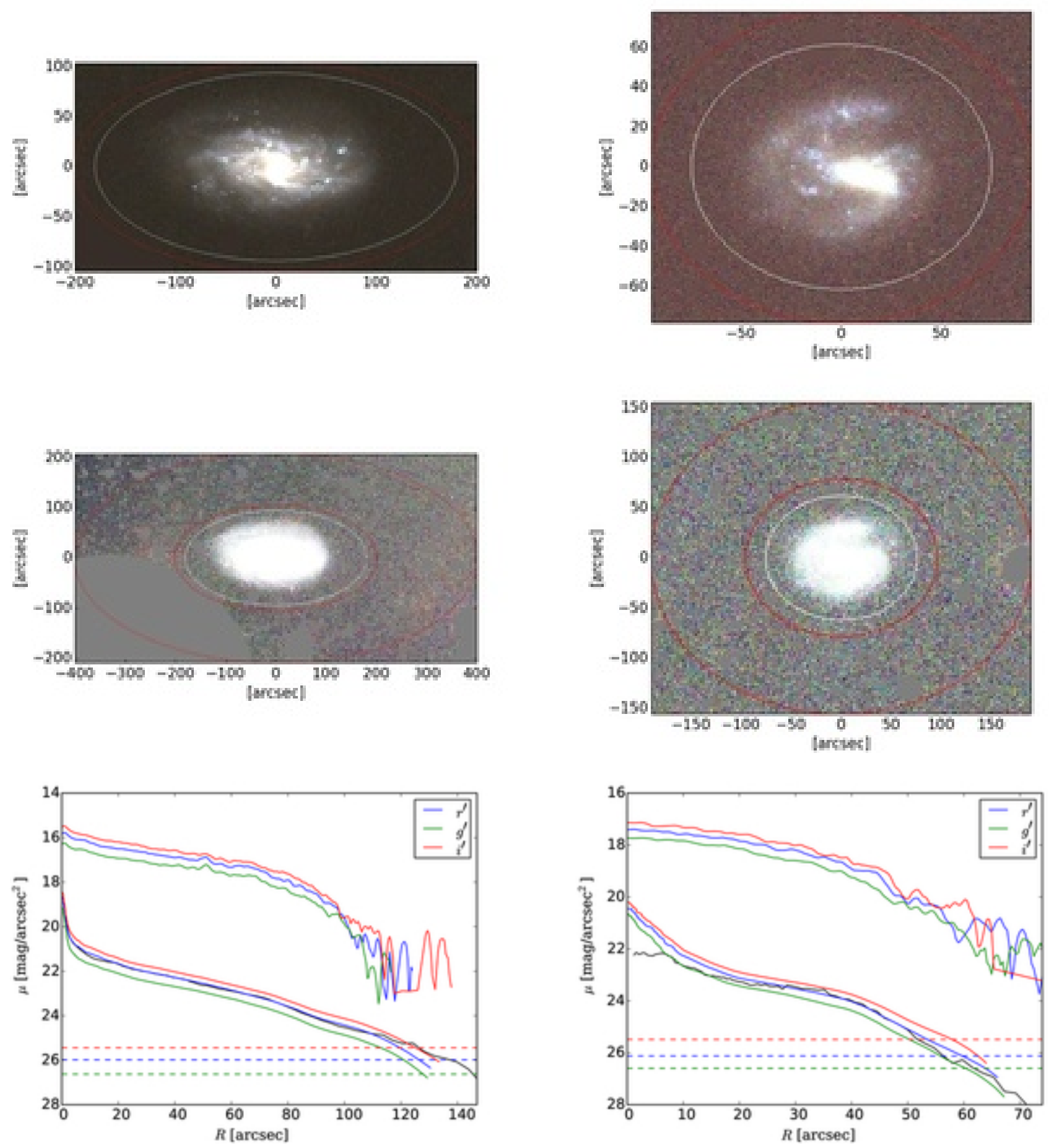

Figure A72. UGC 08041: PAS is not using lower left quadrant.

Figure A73. UGC 08084: PAS is using all quadrants. 

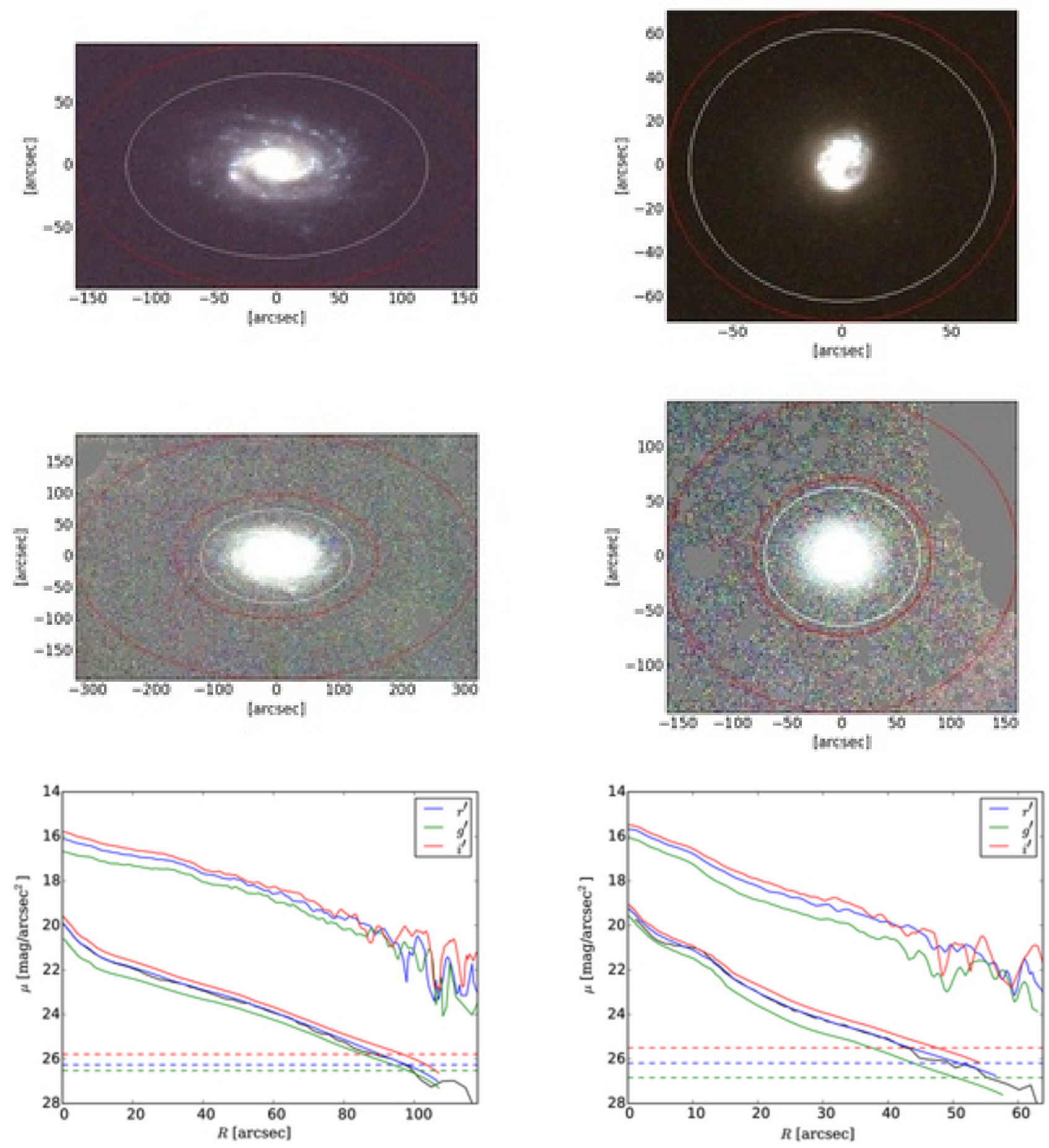

Figure A74. UGC 08658: PAS is using all quadrants.

Figure A75. UGC 09741: PAS is using all quadrants. 

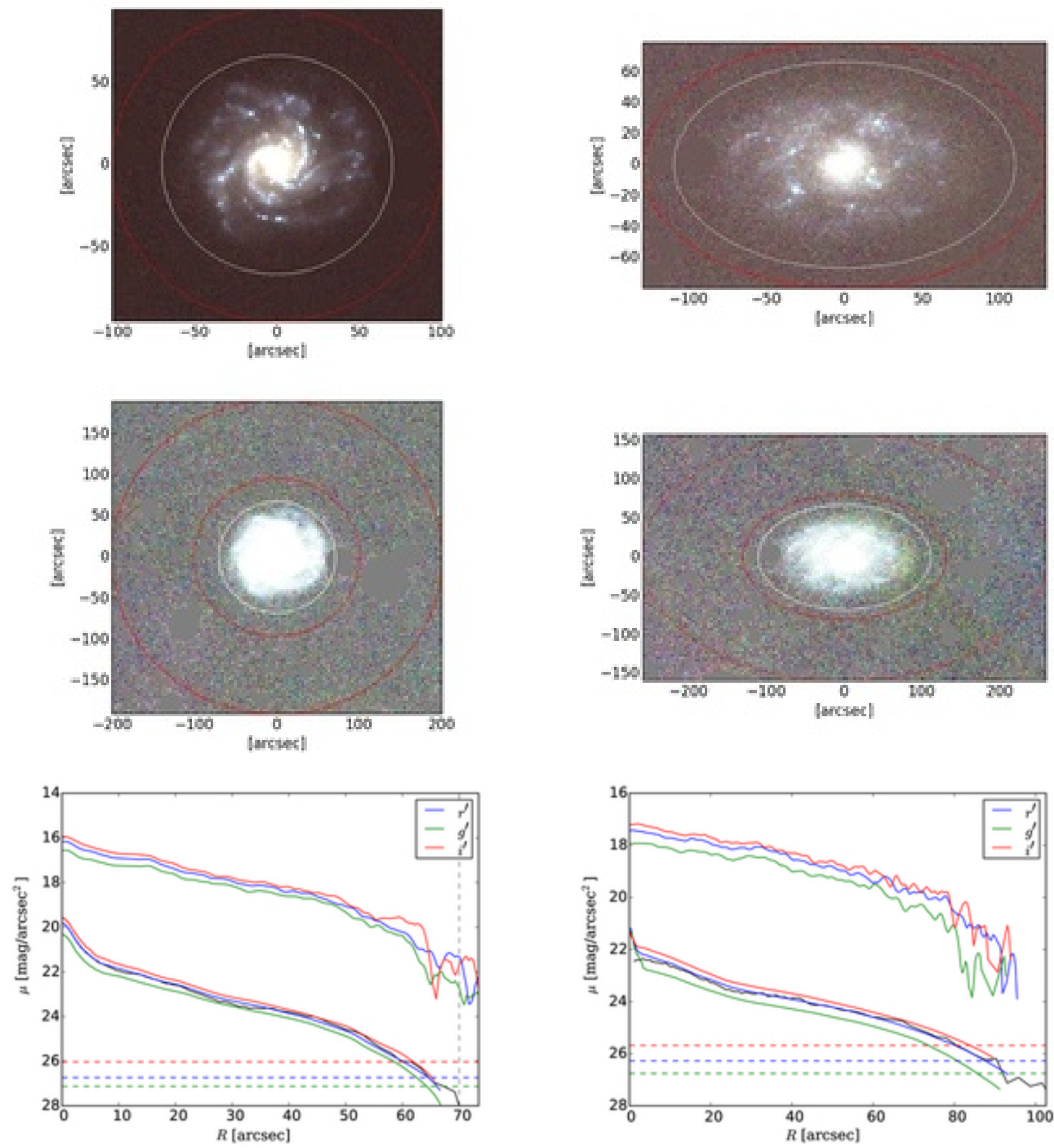

Figure A76. UGC 09837: PAS is using all quadrants.

Figure A77. UGC 12709: PAS is using all quadrants. 
This paper has been typeset from a $\mathrm{T}_{\mathrm{E}} \mathrm{X} / \mathrm{LT}_{\mathrm{E}} \mathrm{X}$ file prepared by the author. 